\title{
UNIVERSITYOF
}

FORWARD

THINKING

WESTMINSTER用

WestminsterResearch

http://www.westminster.ac.uk/westminsterresearch

\section{Branching-time logic ECTL\# and its tree-style one-pass tableau: Extending fairness expressibility of ECTL+}

\author{
Bolotov, A., Hermo, M. and Lucio, P.
}

NOTICE: this is the authors' version of a work that was accepted for publication in Theoretical Computer Science. Changes resulting from the publishing process, such as peer review, editing, corrections, structural formatting, and other quality control mechanisms may not be reflected in this document. Changes may have been made to this work since it was submitted for publication. A definitive version was subsequently published in Theoretical Computer Science, 813, pp. 428-451, 2020.

The final definitive version in Theoretical Computer Science is available online at:

https://doi.org/10.1016/j.tcs.2020.02.015.

(C) 2020. This manuscript version is made available under the CC-BY-NC-ND 4.0 license https://creativecommons.org/licenses/by-nc-nd/4.0/

The WestminsterResearch online digital archive at the University of Westminster aims to make the research output of the University available to a wider audience. Copyright and Moral Rights remain with the authors and/or copyright owners. 


\section{Theoretical Computer Science}

Branching-Time Logic ECTL\# and its tree-style one-pass tableau: Extending Fairness

Expressibility of ECTL+

$$
\text { --Manuscript Draft-- }
$$

\begin{tabular}{|l|l|}
\hline Manuscript Number: & TCS-D-19-00158R2 \\
\hline Article Type: & VSI:TIME 2018 \\
\hline Section/Category: & B - Logic, semantics and theory of programming \\
\hline Keywords: & Temporal logic, fairness, tableau, branching-time, one-pass tableau \\
\hline Corresponding Author: & Montserrat Hermo \\
\hline First Author: & Donostia-SanSebastián, Gipuzkoa SPAIN \\
\hline Order of Authors: & Alexander Bolotov \\
\hline & Alexander Bolotov \\
\hline & Montserrat Hermo \\
\hline & Paqui Lucio \\
\hline
\end{tabular}




\section{Response to Reviewers}

We would like to thank the reviewer 2 for his/her very valuable comments in this second feedback on our paper. Please find below an account of changes we have introduced following your comments and our own revision of the paper. We would also like to stress that during this latest revision the numbering of the definitions, propositions, etc has changed comparing with the previous version, so in our reply below, to avoid ambiguity, we will make clear which text from the previous version we have changed and note the relevant numbering. Kind regards Alexander Bolotov, Montserrat Hermo, Paqui Lucio.

\section{Responses to Referee \#2.}

Comment 1 - on the examples illustrating ECTL\# applicability. Regarding the expressivity relative to CTL and CTL*, the examples help but are far from convincing. It is clear that ECTL\# is more expressive than CTL, but the example doesn't do enough to demonstrate it's significance. The policy, a user may never repeat a password is easily expressed in CTL: $A G(\neg($ password $=p w) \rightarrow A G(\neg($ password $=p w)))$. I believe that this faithfully represents the property (assume that password $=p w$ sometime in the past) and is semantically equivalent to the formula given (I haven't proved this, but no counter example is immediately apparent). I agree that if passwords were replaced by some non-atomic property (perhaps some acknowledge-response protocol that can't be repeated) you would have a compelling example for application of ECTL\#. However, the password example is not convincing.

The zombie process doesn't help the motivation either. You refer to "the removal of a process from a table" as $R_{i d}$, but your formula is $\left(Z_{i d} U G R_{i d}\right)$. The description sounds like $R_{i d}$ should be an atomic event, but $R_{i d}$ seems to be synonymous with $A G R_{i d}$, which can be enforced with CTL.

Our reply to Comment 1 . In this latest version we have reconsidered an example representing the property which is directly expressible in our logic but is not directly expressible in weaker ones, such as CTL - see page 2 of the revised version, just above Figure 1. We envisage, however, that there may be a way to express this, and similar properties, in weaker logics, but 'indirectly', and with the price of the exponential blow up - due to the lack of the required syntactical constraints, that are given in our logic - ECTL\#.

In the revised version we updated the scenario with the 'password change' and got rid of another example - zombie processes, as we believe another example is not needed.

In details. 1.) let us note, that we now give a direct reference to the class of system properties that our logic tackles - 'a system process (property) $p$ holds until a system invariant $q$ becomes 
true'. This is reflected in the following sentence copied from page 2: 'The fairness constraint $\mathrm{A}(p \mathcal{U} \square q)$ reads as 'invariant $q$ is true along all paths of the computation except possibly their finite initial interval, where $p$ is true'. 2.) We consider a typical scenario of system properties related to password change giving an updated (comparing to the previous version) example (below is the citation from the revised text):

"For example, the following property specifies that whenever the user of an account is requested to change the current password, either it is changed to a fresh one, or the account is deactivated:

$\mathrm{A}\left(\left(P_{n}^{w} \mathcal{U} \square\left(R_{n} \Rightarrow \mathrm{A} \square\left(P_{n}^{w^{\prime}} \Rightarrow w \neq w^{\prime}\right)\right) \vee\left(\left(L_{n} \wedge P_{n}^{w}\right) \mathcal{U} \square\left(\left(R_{n} \wedge\left(\neg P_{n}^{w^{\prime}} \vee w^{\prime}=w\right)\right) \Rightarrow \neg L_{n}\right)\right)\right)\right.$

where $P_{n}^{w}\left(P_{n}^{w^{\prime}}\right)$ stands for the account $n$ has associated password $w\left(w^{\prime}\right) ; L_{n}$ stands for the account $n$ is live, and $R_{n}$ means the account number $n$ is requested to change the password,

Comment 2 - on the introduction of ECTL\# as a sublanguage of CTL*. Perhaps I was being a little too subtle suggesting it was economical. I do not consider the definitions given in this section to be well founded. The box operator is an abbreviation for an expression containing box. Typically, this would lead to some infinite expression. Unless we recognize that we have a special "abbreviation" box, that is different to the box that appears in the syntax of ECTL\#. But then, the semantics are given with respect to box. But it is not clear if the semantics should be interpreted as being for:

(a) the box that appears in the base syntax of ECTL\# as a subpart of an operator,

(b) the abbreviation $\square(\sigma \wedge \square \mathbf{F})$

(c) the recursive application $\square((\sigma \wedge \square(\mathbf{F} \wedge \square \mathbf{F})) \wedge \square \mathbf{F})$

I know it is intended to be (a), and I know they are all semantically equivalent. However, you cannot simply switch an operator from being an abbreviation to being a fundamental part of the langauge. It is lazy and prone to error. I strongly recommend presenting ECTL\# as a subsyntax of CTL*, because that is exactly what it is. The argument that the "significance" of ECTL\# justifies a presentation independent of CTL* is nonsensical. Alternatively, you could perhaps use $G$, and a different font for $U$ to distinguish these operators from the subsyntax used to present the semantics.

Our Reply to comment 2. In this revised version we did follow the referee's suggestion and introduced ECTL\# as a sublanguage of CTL*, thus, addressing referee's comments on $\square$ operator.

In detail: in Section 2 we first introduce CTL* syntax (Def. 1) and semantics (Def 2). Then we show how ECTL\# can be obtained as a sublogic of CTL* in Def 7 . Then, in Def 8 , we introduce the syntax of ECTL\# in terms of nnf - this will simplify the subsequent presentation of the tableaux technique. 
Comment 3 - on consistent set of formulae. I agree the concept of a consistent set of formulas is crucial for the paper. I simply object to the name you have chosen. In the definition you refer to a "syntactically consistent set of formulas". I suggest that you change the title of Definition 2 to be "Syntactically Consistent Set of Formulae" and then you just have to add one word to the definition of node at the top of page 11 (which is the only place the definition is used).

Our Reply to comment 3. We have introduced the changes suggested by the referee which reflected in Def 4, which is now also entitled 'Syntactically Consistent Set of Formulae'.

\section{Comment 4 - on cyclic Kripke structures.}

Definition 8 is still not right. There are formulas that are not satisfied by cyclic models, such as: $A G((E G p \rightarrow X E G \neg p) \wedge(E G \neg p \rightarrow X E G p))$ This is satisfied by a completely connected model consisting of two states 1,2 where $p$ is true at 1 and not true at 2. This is not a cyclic model because it contains the non-cyclic fullpath

$$
1,2,1,1,2,2,1,1,1,2,2,2,1,1,1,1,2,2,2,2, \ldots
$$

Furthermore, such a path must exist in every model that satisfies that formula. Therefore, it is not correct to say on line 315 "we can consider that any path in a model is cyclic...", and this has nothing to do with the finite model property (as can be seen from the above example). It doesn't affect the correctness of your structure because you can show that: "any formula satisfied in any model, will be satisfied in the bundled semantics on the model consisting of only the cyclic fullpaths of the model." This needs to be made clear. Finally, there is some strange grammar in the statement "...K is cyclic if its every fullpath is a path over.." should be "... K is cyclic if every fullpath of $K$ is a path over..."

Our reply to comment 4. As the reviewer correctly mentions, formula $A G((E G p \rightarrow$ $X E G \neg p) \wedge(E G \neg p \rightarrow X E G p))$ is satisfied by a completely connected model consisting of two states 1,2 where $p$ is true at 1 and not true at 2 . However, we can show that the structure with the only cyclic path

$$
1,2,1,2,1,2, \ldots
$$

is a model of the reviewer's formula, because the subformulae $E G p$ and $E G \neg p$ are always false in this model, hence the implications are trivially true everywhere.

In the revised version, we propose a better explanation of our point - see the text just below Def 6:

The fact that $C T L^{\star}$ satisfiability can be reduced to the emptiness problem for automata on infinite trees (see [22,23]), ensures that the (non-empty) collection of models of a given satisfiable CTL* formula can be obtained by infinitely unwinding (in any possible way) a finite graph. Hence, for any $\mathrm{CTL}^{\star}$ formula $\phi$, such 
that $\operatorname{Mod}(\phi) \neq \emptyset$, there always exists a model $\mathcal{K} \in \operatorname{Mod}(\phi)$ such that $\mathcal{K}$ is cyclic. Therefore, when speaking about the satisfiability in $\mathrm{CTL}^{\star}$ (hence ECTL\#) we can consider cyclic Kripke structures.

Note also, that to support our position, additionally to [22], we now include a new reference - [23].

\section{Comment 5 - on the terminology related to the closure under nnf}

I disagree with the terminology here. You are not showing ECTL\# is closed under nnf, you are showing that it is closed under negation. Incidently you have defined the semantics without negation, so the proposition is not well-founded. Again, this would be much easier if you defined the full semantics for CTL*, and showed that the subsyntax was closed under semantic negation.

Our reply to comment 5 Thanks to the reviewer's comment, we have introduced the formulation of ECTL\# - in Def 8 - in terms of nnf. We also explicitly say, just before Proposition 9, that this Proposition ensures that the set $\mathcal{F}_{\text {Prop }}$ is closed under negation.

\section{Comment 6 - on the statement that we can consider every path as cyclic path}

This comment first relates to our updates in the previous version stating that our changes in that version were

better, but still not correct As mentioned in point 6, there are necessarily models with noncyclic paths. We can say that there are models such that every satisfiable path formula will be satisfied by a cyclic path. However, it is lazy and incorrect to simply say we can consider every path to be cyclic.

Our reply to comment 6 Again, thanks to reviewer's comment we have introduced relevant changes in the revised version.

In the second paragraph of page 12 of the revised version, we now write:

The idea behind priorities is that a tableau branch represents a path in a cyclic Kripke structure that is a possible model for the input formula. Therefore, it consists of a (possibly empty) initial sequence of states followed by a looping-sequence.

\section{Comment 7 - on the technical report}

Thanks for these clarifications and improvements. I notice that you have referenced a tech report to justify the complexity of the tableaux. I think where proofs are cut short due to space restrictions you should consider possibly providing a full proof in a technical report also.

\section{Our reply to comment 7}

Yes, we understand this, and the technical report contains full proofs. 


\title{
Branching-Time Logic ECTL\# and its tree-style one-pass tableau: Extending Fairness Expressibility of ECTL ${ }^{+}$
}

\author{
Alexander Bolotov \\ University of Westminster, W1W 6UW, London, UK. \\ Montserrat Hermo and Paqui Lucio \\ University of the Basque Country, P. Manuel de Lardizabal, 1, 20018-San Sebastián, Spain.
}

\begin{abstract}
Temporal logic has become essential for various areas in computer science, most notably for the specification and verification of hardware and software systems. For the specification purposes rich temporal languages are required that, in particular, can express fairness constraints. For linear-time logics which deal with fairness in the linear-time setting, one-pass and two-pass tableau methods have been developed. In the repository of the CTL-type branching-time setting, the well-known logics ECTL and $\mathrm{ECTL}^{+}$were developed to explicitly deal with fairness. However, due to the syntactical restrictions, these logics can only express restricted versions of fairness. The logic $C T L^{\star}$, often considered as 'the full branching-time logic' overcomes these restrictions on expressing fairness. However, $\mathrm{CTL}^{\star}$ is extremely challenging for the application of verification techniques, and the tableau technique, in particular. For example, there is no one-pass tableau construction for $\mathrm{CTL}^{\star}$, while one-pass tableau has an additional benefit enabling the formulation of dual sequent calculi that are often treated as more 'natural' being more friendly for human understanding. These two considerations lead to the following problem - are there logics that have richer expressiveness than $E C T L^{+}$, allowing the formulation of a new range of fairness constraints with 'until' operator, yet 'simpler' than CTL*, and for which a one-pass tableau can be developed? Here we give a positive answer to this question, introducing a sub-logic of $\mathrm{CTL}^{\star}$ called ECTL\# , its tree-style one-pass tableau, and an algorithm for obtaining a systematic tableau, for any given admissible branching-time formulae. We prove the termination, soundness and completeness of the method. As tree-shaped one-pass tableaux are well suited for the automation and are amenable for the implementation and for the formulation of sequent calculi. Our results also open a prospect of relevant developments of the automation and implementation of the tableau method for ECTL ${ }^{\#}$, and of a dual sequent calculi.
\end{abstract}

Keywords: Temporal logic, fairness, tableau, branching-time, one-pass tableau.

\section{Introduction}

Temporal logic has become essential for the specification and verification of hardware and software systems. For the specification of the reactive and distributed systems, or, most recently, autonomous systems, the modelling of the possibilities 'branching' into the future is essential. Among important properties of these systems, so called fairness 5 properties are important. In the standard formalisation of fairness, operators $\diamond$ (eventually) and $\square$ (always) have been used: $\mathrm{A} \diamond \square p-$ ' $p$ ' is true along all computation paths except possibly their finite initial interval, where ' $\mathrm{A}$ ' is 'for all paths' quantifier, and $\mathrm{E} \square \diamond p-$ ' $p$ ' is true along a computation path at infinitely many states, where 'E' stands

\footnotetext{
Email addresses: A. Bolotov@westminster.ac.uk (Alexander Bolotov), \{montserrat.hermo, paqui.lucio\}@ehu.eus (Montserrat Hermo and Paqui Lucio)

${ }^{1}$ The author would like to thank the University of Westminster for supporting the sabbatical in 2017.

${ }^{2}$ These authors have been partially supported by Spanish Projects TIN2013-46181-C2-2-R and TIN2017-86727-C2-2-R, and by the University of the Basque Country under Project LoRea GIU15/30.
} 
for 'there exists a path' quantifier. Branching-time logics (BTL) here give us an appropriate reasoning framework, where the most used class of formalisms are 'CTL' (Computation Tree Logic) type logics. CTL itself requires every 10 temporal operator to be preceded by a path quantifier, thus, cannot express fairness. ECTL (Extended CTL) [1] enables simple fairness constraints but not their Boolean combinations. $E C T L^{+}[2]$ further extends the expressiveness of ECTL allowing Boolean combinations of temporal operators and ECTL fairness constraints (but not permitting their nesting). The logic $C T L^{\star}$, often considered as 'the full branching-time logic' overcomes these restrictions on expressing fairness. However, $C T L^{\star}$ is extremely challenging for the application of any known technique of automated 15 reasoning. Note that, unlike fair CTL [3] which, in tackling fairness, changes the underlying trees to those with 'fair paths' only, ECTL and ECTL ${ }^{+}$do not impose these changes.

From another perspective, the literature on fairness constraints, even in the linear-time setting, lacks the analysis of their formulation with the $\mathcal{U}$ ('until') operator. To the best of our knowledge, there are only a few research papers that raise or discuss the problem. For example, [4], introduces the logic LCTL, providing an extension of liveness 20 constraints by the "until" operator. However, LCTL belongs to 'Fair CTL-type' logics [5]. 'Generalised liveness assumptions, which allow to express that the conclusion $f_{2} \mathcal{U} f_{3}$ of a liveness assumption $\square\left(f_{1} \Rightarrow\left(f_{2} \mathcal{U} f_{3}\right)\right)$ has to be satisfied' are addressed in [6]. The $\mathcal{U}$ operator in the formulation of the fairness can also be found in [7] which considers the sequential composition of processes, providing the following example - the composition of processes $P_{1}$ and $P_{2}$ 'behaves as $P 1$ until its termination and then behaves as $P 2$ '. Finally, [8] utilises restricted linear-time 25 fairness constraints with $\mathcal{U}$ in the linear-time setting. We are not aware of any other analysis of fairness constraints in branching-time setting using the $\mathcal{U}$ operator and without restricting the underlying logic to be interpreted over the 'fair' paths. We bridge this gap, presenting the logic ECTL\# (we use \# to indicate some restrictions on concatenations of the modalities and their Boolean combinations). It is weaker than $C T L^{\star}$ but extends $E C T L^{+}$by allowing the combinations $\square(A \mathcal{U} B)$ or $A \mathcal{U} \square B$, referred to as modalities $\square \mathcal{U}$ and $\mathcal{U} \square$. This enables the formulation of stronger 30 fairness constraints in the branching-time setting. The fairness constraint $\mathrm{A}(p \mathcal{U} \square q)$ reads as 'invariant $q$ is true along all paths of the computation except possibly their finite initial interval, where $p$ is true'. For example, the following property specifies that whenever the user of an account is requested to change the current password, either it is changed to a fresh one, or the account is deactivated:

$$
\mathrm{A}\left(\left(P_{n}^{w} \mathcal{U} \square\left(R_{n} \Rightarrow \mathrm{A} \square\left(P_{n}^{w^{\prime}} \Rightarrow w \neq w^{\prime}\right)\right) \vee\left(\left(L_{n} \wedge P_{n}^{w}\right) \mathcal{U} \square\left(\left(R_{n} \wedge\left(\neg P_{n}^{w^{\prime}} \vee w^{\prime}=w\right)\right) \Rightarrow \neg L_{n}\right)\right)\right)\right.
$$

where $P_{n}^{w}\left(P_{n}^{w^{\prime}}\right)$ stands for the account $n$ has an associated password $w\left(w^{\prime}\right) ; L_{n}$ stands for the account $n$ is live, 35 and $R_{n}$ means the account number $n$ is requested to change the password, Note that formula (1) represents one of the difficult cases of ECTL\# structures - an A-disjunctive formula, see $\$ 2$

\begin{tabular}{|l|c|c|c|c|}
\hline $\mathcal{B}(\mathcal{U}, \circ)(\mathrm{CTL})$ extensions & $\mathrm{E}(\square \vee q)$ & $\mathrm{E}(\square \vee q \wedge \square \diamond r)$ & $\mathrm{A}((p \mathcal{U} \square q) \vee(s \mathcal{U} \square \neg r))$ & $\mathrm{A} \diamond(\circ p \wedge \mathrm{E} \circ \neg p)$ \\
\hline $\mathcal{B}(\mathcal{U}, \circ, \square \diamond)\left(\mathrm{ECTL}^{\prime}\right)$ & $\sqrt{ }$ & $X$ & $X$ & $X$ \\
$\mathcal{B}^{+}(\mathcal{U}, \circ, \square \diamond)\left(\mathrm{ECTL}^{+}\right)$ & $\sqrt{ }$ & $\sqrt{ }$ & $X$ & $X$ \\
$\mathcal{B}^{+}(\mathcal{U}, \circ, \mathcal{U} \square)\left(\mathrm{ECTL}^{\#}\right)$ & $\sqrt{ }$ & $\sqrt{ }$ & $\sqrt{ }$ & $X$ \\
$\mathcal{B}^{\star}(\mathcal{U}, \circ)\left(\mathrm{CTL}^{\star}\right)$ & $\sqrt{ }$ & $\sqrt{ }$ & $\sqrt{ }$ \\
\hline
\end{tabular}

Figure 1: Classification of CTL-type logics and their expressiveness

Figure 1, which utilises another temporal operator - 0 - 'at the next moment of time', places our logic in the hierarchy of BTL representing their expressiveness: logics are classified by using ' $\mathcal{B}$ ' for 'Branching', followed by the set of only allowed modalities as parameters; $\mathcal{B}^{+}$indicates admissible Boolean combinations of the modalities 40 and $\mathcal{B}^{\star}$ reflects 'no restrictions' in either concatenations of the modalities or Boolean combinations between them 3 Thus, $\mathcal{B}(\mathcal{U}, \circ)$ denotes the logic CTL. In this hierarchy ECTL\# is $\mathcal{B}^{+}(\mathcal{U}, \circ, \mathcal{U} \square)$.

We present a tree-style one-pass tableau for ECTL\# continuing the analogous developments in linear-time case [11, 12] and for CTL [11]. An indicative feature of this approach is a context-based tableau technique. Contextbased tableaux have dual sequent calculi due to their handling of eventualities exclusively by using logical rules.

\footnotetext{
${ }^{3}$ This notation goes back to [9], here we use its nice tuning by Nicolas Markey in [10]. In the last column we use a short $C T L^{\star}$ formula $\mathrm{A} \diamond(\circ p \wedge \mathrm{E} \circ \neg p)$, not expressible by weaker logics. We found this formula indicative for $\mathrm{CTL}^{\star}$ as its validity is directly linked to the limit closure property [9].
} 
To the best of our knowledge, BTL more expressive than CTL have not enjoyed the context-based tableau though other kinds of tableaux exist for these logics. There is a single-pass tableau for CTL that carries out an 'on the fly' eventualities checking (non-logical mechanism) following the Schwendimann's approach [13]. For CTL*, which definitely is a super-logic of ECTL\#, different other kinds of tableau-style methods exist, remarkably [14, 15, 16, 17, 18]. Since $C T L^{\star}$ is much more expressive than ECTL\#, such methods often utilise additional mechanisms (non only inference rules) to control loops, which are, for example, automata-theoretic-based mechanism [17]. This brings extra complexity, which is justified to handle the $\mathrm{CTL}^{\star}$ expressivity. However, simpler proofs could be obtained for a weaker logic such as ECTL\# . There are also extensions of the tableau methods to super-logics of CTL*. For example, [19] introduces a two-pass tableau method for a logic that is a multiagent extension of CTL*. Tree-style one-pass tableaux (without additional procedures for checking meta-logical properties) have dual (cut-free) sequent

55 calculi, see [12], enabling the construction of human-understandable proofs. In addition, these tableaux are well suited for the automation and are amenable for the implementation $4^{4}$ Our tableau is effectively an AND-OR tree where nodes are labelled by sets of state (see the definitions in $\$ 2$ formulae. There are difficult cases of ECTL ${ }^{\#}$ formulae that appear due to the enriched syntax: disjunctions of formulae in the scope of the A quantifier and conjunctions of formulae in the scope of the $\mathrm{E}$ quantifier. To tackle these cases, in addition to $\alpha-\beta$ rules, that are standard to the ableaux, we define novel $\beta^{+}$-rules which use the context to force the eventualities to be fulfilled as soon as possible.

Outline of the paper. The rest of this paper, an extended version of [21], includes more examples, explanations, and detailed proofs of the results. It commences with $\$ 2$ where we describe ECTL\# as a sublogic of CTL*. The formulation of the tableau method is given in $\$ 3$, where we define and explain tableau rules. A systematic tableau construction and relevant examples are introduced in $\$ 4$. The soundness and completeness of our tableau method 65 are proved in $\$ 5$ and in $\$ 6$, respectively; for the latter, we prove the refutational completeness and termination of the presented method. Finally, in $\$ 7$ we draw the conclusions and prospects of future work that the presented results open.

\section{The logic ECTL\#}

As ECTL\# is a sublogic of CTL* we first recall $C T L^{\star}$ syntax and semantics.

Definition 1 (Syntax of $\left.C T L^{\star}\right)$. Given Prop is a fixed set of propositions, and $p \in$ Prop, we define sets of state ( $\sigma$ ) and path $(\pi) \mathrm{CTL}^{\star}$ formulae over Prop as follows: $\sigma::=\mathbf{T}|p| \sigma_{1} \wedge \sigma_{2}|\neg \sigma| \mathrm{E} \pi$ and $\pi::=\sigma\left|\pi_{1} \wedge \pi_{2}\right| \neg \pi|\circ \pi|$ $\pi \mathcal{U} \pi \mid \square \pi$.

In $\mathrm{CTL}^{\star}$, and all BTL logics, well formed formulae are state formulae.

Definition 2 (Labelled Kripke structure). A Kripke structure, $\mathcal{K}$, is a triple $(S, R, L)$ where $S \neq \emptyset$ is a set of states, $R \subseteq S \times S$ is a total binary relation, called the transition relation, and $L: S \rightarrow 2^{\text {Prop }}$ is a labelling function.

75 A fullpath $x$ through a Kripke structure $\mathcal{K}$ is an infinite sequence of states $s_{0}, s_{1}, \ldots$ such that $\left(s_{i}, s_{i+1}\right) \in R$, for every $i \geq 0$. Let 'fullpaths $(\mathcal{K})$ ' be the set of all fullpaths in $\mathcal{K}$. Given a fullpath $x=s_{0}, s_{1}, \ldots, s_{k}, \ldots(k \geq 0)$, we denote its state $s_{k}$ by $x(k)$, its finite prefix by the sequence $x^{\leq k}=s_{0}, s_{1}, \ldots, s_{k}$ and the suffix path $x^{\geq k}=s_{k}, s_{k+1}, \ldots$. When a fullpath $x$ is given, instead of $x(k)$ we will often write $k$, referring to $k$ as 'a state index of $x$ '. If $x$ is a fullpath and $y$ is a path such that $y(0)=x(k)$, for some $k>0$, then the juxtaposition $x^{\leq k} y$ is a fullpath. Our Kripke structures are labelled directed graphs that correspond to Emerson's R-generable structures, i.e. the transition relation $R$ is suffix, fusion and limit closed [9]. For any $\mathcal{K}$, any $x \in$ fullpaths $(\mathcal{K})$ and any natural number $i$, the notation $\mathcal{K}\lceil x(i)$ denotes a Kripke structure with the set of states of $\mathcal{K}$ restricted to those that are $R$-reachable from $x(i)$.

Definition 3. Given the structure $\mathcal{K}=(S, R, L)$, the relation $=$, which evaluates path formulae in a given path $x$ and state formulae at the state index $i$ of the given path $x$, is defined bellow:

$$
\begin{array}{lll}
\mathcal{K}, x, i \models \mathbf{T} & \text { and } & \mathcal{K}, x, i \models p \text { iff } p \in L(x(i)) . \\
\mathcal{K}, x, i \models \neg \sigma & \text { iff } & \mathcal{K}, x, i \models \sigma \text { does not hold. } \\
\mathcal{K}, x, i \models \sigma_{1} \wedge \sigma_{2} & \text { iff } & \mathcal{K}, x, i \models \sigma_{1} \text { and } \mathcal{K}, x, i \models \sigma_{2} . \\
\mathcal{K}, x, i \models \mathrm{E} \pi & \text { iff } & \text { there exists a path } y \in \text { fullpaths }(\mathcal{K}\lceil x(i)) \text { such that } \mathcal{K}, y \models \pi .
\end{array}
$$

\footnotetext{
${ }^{4}$ An excellent survey of the seminal tableau techniques for temporal logics can be found in [20].
} 


$$
\begin{array}{lll}
\mathcal{K}, x \models \circ \pi & \text { iff } & \mathcal{K}, x^{\geq 1} \models \pi . \\
\mathcal{K}, x \models \neg \pi & \text { iff } & \mathcal{K}, x \models \pi \text { does not hold. } \\
\mathcal{K}, x \models \pi_{1} \wedge \pi_{2} & \text { iff } & \mathcal{K}, x \models \pi_{1} \text { and } \mathcal{K}, x \models \pi_{2} . \\
\mathcal{K}, x \models \pi_{1} \mathcal{U} \pi_{2} & \text { iff } & \text { there exists } k \geq i \text { such that } \mathcal{K}, x^{\geq k} \models \pi_{2} \text { and } \mathcal{K}, x^{\geq j} \models \pi_{1} \text { for all } j \in\{0, \ldots, k-1\} . \\
\mathcal{K}, x=\square \pi & \text { iff } & \mathcal{K}, x^{\geq j} \models \pi \text { for all } j \geq 0 .
\end{array}
$$

In addition, for any set $\Sigma$ of state formulae, $\mathcal{K}, x, i=\Sigma$ iff $\mathcal{K}, x, i \models \sigma$, for all $\sigma \in \Sigma$.

Many other usual operators can be derived from those introduced, in particular, the 'falsehood' constant $\mathbf{F} \equiv \neg \mathbf{T}$, and the disjunction operator $\varphi_{1} \vee \varphi_{2} \equiv \neg\left(\neg \varphi_{1} \wedge \neg \varphi_{2}\right)$, as well as the temporal operator $\diamond \pi \equiv \mathbf{T} \mathcal{U} \pi$ and the universal path quantifier $\mathrm{A} \pi \equiv \neg \mathrm{E} \neg \pi$. It is also known that $\square \pi \equiv \neg \diamond \neg \pi$ but, for technical convenience, we define it as a primitive operator. Let us recall some meta-logical concepts that are essential for the paper.

Definition 4 (Syntactically Consistent Set of Formulae). A set $\Sigma$ of state formulae $\sigma$ is syntactically consistent abbreviated as $\Sigma_{\top}$ if $\mathbf{F} \notin \Sigma$ and $\{\sigma, \neg \sigma\} \nsubseteq \Sigma$ for any $\sigma$; otherwise, $\Sigma$ is inconsistent denoted as $\Sigma_{\perp}$.

Definition 5 (Satisfiability). For a set of state formulae $\Sigma$, the set of its models, $\operatorname{Mod}(\Sigma)$, is formed by all triples $(\mathcal{K}, x, i)$ such that $\mathcal{K}, x, i=\Sigma$. $\Sigma$ is satisfiable $(\operatorname{Sat}(\Sigma))$ if $\operatorname{Mod}(\Sigma) \neq \emptyset$, otherwise $\Sigma$ is unsatisfiable $(\operatorname{UnSat}(\Sigma))$.

If $\operatorname{Mod}(\Sigma)=\operatorname{Mod}\left(\Sigma^{\prime}\right)$ then $\Sigma$ and $\Sigma^{\prime}$ are equivalent denoted as $\Sigma \equiv \Sigma^{\prime}$. For a set of state formulae $\Sigma$, if for any fullpath $x \in$ fullpaths $(\mathcal{K})$, we have $\mathcal{K}, x, 0 \models \Sigma$, then we simply write $\mathcal{K} \models \Sigma$.

Definition 6 (Cyclic Sequence, Cyclic Path and Cyclic Kripke structure). Let $z$ be a finite sequence of states $z=$ $s_{0}, s_{1}, \ldots, s_{j}$ such that, for every $0 \leq k<j,\left(s_{k}, s_{k+1}\right) \in R$. Then, $z$ is cyclic iff there exists $s_{i}, 0 \leq i \leq j$ 100 such that $\left(s_{j}, s_{i}\right) \in R$. Let $z$ be a finite cyclic sequence, the subsequence $s_{i}, \ldots, s_{j}$ of $z$ is called a loop and $s_{i}$ is called the cycling element. We denote the loop as $\left\langle s_{i}, \ldots, s_{j}\right\rangle^{\omega}$. A cyclic path over $z$ is an infinite sequence $\operatorname{path}(z)=s_{0}, s_{1}, \ldots, s_{i-1}\left\langle s_{i}, s_{i+1}, \ldots, s_{j}\right\rangle^{\omega}{ }^{5}$ A Kripke structure $\mathcal{K}$ is cyclic if every fullpath is a cyclic path over a cyclic sequence of states.

The fact that $C T L^{\star}$ satisfiability can be reduced to the emptiness problem for automata on infinite trees (see [22, 23]), ensures that the (non-empty) collection of models of a given satisfiable CTL* formula can be obtained by infinitely unwinding (in any possible way) a finite graph. Hence, for any $\mathrm{CTL}^{\star}$ formula $\phi$, such that $\operatorname{Mod}(\phi) \neq \emptyset$, there always exists a model $\mathcal{K} \in \operatorname{Mod}(\phi)$ such that $\mathcal{K}$ is cyclic. Therefore, when speaking about the satisfiability in $\mathrm{CTL}^{\star}$ (hence ECTL\#) we can consider cyclic Kripke structures.

Proposing a new logic, ECTL\#, we aim at defining a sublogic of $\mathrm{CTL}^{\star}$ that extends the ECTL ${ }^{+}$formulae $\square \diamond \sigma$ and $\diamond \square \sigma$ (where $\sigma$ means state formula), respectively, to $\square(\sigma \mathcal{U} \sigma)$ and $\sigma \mathcal{U} \square \sigma$.

Definition 7 (Syntax of ECTL\# ). The set of ECTL\# formulae, over a fixed set of propositions Prop, are formed according to the following restriction of the $\mathrm{CTL}^{\star}$ grammar in Definition 1 for path formulae (state formulae are the same): $\quad \pi::=\sigma\left|\pi_{1} \wedge \pi_{2}\right| \neg \pi|\circ \sigma| \sigma \mathcal{U} \sigma|\square \sigma| \sigma \mathcal{U}(\square \sigma) \mid \square(\sigma \mathcal{U} \sigma)$.

Note that the nesting of pure path formulae, totally unrestricted in $\mathrm{CTL}^{\star}$, is now restricted by the grammar cases: $\circ \sigma|\sigma \mathcal{U} \sigma| \square \sigma|\sigma \mathcal{U} \square \sigma| \square(\sigma \mathcal{U} \sigma)$. In particular, $a \mathcal{U} \square(b \wedge \square c)$ (where $a, b, c \in$ Prop) is not an ECTL\# formula because $b \wedge \square c$ is directly in the scope of the $\square$ but is not a state formula. For technical convenience, we assume that the tableau construction applies to the formulae in negation normal form (shortly, nnf). Therefore, we introduce here a grammar for the set of ECTL\# formulae that is closed under negation and requires the negation to apply to atomic propositions (instead of state and path formulae).

120 Definition 8 (Syntax of ECTL\# in nnf). Let Prop be a fixed set of propositions, let $\rho \in$ Prop and let Lit ::= $\mathbf{F}|\mathbf{T}|$ $\rho \mid \neg \rho$, be the set of literals. The set $\mathcal{F}_{\text {Prop }}$ of $\mathrm{ECTL}^{\#}$ formulae in nnf (over Prop) is given by the grammar:

$\sigma::=L i t\left|\sigma_{1} \wedge \sigma_{2}\right| \sigma_{1} \vee \sigma_{2}|\mathrm{E} \pi| \mathrm{A} \pi$

$\pi::=\pi_{1} \wedge \pi_{2}\left|\pi_{1} \vee \pi_{2}\right| \circ \sigma|\sigma \mathcal{U}(\square \sigma)| \square(\sigma \mathcal{U} \sigma)|\square(\sigma \vee \square \sigma)| \sigma \mathcal{U}(\sigma \wedge \diamond \sigma)$ where $\sigma$ means a state formula, $\pi$ means a path formula, and $\diamond \sigma$ abbreviates $\mathbf{\mathbf { U }} \mathcal{U} \sigma$.

${ }^{5}$ Cyclic paths are also known as ultimately periodic paths. 
The modified grammar is obtained by extending the state formulae grammar by $A \pi$-formulae and the path formulae grammar by $\square(\sigma \vee \square \sigma)$ and $\sigma \mathcal{U}(\sigma \wedge \diamond \sigma)$. Cases $\sigma \mathcal{U} \sigma$ and $\square \sigma$ are omitted because they respectively abbreviate $\sigma \mathcal{U}(\sigma \wedge \diamond \mathbf{T})$ and $\square(\sigma \vee \square \mathbf{F})$. Note that, for $a, b, c \in$ Prop, the formula $\square(a \vee \square(b \vee \square c))$ is not in $\mathcal{F}_{\text {Prop }}$ because $b \vee \square c$ is not a state formula. The following proposition ensures that the set $\mathcal{F}_{\text {Prop }}$ is closed under negation.

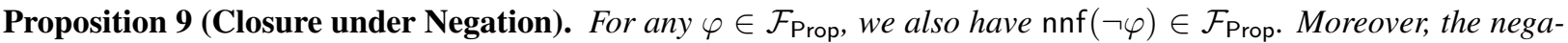
tion of a state (resp. path) formula is a state (resp. path) formula.

ProOF. By structural induction on the formulae, using the following equivalences (and well known classical ones):

$$
\begin{aligned}
& \text { 1. } \neg \mathrm{A} \varphi \equiv \mathrm{E} \neg \varphi \quad \text { 5. } \neg \square\left(\varphi_{1} \mathcal{U} \varphi_{2}\right) \equiv \diamond \square \neg \varphi_{2} \vee \diamond\left(\left(\neg \varphi_{1}\right) \wedge\left(\neg \varphi_{2}\right)\right) \\
& \text { 2. } \neg \mathrm{E} \varphi \equiv \mathrm{A} \neg \varphi \quad \text { 6. } \neg\left(\varphi_{1} \mathcal{U} \square \varphi_{2}\right) \equiv\left(\square \diamond \neg \varphi_{2}\right) \vee \diamond\left(\neg \varphi_{1} \wedge \diamond \neg \varphi_{2}\right) \\
& \text { 3. } \neg 0 \varphi \equiv \circ \neg \varphi \quad \text { 7. } \neg\left(\varphi_{1} \mathcal{U}\left(\varphi_{2} \wedge \diamond \varphi_{3}\right)\right) \equiv \square\left(\neg \varphi_{2} \vee \square \neg \varphi_{3}\right) \vee\left(\left(\neg \varphi_{2}\right) \mathcal{U}\left(\neg \varphi_{1} \wedge \neg \varphi_{2}\right)\right) \\
& \text { 4. } \neg \square \varphi \equiv \diamond \neg \varphi
\end{aligned}
$$

Equivalences 1-5 are very well known (e.g. [9]); the validity of 6 and 7 is easily established. It is also easy to see that 7 , when $\varphi_{3}$ is $\mathbf{T}$, is reduced to the known equivalence $\neg\left(\varphi_{1} \mathcal{U} \varphi_{2}\right) \equiv\left(\square \neg \varphi_{2}\right) \vee\left(\neg \varphi_{2} \mathcal{U}\left(\neg \varphi_{1} \wedge \neg \varphi_{2}\right)\right)$

For simplicity, we will write $\neg \varphi$ instead of $\operatorname{nnf}(\neg \varphi)$. Thus, $\neg \mathrm{A}(p \mathcal{U} \square q)$ represents $(\mathrm{E} \square \diamond \neg q) \vee \mathrm{E} \diamond(\neg p \wedge \diamond \neg q))$. 135 Also, for a finite set $\Delta=\left\{\varphi_{1}, \ldots, \varphi_{n}\right\}$, we let $\operatorname{nnf}\left(\neg \bigwedge_{i=1}^{n} \varphi_{i}\right)=\neg \Delta$.

\begin{tabular}{|l|l|l|}
\hline Type of a difficult case & A-disjunctive formula & E-conjunctive formula \\
\hline Example & $A(\circ q \vee \square r)$ & $E(\circ r \wedge q \mathcal{U} \square \neg p)$ \\
\hline Our representation & $A(\circ q, \square r)$ & $E(\circ r, q \mathcal{U} \square \neg p)$ \\
\hline
\end{tabular}

Figure 2: Difficult cases of temporal operators in the scope of path quantifiers

For ECTL\#, we identify the following difficult cases of the nesting and Boolean combinations of temporal operators in the scope of path quantifiers: A-disjunctive formula - disjunctions of temporal operators in the scope of $A$ and E-conjunctive formula - conjunctions of temporal operators in the scope of E. For convenience, we will, respectively, write $\mathrm{A}\left(\pi_{1}, \ldots, \pi_{n}\right)$ and $\mathrm{E}\left(\pi_{1}, \ldots, \pi_{n}\right)$, where $n \geq 1$, and if "," is in the scope of $\mathrm{A}$ it means $\vee$ while being in the scope of $E$ it means $\wedge$. Formulae serving as relevant examples in Figure 2 will be used to illustrate tableau, in Figure 6 . Note that any A-formula (E-formula) $\sigma$ can be transformed into an equivalent Boolean combination of A-disjunctive formulae $\mathrm{A}\left(\pi_{1}, \ldots, \pi_{n}\right)$ (E-conjunctive formulae $\mathrm{E}\left(\pi_{1}, \ldots, \pi_{n}\right)$ ), such that every $\pi_{i}(1 \leq i \leq n)$ is of one of the following: $\circ \sigma, \sigma \mathcal{U}(\sigma \wedge \diamond \sigma), \sigma \mathcal{U} \square \sigma, \square(\sigma \vee \square \sigma)$, and $\square(\sigma \mathcal{U} \sigma)$, and $\sigma$ stands for a state formula. For example, $\mathrm{A}(((\circ q) \wedge(\square \mathrm{E} \circ r)) \vee \circ p)$ is equivalent to $\mathrm{A}(\circ q) \wedge \mathrm{A}(\square \mathrm{E} \circ r, \circ p)$; and $\mathrm{E}(((\circ \mathrm{A} \circ r) \vee(q \mathcal{U} \square \mathrm{E} \neg p)) \wedge \circ q)$ is equivalent to $\mathrm{E}(\circ \mathrm{A} \circ r) \vee \mathrm{E}(q \mathcal{U} \square \mathrm{E} \neg p, \circ q)$. In what follows, $\mathrm{Q}$ abbreviates either of the path quantifiers. For a set of path formulae $\Pi=\left\{\pi_{1}, \ldots, \pi_{n}\right\}$, we write $\mathrm{Q} \Pi$ to denote $\mathrm{Q}\left(\pi_{1}, \ldots, \pi_{n}\right)$, and $\mathrm{Q} \circ \Pi$ to denote $\mathrm{Q}\left(\circ \pi_{1}, \ldots, \circ \pi_{n}\right)$. If $\Phi$ is an empty set of formulae it means $\mathbf{T}$ when $\Phi$ occurs in a conjunctive expression, and $\mathbf{F}$ in a disjunctive expression. In particular, when $\Pi$ is $\emptyset$ then $A \Pi$ is $\mathbf{F}$ and $E \Pi$ is $\mathbf{T}$. We write $\Sigma, \sigma$ to represent the set $\Sigma \cup\{\sigma\}$. We consider that every formula $\sigma \in \mathcal{F}_{\text {Prop }}$ is given in its equivalent 'negation normal form', $\operatorname{nnf}(\sigma)$.

\section{The Tableau Method}

\subsection{Preliminaries}

Definition 10 (Tableau, Consistent Node, Closed branch). A tableau for a set of ECTL\# state formulae $\Sigma$ is a labelled tree $T$, where nodes are $\tau$-labeled with sets of state formulae, such that the following two conditions hold:

$155 \quad$ (a) The root is labelled by the set $\Sigma$.

(b) Any other node $m$ is labelled with sets of state formulae as the result of the application of one of the rules in Figures 3, 4, 5 and 7to its parent node $n$. Given the applied rule is $R$, we term $m$ an $R$-successor of $n$.

A node $n$ of $T$ is consistent, abbreviated as $n_{\top}$, if $\tau(n)$ is a syntactically consistent set of formulae (see Def. 4), else $n$ is inconsistent, abbreviated as $n_{\perp}$. If a branch $b$ of $T$, contains $n_{\perp} \in b$, then $b$ is closed else $b$ is open. 


\begin{tabular}{|c|c|c|}
\hline & $\alpha$ & $S_{\alpha}$ \\
\hline$(\wedge)$ & $\sigma_{1} \wedge \sigma_{2}$ & $\left\{\sigma_{1}, \sigma_{2}\right\}$ \\
\hline$(\mathrm{E} \sigma)$ & $\mathrm{E}\left(\sigma_{1}, \ldots, \sigma_{n}, \Pi\right)$ & $\left\{\sigma_{1}, \ldots, \sigma_{n}, \mathrm{E} \Pi\right\}$ \\
\hline$(\mathrm{E} \square \mathcal{U})$ & $\mathrm{E}\left(\square\left(\sigma_{1} \mathcal{U} \sigma_{2}\right), \Pi\right)$ & $\left\{\mathrm{E}\left(\sigma_{1} \mathcal{U} \sigma_{2}\right.\right.$, o $\left.\left.\square\left(\sigma_{1} \mathcal{U} \sigma_{2}\right), \Pi\right)\right\}$ \\
\hline$(\mathrm{A} \square \mathcal{U})$ & $\mathrm{A}\left(\square\left(\sigma_{1} \mathcal{U} \sigma_{2}\right), \Pi\right)$ & $\left\{\mathrm{A}\left(\sigma_{1} \mathcal{U} \sigma_{2}, \Pi\right), \mathrm{A}\left(\circ \square\left(\sigma_{1} \mathcal{U} \sigma_{2}\right), \Pi\right)\right\}$ \\
\hline
\end{tabular}

Figure 3: ALPHA RULES. (Notation: $\sigma, \sigma_{i}$ stand for state formulae and $\Pi$ is a set of path formulae, possibly empty.)

\begin{tabular}{|c|c|c|c|}
\hline$\beta \_$Rule & $\beta$ & $k$ & $S_{\beta_{i}}(1 \leq i \leq k)$ \\
\hline$(\vee)$ & $\sigma_{1} \vee \sigma_{2}$ & 2 & $\begin{aligned} S_{\beta_{1}} & =\left\{\sigma_{1}\right\} \\
S_{\beta_{2}} & =\left\{\sigma_{2}\right\}\end{aligned}$ \\
\hline$(\mathrm{A} \sigma)$ & $\mathrm{A}\left(\sigma_{1}, \ldots, \sigma_{n}, \Pi\right)$ & $n+1$ & $\begin{array}{l}S_{\beta_{1}}=\left\{\sigma_{1}\right\} \\
\vdots \\
S_{\beta_{n}}=\left\{\sigma_{n}\right\} \\
S_{\beta_{n+1}}=\{\mathrm{A} \Pi\}\end{array}$ \\
\hline$(\mathrm{E} \square \sigma)$ & $\mathrm{E}\left(\square\left(\sigma_{1} \vee \square \sigma_{2}\right), \Pi\right)$ & 2 & $\begin{aligned} S_{\beta_{1}} & =\left\{\sigma_{1}, \mathrm{E}\left(\mathrm{\circ} \square\left(\sigma_{1} \vee \square \sigma_{2}\right), \Pi\right)\right\} \\
S_{\beta_{2}} & =\left\{\neg \sigma_{1}, \sigma_{2}, \mathrm{E}\left(\circ \square \sigma_{2}, \Pi\right)\right\}\end{aligned}$ \\
\hline$(\mathrm{E} \mathcal{U} \sigma)$ & $\mathrm{E}\left(\sigma_{1} \mathcal{U}\left(\sigma_{2} \wedge \diamond \sigma_{3}\right), \Pi\right)$ & 2 & $\begin{aligned} S_{\beta_{1}} & =\left\{\sigma_{2}, \mathrm{E}\left(\diamond \sigma_{3}, \Pi\right\}\right. \\
S_{\beta_{2}} & =\left\{\sigma_{1}, \mathrm{E}\left(\circ\left(\sigma_{1} \mathcal{U}\left(\sigma_{2} \wedge \diamond \sigma_{3}\right)\right), \Pi\right)\right\}\end{aligned}$ \\
\hline$(\mathrm{E} \mathcal{U} \square)$ & $\mathrm{E}\left(\sigma_{1} \mathcal{U} \square \sigma_{2}, \Pi\right)$ & 2 & $\begin{aligned} S_{\beta_{1}} & =\left\{\mathrm{E}\left(\square \sigma_{2}, \Pi\right)\right\} \\
S_{\beta_{2}} & =\left\{\sigma_{1}, \mathrm{E}\left(\circ\left(\sigma_{1} \mathcal{U} \square \sigma_{2}\right), \Pi\right)\right\}\end{aligned}$ \\
\hline$(\mathrm{E} \square \mathcal{U})$ & $\mathrm{E}\left(\square\left(\sigma_{1} \mathcal{U} \sigma_{2}\right), \Pi\right)$ & 2 & $\begin{array}{l}S_{\beta_{1}}=\left\{\sigma_{2}, \mathrm{E}\left(\mathrm{\circ} \square\left(\sigma_{1} \mathcal{U} \sigma_{2}\right), \Pi\right)\right\} \\
S_{\beta_{2}}=\left\{\sigma_{1}, \mathrm{E}\left(\mathrm{\circ}\left(\sigma_{1} \mathcal{U} \sigma_{2}\right), \Pi\right)\right\}\end{array}$ \\
\hline$(\mathrm{A} \square \sigma)$ & $\mathrm{A}\left(\square\left(\sigma_{1} \vee \square \sigma_{2}\right), \Pi\right)$ & 3 & $\begin{aligned} S_{\beta_{1}} & =\left\{\sigma_{1}, \mathrm{~A}\left(\mathrm{\circ} \square\left(\sigma_{1} \vee \square \sigma_{2}\right), \Pi\right)\right\} \\
S_{\beta_{2}} & =\left\{\neg \sigma_{1}, \sigma_{2}, \mathrm{~A}\left(\circ \square \sigma_{2}, \Pi\right)\right\} \\
S_{\beta_{3}} & =\{\mathrm{A} \Pi\}\end{aligned}$ \\
\hline$(\mathrm{A} \mathcal{U} \sigma)$ & $\mathrm{A}\left(\sigma_{1} \mathcal{U}\left(\sigma_{2} \wedge \diamond \sigma_{3}\right), \Pi\right)$ & 3 & $\begin{aligned} S_{\beta_{1}} & =\left\{\sigma_{2}, \mathrm{~A}\left(\diamond \sigma_{3}, \Pi\right)\right\} \\
S_{\beta_{2}} & =\left\{\sigma_{1}, \mathrm{~A}\left(\circ\left(\sigma_{1} \mathcal{U}\left(\sigma_{2} \wedge \diamond \sigma_{3}\right)\right), \Pi\right)\right\} \\
S_{\beta_{3}} & =\{\mathrm{A} \Pi\}\end{aligned}$ \\
\hline$(\mathrm{A} \mathcal{U} \square)$ & $\mathrm{A}\left(\sigma_{1} \mathcal{U} \square \sigma_{2}, \Pi\right)$ & 2 & $\begin{aligned} S_{\beta_{1}} & =\left\{\mathrm{A}\left(\square \sigma_{2}, \Pi\right)\right\} \\
S_{\beta_{2}} & =\left\{\sigma_{1}, \sigma_{2}, \mathrm{~A}\left(\circ\left(\sigma_{1} \mathcal{U} \square \sigma_{2}\right), \Pi\right)\right\}\end{aligned}$ \\
\hline
\end{tabular}

Figure 4: BETA RULES. (Notation: $\sigma, \sigma_{i}$ stand for state formulae, $\pi_{i}$ stand for path formulae, and $\Pi$ is a (possibly empty) set of path formulae.)

To make the presentation more transparent we give an informal overview of the tableau construction. Any tableau has a root-node that is exclusively labelled by a set of state formulae. To extend a node we apply one of $\alpha, \beta$ or $\beta^{+}$rule. The first two types of rules are standard to the tableau, and are essentially based on the fixpoint characterisation of $Q \square$ and $\mathrm{Q} \mathcal{U}$ modalities, while $\beta^{+}$rules are characteristic (and crucial!) for our construction. They tackle difficult cases of formulae in ECTL\#, and are related to our dedicated account of the eventualities. Namely, we treat an eventuality as occurring in some context, which, in turn, is a collection of all state formulae, called 'an outer context' or path formulae called 'an inner context'. As we will see, $\beta^{+}$rules use the context to force eventualities to be fulfilled as soon as possible. The $\alpha-\beta-\beta^{+}$rules apply repeatedly until they produce an inconsistent node $n_{\perp}$, or a node with the labels that already occurred within the path under consideration. In the former case the expansion of the given branch terminates with $n_{\perp}$ as its leaf. In the latter case, a repetitive node in the branch suggests that the input formula is satisfied forever, and we select another eventuality (if any) see $\$ 4.1$ Obviously, $n_{\perp}$ has an unsatisfiable $\tau(n)$ and is 
a 'deadlock' in the construction of a model. However, open branches do not necessarily give us a model. In particular, an open branch could be a prefix of a closed one. Later we introduce the notion of an expanded branch that enables the model construction. Once no more expansion rules are applicable to the given branch with the last node $n_{\top}$, we are ensured that $\tau(n)=\Sigma, \mathrm{A} \circ \Phi_{1}, \ldots, \mathrm{A} \circ \Phi_{n}, \mathrm{E} \circ \Psi_{1}, \ldots, \mathrm{E} \circ \Psi_{m}$, where $\Sigma$ is a set of literals. This labelling $\tau(n)$ is similar to a 'state' in the standard temporal tableau. Then the 'next-state' rule applies to generate the successors of $n$ with the labels that are arguments of all Qo modalities. The whole cycle of applying $\alpha-\beta-\beta^{+}$and 'next-state' rules is repeated until the tableau construction terminates. The nature of our rules ensures that the terminated tableau represents a model for the tableau input if all the leaves in a collection of branches, called a bunch, are consistent and all eventualities occurring in looping branches are fulfilled, otherwise, the tableau input is unsatisfiable.

$$
\text { (Q०) } \frac{\Sigma, \mathrm{A} \circ \Phi_{1}, \ldots, \mathrm{A} \circ \Phi_{\ell}, \mathrm{E} \circ \Psi_{1}, \ldots, \mathrm{E} \circ \Psi_{k},}{\mathrm{~A} \Phi_{1}, \ldots, \mathrm{A} \Phi_{\ell}, \mathrm{E} \Psi_{1} \& \ldots \& \mathrm{~A} \Phi_{1}, \ldots, \mathrm{A} \Phi_{\ell}, \mathrm{E} \Psi_{k}}
$$

Figure 5: NeXt-State Rule. (Notation: $\Sigma$ is a (possibly empty) set of literals, and $\Phi_{i}, \Psi_{i}$ are non-empty sets of formulae.)

\section{3.2. Alpha, Beta Rules and Next-State Rule}

The $\alpha$ - and $\beta$-rules are the most elementary rules of our tableau system. An $\alpha$-rule enlarges a branch with a node labelled by $\Sigma, \alpha$, by a successor node labelled by $\Sigma, S_{\alpha}$, where $S_{\alpha}$ is the set of formulae associated with $\alpha$ in Figure 3 . An $\alpha$-rule is represented as $\frac{\Sigma, \alpha}{\Sigma, S_{\alpha}}$ while $\beta$-rules as $\frac{\Sigma, \beta}{\Sigma, S_{\beta_{1}}|\cdots| \Sigma, S_{\beta_{k}}}$. $\beta$-rule splits a branch containing a node labelled by a set $\Sigma, \beta$ (where $\beta$ is one of the formulae of Figure 4), in $k$ new nodes each labelled by the corresponding $\Sigma, S_{\beta_{i}}$, according to Figure 4. The next-state rule (Q०), Figure 5 , also splits the branch into $k$ branches each of them rooted by node $n$ labelled by a set $\mathrm{A} \Phi_{1}, \ldots, \mathrm{A} \Phi_{\ell}, \mathrm{E} \Psi_{i}$, for $i \in\{1, \ldots, k\}$. This is the only rule of our system that splits branches in a conjunctive way. We use the symbol \& to represent the generation of AND-successors of node $n$. When $\ell=k=0$, the rule yields a unique new node labelled by the empty set. We assume that whenever $k=0$ and $\ell>0$, there exists a unique descendant labelled by $A \Phi_{1}, \ldots, A \Phi_{l}$.

190 Example 11. Let $n$ be a node such that $\tau(n)=\{a, \neg b, \mathrm{~A} \circ c, \mathrm{E} \circ p$, E० $\neg p, \mathrm{~A} \circ \square((\mathrm{E} \circ p) \wedge(\mathrm{E} \circ \neg p))\}$. Then the nextstate rule (Q०) applies to $n$ generating the following AND-successors of $n:\{\mathrm{A} c, p, \mathrm{~A} \square((\mathrm{E} \circ p) \wedge(\mathrm{E} \circ \neg p))\}$ and $\{\mathrm{A} c, \neg p, \mathrm{~A} \square((\mathrm{E} \circ p) \wedge(\mathrm{E} \circ \neg p))\}$. Note that $\mathrm{A} c$ requires the application of the $\beta$-rule $(\mathrm{A} \sigma)$ to be reduced to $c$.

\subsection{The Uniform Tableau}

In this subsection we explain how to construct a tableau where leaves are labelled by sets of formulae of a specific form - Uniform sets of state formulae.

Definition 12 (Elementary Set of ECTL\# State Formulae). A set of ECTL\# state formulae is elementary if and only if it is exclusively formed by literals and formulae of the form $Q \circ \Pi$.

Proposition 13. Any set of ECTL\# state formulae has a tableau $T$ such that all its leaves are labelled by elementary sets of state formulae.

PROOF. Repeatedly apply to every expandable node any applicable $\alpha$-rule or $\beta$-rule.

Example 14. Figure 6 depicts a tableau with elementary leaves for $\mathrm{A}(\circ q, \square r), \mathrm{E}(\circ r, q \mathcal{U} \square \neg p)$, Eoq. Recall that $\mathrm{A}(\circ q, \square r)$ is an abbreviation of $\mathrm{A}(\circ q, \square(r \vee \square \mathbf{F}))$.

Definition 15 (Basic Path/State Formula and Uniform Set of Formulae). Every ECTL\# path formula of the type $\circ \sigma, \sigma_{1} \mathcal{U}\left(\sigma_{2} \wedge \diamond \sigma_{3}\right), \sigma_{1} \mathcal{U}\left(\square \sigma_{2}\right), \square\left(\sigma_{1} \vee \square \sigma_{2}\right), \square\left(\sigma_{1} \mathcal{U} \sigma_{2}\right)$ is called basic. Every state formula $Q \Pi$ where $\Pi$ is a set of basic-path formulae is also called basic. A set of state formulae $\Sigma$ is uniform iff $\Sigma$ is exclusively formed by literals and basic state formulae, and $\Sigma$ contains at most one $\mathrm{E}$-conjunctive formula.

Proposition 16. Any set of ECTL\# state formulae $\Sigma$ has a tableau $T$ such that labels of all its leaves are uniform sets of state formulae. Moreover, each open branch of $T$ contains exactly one application of (Q०). 
ProOF. Use Proposition 13 to construct a tableau with all its leaves labelled by elementary sets of formulae. Then apply the rule (QO), to any relevant node and, finally, repeatedly apply (to every expandable node) the rules (E $\sigma$ ), $(\mathrm{A} \sigma),(\wedge)$, and $(\vee)$.

Definition 17 (Uniform Tableaux). For any set $\Sigma$ of $\mathrm{ECTL}^{\#}$ state formulae, the tableau for $\Sigma$ provided by Proposition 16 is denoted Uniform_Tableau $(\Sigma)$.

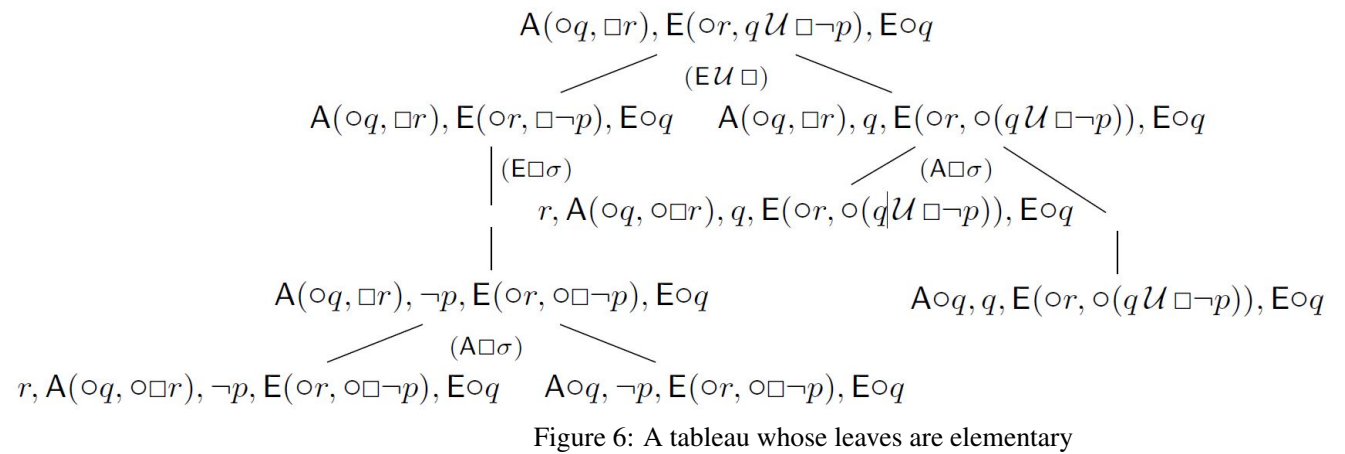

Example 18. Constructing a uniform tableau for the set $\{\mathrm{A}(\circ q, \square r), \mathrm{E}(\circ r, q \mathcal{U} \square \neg p), \mathrm{E} \circ q)\}$, we first obtain the 215 tableau in Figure 6. Then we apply the (Q०) rule enlarging each of the four branches and producing the following eight leaves, left to right (we refer to the node by its labels):
1. $\mathrm{A}(q, \square r), \mathrm{E}(r, \square \neg p)$
2. $\mathrm{A}(q, \square r), \mathrm{E} q \quad$ 3. $\mathrm{A} q, \mathrm{E}(r, \square \neg p)$
4. $\mathrm{A} q, \mathrm{E} q$
5. $\mathrm{A}(q, \square r), \mathrm{E}(r, q \mathcal{U} \square \neg p)$
6. $\mathrm{A}(q, \square r), \mathrm{E} q \quad$ 7. $\mathrm{A} q, \mathrm{E}(r, q \mathcal{U} \square \neg p)$
8. $\mathrm{A} q, \mathrm{E} q$

Then we apply the rules $(\mathrm{A} \sigma)$ and $(\mathrm{E} \sigma)$ : the first branch is split into $q, r, \mathrm{E} \square \neg p$ and $\mathrm{A} \square r, r, \mathrm{E} \square \neg p$; the second into $q$ and $\mathrm{A} \square r, q$; the third yields only a child $q, r, \mathrm{E} \square \neg p$; the fourth and the eighth yield only $q$; the fifth is split into two nodes $q, r, \mathrm{E}(q \mathcal{U} \square \neg p)$ and $\mathrm{A} \square r, r, \mathrm{E}(q \mathcal{U} \square \neg p)$; the sixth into $q$ and $\mathrm{A} \square r, q$; and the seventh yields the unique child $q, r, \mathrm{E}(q \mathcal{U} \square \neg p)$.

\subsection{The Beta-plus Rules}

In this subsection we extend our set of tableau rules with the new four rules named as $\beta^{+}$-rules (Figure 7 ). These rules, similarly to $\beta$-rules, also split a branch, but this time into a number of branches depending on the treated formula.

The rules for A-disjunctive formulae apply to a label $\Sigma, \beta$, where $\beta$ has the form $\mathrm{A}(\pi, \Pi)$, $\Pi$ is a set of basic-path formulae, and $\pi$ is either $\sigma \mathcal{U}(\sigma \wedge \diamond \sigma)$ or $\sigma \mathcal{U} \square \sigma$. The rule $(\mathrm{E} \mathcal{U} \sigma \square)^{+}$for E-conjunctive formulae applies to a set $\Sigma, \beta$, where $\beta$ has the form $\mathrm{E} \Pi$ and $\Pi$ is a set of basic-path formulae that contains at least one formula $\sigma \mathcal{U}(\sigma \wedge \diamond \sigma)$ or $\sigma \mathcal{U} \square \sigma$. The $\beta^{+}$-rules are the only rules in our system that make use of the so-called context for forcing the eventualities to be satisfied as soon as possible. The context is given by the sets $\Sigma$ containing state formulae and $\Pi$ containing path formulae. We name $\Sigma$ the outer context and $\Pi$ the inner context. The outer context is used by all the $\beta^{+}$-rules. The inner context is only needed to deal with formulae $A \Pi$. The following formula, $\varphi_{\Pi}$, introduced in Def. 19 is used to manage the inner context $\Pi$ in rules $(\mathrm{A} \mathcal{U} \sigma)^{+}$and $(\mathrm{A} \mathcal{U} \square)^{+}$.

Definition 19 (Formula $\varphi_{\Pi}$ for $\beta^{+}$-rules on A-disjunctive formulae). Let $\Pi$ be a set of basic path formulae. We define the formula $\varphi_{\Pi}$ to be the following disjunction of state formulae $\left(\varphi_{\Pi}\right.$ is $\mathbf{F}$, if the below disjunction is empty):

$$
\bigvee_{\square\left(\sigma_{1} \vee \square \sigma_{2}\right) \in \Pi}\left(\sigma_{1} \vee \sigma_{2}\right) \vee \bigvee_{\sigma_{1} \mathcal{U} \square \sigma_{2} \in \Pi} \sigma_{2} \vee \underset{\square\left(\sigma_{1} \mathcal{U} \sigma_{2}\right) \in \Pi}{\bigvee} \mathrm{E}\left(\diamond \sigma_{2}\right)
$$

It is worth noting that each $\beta^{+}$-rule, when applied to some formula of the form $\mathrm{Q}\left(\sigma_{1} \mathcal{U} \varphi, \Pi\right)$-where $\varphi$ could be $\sigma_{2} \wedge \diamond \sigma_{3}$ or $\square \sigma_{2}$ - generates one or more successors that contain a formula of the form $\mathrm{Q}\left(\circ\left(\left(\sigma_{1} \wedge \sigma\right) \mathcal{U} \varphi\right), \Pi\right)$ where $\sigma$ depends on both the inner and the outer context, and is defined depending on whether $\mathrm{Q}$ is $\mathrm{E}$ or $\mathrm{A}$. We call $\left(\sigma_{1} \wedge \sigma\right) \mathcal{U} \varphi$ the next-step variant of $\sigma \mathcal{U} \varphi$. Example 20 illustrates the main ideas behind the application of $\beta^{+}$-rules $(\mathrm{A} \mathcal{U} \sigma)^{+}$and $(\mathrm{A} \mathcal{U} \sigma)$. 
Figure 7: BetA-PLuS RULES. (Notation: $\sigma, \sigma_{i}$ stand for state formulae, $\Sigma$ is a (possibly empty) set of state formulae, $\Pi$ is a (possibly empty) set of basic-path formulae. Formula $\varphi_{\Pi}$ is defined in Def. 19 We denote by $\Pi_{\mathcal{U}}$ the set of all formulae in $\Pi$ that have the forms $\sigma_{1} \mathcal{U}\left(\sigma_{2} \wedge \diamond \sigma_{3}\right)$ and $\sigma_{1} \mathcal{U} \square \sigma_{2} . \Pi_{\mathcal{U}}$ is enumerated as $\left\{\pi_{1}, \ldots, \pi_{n}\right\}$ for $n \geq 1$, and $\Pi^{-i}=\Pi \backslash\left\{\pi_{i}\right\}$.)

Example 20. The $\beta^{+}$-rules $(\mathrm{A} \mathcal{U} \sigma)^{+}$applies to one selected formula with exactly one marked eventuality. Consequently, the $(\mathrm{A} \mathcal{U} \sigma)$-rule applies to all the eventualities (in the selected formula) except to the marked one.

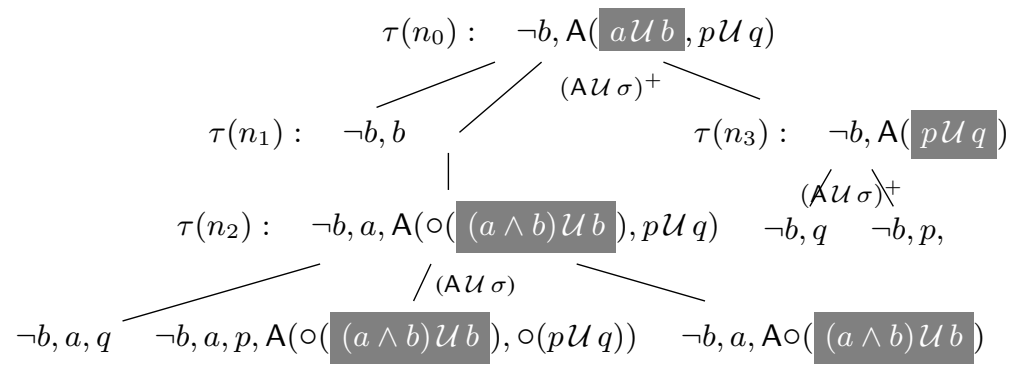

Figure 8: Application of $(\mathrm{A} \mathcal{U} \sigma)^{+}$and $(\mathrm{A} \mathcal{U} \sigma)$ rules to $\{\neg b, \mathrm{~A}(a \mathcal{U} b, p \mathcal{U} q)\}$

In Figure 8 the marked eventualities are in gray boxes. Assume aU $b$ is the marked eventuality. Then, the $(\mathrm{A} \mathcal{U} \sigma)^{+}$rule can be applied to $a \mathcal{U} b)$ with outer context $\Sigma=\{\neg b\}$ and inner context, $\Pi=\{p \mathcal{U} q\}$. According to Definition 19 . $\varphi_{\Pi}$ is $\mathbf{F}$. Therefore, $S_{\Sigma, \beta_{1}}^{+}=\{b\}, S_{\Sigma, \beta_{3}}^{+}=\{\mathrm{A}(p \mathcal{U} q)\}$, and $S_{\Sigma, \beta_{2}}^{+}=\{a, \mathrm{~A}(\circ((a \wedge b) \mathcal{U} b), p \mathcal{U} q)\}$ respectively generate nodes $n_{1}, n_{2}$ and $n_{3}$. In node $n_{2}$, the $(\mathrm{A} \mathcal{U} \sigma)$-rule is applied to $p \mathcal{U} q$. That produces three new nodes. Regarding node $n_{3}$, the marked eventuality disappears. Then, a new selection is made and $p \mathcal{U} q$ is marked. Consequently, $(\mathrm{A} \mathcal{U} \sigma)^{+}$-rule is applied with outer context $\Sigma=\{\neg b\}$. The inner context is the empty set. Note that all leaves in Figure 8 are elementary. Hence, the construction of the tableau, would continue applying the next-state rule (Q०). 


\subsection{Simplification Rules}

A large set of simplification rules can be used to reduce the tableau construction. Here we only mention those that are essential for termination. First, to stop the growth of the subformula $\sigma$ in the successive next-step variants $\left(\sigma_{1} \wedge \sigma\right) \mathcal{U} \varphi$, we use trivial simplification rules such as $\varphi \wedge \varphi \longrightarrow \varphi$ and $\varphi \vee \varphi \longrightarrow \varphi$, as well as classical subsumptions rules. Second, to simplify the detection of equal labels (for looping in tableau branches) we use the following (subsumption-based) rules:

$(\sqsubset \mathrm{E} \square \mathcal{U}) \mathrm{E}\left(\sigma_{1} \mathcal{U} \sigma_{2}, \square\left(\sigma_{1} \mathcal{U} \sigma_{2}\right), \Pi\right) \rightarrow \mathrm{E}\left(\square\left(\sigma_{1} \mathcal{U} \sigma_{2}\right), \Pi\right)$.

$255(\sqsubset \mathrm{A} \square \mathcal{U})$ If $\Pi^{\prime} \subseteq \Pi$ then $\mathrm{A}\left(\sigma_{1} \mathcal{U} \sigma_{2}, \Pi\right) \wedge \mathrm{A}\left(\square\left(\sigma_{1} \mathcal{U} \sigma_{2}\right), \Pi^{\prime}\right) \rightarrow \mathrm{A}\left(\square\left(\sigma_{1} \mathcal{U} \sigma_{2}\right), \Pi^{\prime}\right)$.

Finally, to prevent the duplications of the original eventuality $\sigma_{1} \mathcal{U} \sigma_{2}$ and its successive new-step variants by rules $(\mathrm{Q} \square \mathcal{U})$ and $(\mathrm{Q} \mathcal{U} \sigma)^{+}$, and to ensure termination, we use the following (subsumption-based) simplification rules:

$(\sqsubset \mathrm{A} \sigma \mathcal{U}) \sigma_{2} \wedge \mathrm{A}\left(\sigma_{1} \mathcal{U} \sigma_{2}, \Pi\right) \longrightarrow \sigma_{2}$.

$(\sqsubset \mathrm{E} \mathcal{U} \sigma) \mathrm{E}\left(\left(\sigma_{1} \wedge \sigma\right) \mathcal{U} \varphi, \sigma_{1} \mathcal{U} \varphi, \Pi\right) \longrightarrow \mathrm{E}\left(\left(\sigma_{1} \wedge \sigma\right) \mathcal{U} \varphi, \Pi\right)$

${ }_{260}(\sqsubset \mathrm{A} \mathcal{U} \sigma)$ If $\Pi^{\prime} \subseteq \Pi$ then $\mathrm{A}\left(\left(\sigma_{1} \wedge \sigma\right) \mathcal{U} \varphi, \Pi^{\prime}\right) \wedge \mathrm{A}\left(\sigma_{1} \mathcal{U} \varphi, \Pi\right) \rightarrow \mathrm{A}\left(\left(\sigma_{1} \wedge \sigma\right) \mathcal{U} \varphi, \Pi^{\prime}\right)$.

\subsection{The role of $\varphi_{\Pi}$ in the Beta-plus Rules}

Let us consider a set of formulae $\Phi=\Sigma, \mathrm{A}(\sigma \mathcal{U} \varphi, \Pi)$. A model, $\mathcal{K}$, of $\Phi$ could satisfy $\mathrm{A}(\sigma \mathcal{U} \chi, \Pi)$ because each fullpath of $\mathcal{K}$ satisfies either $\sigma \mathcal{U} \chi$ or some formula $\pi \in \Pi$. The next-step variant of $\sigma \mathcal{U} \chi$ is $\left.\circ\left(\sigma \wedge\left(\neg \Sigma \vee \varphi_{\Pi}\right)\right) \mathcal{U} \chi\right)$, which makes $\neg \Sigma$ or $\varphi_{\Pi}$ satisfiable before $\chi$ is satisfied. The former produces open branches where $\chi$ is fulfilled as oon as possible, whereas the latter produces open branches that satisfy some of the $\pi \in \Pi$. Therefore, $\varphi_{\Pi}$ allows to generate a model from a branch in which $\sigma \mathcal{U} \varphi$ is not fulfilled, and some $\pi \in \Pi$ is satisfied. Example 21 illustrates these ideas from the constructive view, i.e when we construct a tableau for a formula $\mathrm{A}\left(\pi_{1}, \ldots, \pi_{n}\right)$.

$$
\begin{aligned}
& \tau\left(n_{1}\right): \mathrm{A}(a \mathcal{U} b, \square c, r \mathcal{U} \square s, \square(p \mathcal{U} q)) \\
& \text { | }(\mathrm{A} \mathcal{U} \sigma)^{+} \\
& \tau\left(n_{2}\right): \quad a, \mathrm{~A}(\circ \overbrace{((a \wedge(c \vee s \vee \mathrm{E} \vee q)) \mathcal{U} b)}^{\alpha_{1}}, \square c, r \mathcal{U} \square s, \square(p \mathcal{U} q)) \\
& \text {, 一 } \quad(\mathrm{A} \square \sigma)+(\mathrm{A} \mathcal{U} \square)+\mathrm{A} \square \mathcal{U})+(\mathrm{A} \mathcal{U} \sigma)+(\mathrm{Q} \circ)+(\sqsubset \mathrm{A} \square \mathcal{U}) \\
& \tau\left(n_{3}\right): \mathrm{A}\left(\alpha_{1}, \square c,(r \mathcal{U} \square s), \square(p \mathcal{U} q)\right) \\
& \text { | }(\mathrm{A} \mathcal{U} \sigma)^{+} \\
& \tau\left(n_{4}\right): a,(a \wedge(c \vee s \vee \mathrm{E} \diamond q)), \mathrm{A}\left(\circ\left(\alpha_{1}\right), \square c, r \mathcal{U} \square s, \square(p \mathcal{U} q)\right) \\
& (\mathrm{A} \square \sigma)+(\mathrm{A} \mathcal{U} \square)+\mathrm{A} \square \mathcal{U})+(\mathrm{A} \mathcal{U} \sigma)+(\wedge)+(\vee) \\
& \tau\left(n_{5}\right): a, q, c, r, p, \mathrm{~A}\left(\mathrm{o}^{\prime}\left(\alpha_{1}\right), \circ \square c, \circ(r \mathcal{U} \square s), \circ(p \mathcal{U} q)\right), \mathrm{A}\left(\circ\left(\alpha_{1}\right), \circ \square c, \circ(r \mathcal{U} \square s), \circ \square(p \mathcal{U} q)\right) \\
& \mid(\mathrm{Q} \circ)+(\sqsubset \mathrm{A} \square \mathcal{U}) \\
& \tau\left(n_{6}\right): \mathrm{A}\left(\alpha_{1}, \square c,(r \mathcal{U} \square s), \square(p \mathcal{U} q)\right)
\end{aligned}
$$

Figure 9: A branch of a tableau for $\mathrm{A}(a \mathcal{U} b, \square c, r \mathcal{U} \square s, \square(p \mathcal{U} q)$.

Example 21. Let $\Pi=\{\square c, r \mathcal{U} \square s, \square(p \mathcal{U} q)\}$, and consider an application of the rule $(\mathrm{A} \mathcal{U} \sigma)^{+}$to the formula $\mathrm{A}(a \mathcal{U} b, \Pi)$, where $a, b, c, p, q, r, s \in$ Prop (see Figure 9). The outer context, namely $\Sigma$, is empty and the inner context is $\Pi$. Then $\neg \Sigma$ is $\mathrm{F}$ and $\varphi_{\Pi}=c \vee s \vee \mathrm{E} \vee q$. Hence, the second child, namely $S_{\Sigma, \beta_{2}}^{+}$, raised by the application of $(\mathrm{A} \mathcal{U} \sigma)^{+}$is labelled by $\left\{a, \mathrm{~A}\left(\circ \alpha_{1}, \Pi\right)\right\}$ where $\alpha_{1}=\left(a \wedge \varphi_{\Pi}\right) \mathcal{U} b=(a \wedge(c \vee s \vee \mathrm{E} \vee q) \mathcal{U} b$. Then, Uniform_Tableau applies the (corresponding) rules to $\square c, r \mathcal{U} \square s, \square(p \mathcal{U} q)$, and, finally, (Q०) and the simplification rule ( $\square \mathrm{A} \square \mathcal{U})$, obtaining the node $n_{3}$. Now, one of the leaves raised by Uniform_Tableau is the fifth node; by applying here (Q०) and $(\sqsubset \mathrm{A} \square \mathcal{U})$ we obtain the last node $n_{6}$ such that $\tau\left(n_{6}\right)=\tau\left(n_{3}\right)$. This branch represents a model of the initial 275 A-disjunctive formula where both disjuncts $\square(p \mathcal{U} q)$ and $\square c$ are satisfied, though the other two disjuncts are not. Indeed, it represents the model $\{a, c, r, p\},(\{a, c, r, p, q\})^{\omega}$.

\section{Systematic Tableau Construction}

In this section we define an algorithm, $\mathcal{A}^{\text {sys }}$, that constructs a systematic tableau and illustrate its performance with some examples. Recall that due to the rule $(\mathrm{Q} O)$, any open tableau should have a collection of open branches 
including all the (Q०)-successors of any node labelled by an elementary set of formulae. These collections of branches are called bunches. Any open bunch of the systematic tableau, constructed by the algorithm $\mathcal{A}^{\text {sys }}$ introduced in this section, enables the construction of a model for the initial set of formulae.

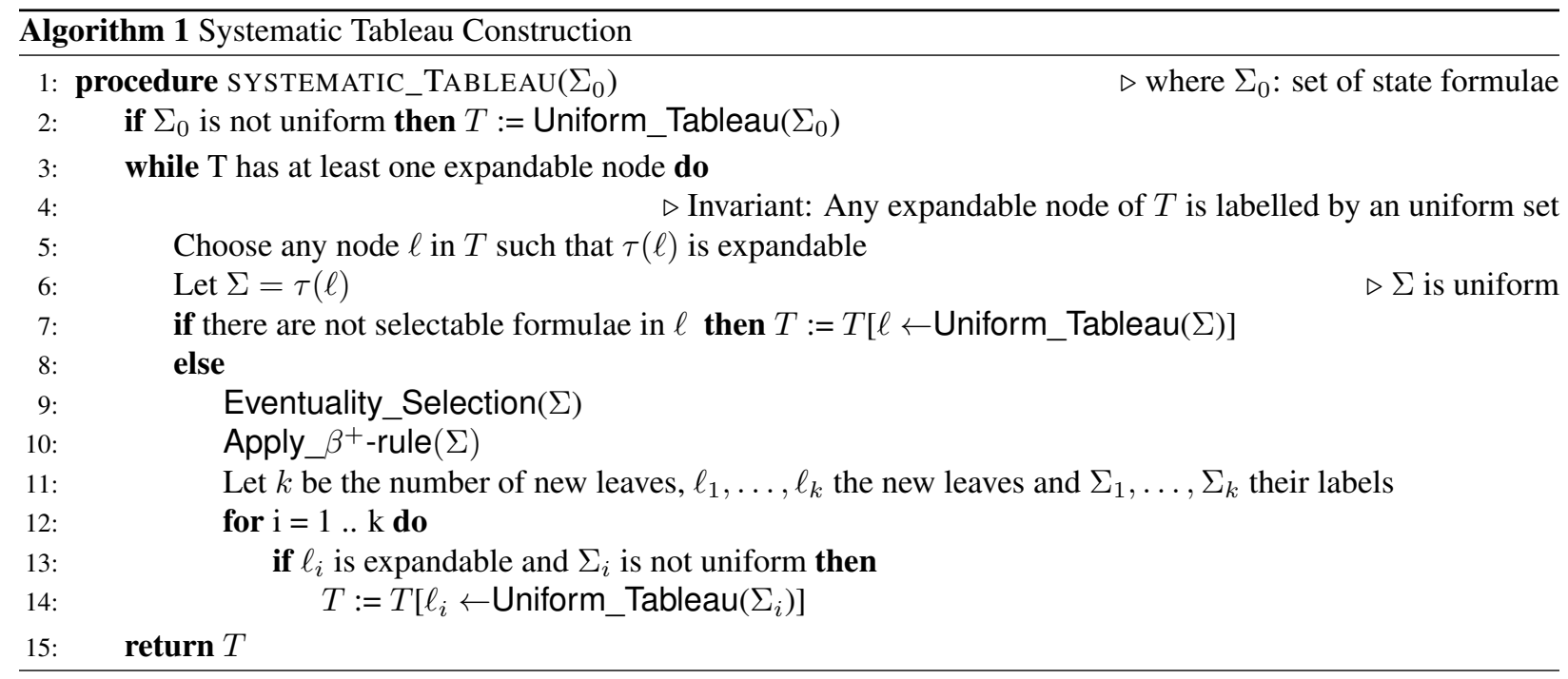

\subsection{The Algorithm}

The algorithm $\mathcal{A}^{\text {sys }}$ constructs an expanded tableau (see Definition 37) for the given input. $\mathcal{A}^{\text {sys }}$ applied to the input $\Sigma_{0}$, denoted as $\mathcal{A}^{\text {sys }}\left(\Sigma_{0}\right)$, returns a systematic tableau $\mathcal{A}_{\Sigma_{0}}^{\text {sys }}$. Intuitively, 'expanded' means 'complete' in the sense that any possible rule has been already applied at every node. Though the best way to implement this algorithm is a depth-first construction, for clarity, we formulate it as a breadth-first construction of a collection of subtrees. The procedure Uniform_Tableau, in the above Algorithm 1 1 was introduced in Definition 17 along with the notion of a uniform set of state formulae. The notation $T_{1}\left[\ell \leftarrow T_{2}\right]$ stands for the tableau $T_{1}$ where the expandable $\ell$ is substituted by the tableau $T_{2}$. In particular, $T[\ell \leftarrow$ Uniform_Tableau $(\Sigma)]$ is the tableau $T$ where the expandable $\ell$ is substituted by the Uniform_Tableau $(\Sigma)$. Next, we define the other two auxiliary procedures: Eventuality_Selection and Apply_ $\beta^{+}$-rule, as well as the related concepts of selectable formula, non-expandable node and eventualitycovered branch. From now on any basic path formula that is either $\sigma_{1} \mathcal{U}\left(\sigma_{2} \wedge \diamond \sigma_{3}\right)$ or $\sigma_{1} \mathcal{U} \square \sigma_{2}$ or $\square\left(\sigma_{1} \mathcal{U} \sigma_{2}\right)$ is called an eventuality. It is worth noting that $\bigcirc \pi$ is not called an eventuality in this setting.

Definition 22 (Selectable Formula). A formula is selectable if it is a QП and $\Pi$ contains at least one eventuality.

Procedure Eventuality_Selection chooses formula $Q \Pi$ and if $Q=A$ then the procedure also marks one eventuality, according to the priorities of selection and marking in Definition 24 We denote by $\pi_{\mathcal{U}}$ the marked eventuality in the selected formula AП.

Procedure Apply_ $\beta^{+}$-rule $(\Sigma)$ applies the corresponding rules depending on the selected formula Q $\Pi$ and on the 300 marked eventuality in the case $\mathrm{Q}=\mathrm{A}$ :

- If $\mathrm{Q}=\mathrm{A}$ and $\sigma_{1} \mathcal{U}\left(\sigma_{2} \wedge \diamond \sigma_{3}\right) \in \Pi$ is the marked eventuality, then apply $(\mathrm{A} \mathcal{U} \sigma)^{+}$

- If $\mathrm{Q}=\mathrm{A}$ and $\sigma_{1} \mathcal{U} \square \sigma_{2} \in \Pi$ is the marked eventuality, then apply $(\mathrm{A} \mathcal{U} \square)^{+}$

- If $\mathrm{Q}=\mathrm{A}$ and $\square\left(\sigma_{1} \mathcal{U} \sigma_{2}\right) \in \Pi$ is the marked eventuality, then first apply the rule $(\mathrm{A} \square \mathcal{U})$ and then the rule $(\mathrm{A} \mathcal{U} \sigma)^{+}$with the marked eventuality $\sigma_{1} \mathcal{U} \sigma_{2}$.

- If $\mathrm{Q}=\mathrm{E}$ and $\Pi$ contains at least one $\sigma_{1} \mathcal{U}\left(\sigma_{2} \wedge \diamond \sigma_{3}\right)$ or one $\sigma_{1} \mathcal{U} \square \sigma_{2}$ then apply $(\mathrm{E} \mathcal{U} \sigma \square)^{+}$

- If $\mathbf{Q}=\mathrm{E}$ and $\Pi$ contains at least one $\square\left(\sigma_{1} \mathcal{U} \sigma_{2}\right)$ (but none $\sigma_{1} \mathcal{U}\left(\sigma_{2} \wedge \diamond \sigma_{3}\right)$ and none $\left.\sigma_{1} \mathcal{U} \square \sigma_{2}\right)$, then first apply the rule $(\mathrm{E} \square \mathcal{U})$ to every $\square\left(\sigma_{1} \mathcal{U} \sigma_{2}\right)$ and then the rule $(\mathrm{E} \mathcal{U} \sigma \square)^{+}$.

Each application of a $\beta^{+}$-rule on the selected $A \Pi$ introduces a next-step variant of the marked eventuality and each application of a $\beta^{+}$-rule on the selected EП introduces a next-step variant for each $\sigma_{1} \mathcal{U}\left(\sigma_{2} \wedge \diamond \sigma_{3}\right)$ and each $\sigma_{1} \mathcal{U} \square \sigma_{2}$.

The call Eventuality_Selection $(\Sigma)$ keeps the selection of the formula EП such that $\Pi$ contains at least one $\sigma \mathcal{U}(\sigma \wedge \diamond \sigma)$ or $\sigma \mathcal{U} \square \sigma$, or keeps the selection of the formula $A \Pi \in \Sigma$ which contains the next-step variant of 
the marked eventuality, or selects a new formula $A \Pi \in \Sigma$ that contains an eventuality. The latter can happen for three reasons. When formulae $\mathrm{E} \Pi$ do not contain any $\sigma \mathcal{U}(\sigma \wedge \diamond \sigma)$ nor $\sigma \mathcal{U} \square \sigma$, or there is no marked eventuality in formulae $\mathrm{A} \Pi$, or when there is one, the node $\ell,(\Sigma=\tau(\ell))$ is a loop-node (see Definition 25), and the branch 315 from the root-node to $\ell$ is not eventuality-covered (see Definition 26. In this case, a new selection should be made because there are eventualities that have never been marked but they should be. This way we introduce the term eventuality-covered. When a branch is eventuality-covered, its leaf is a loop-node and we are sure that, along the loop, at least some eventuality in each A-disjunctive formula and all eventualities in each E-conjunctive formula have been fulfilled. Consequently, the branch is an expanded open branch (see Definition 37) and represents a path in a possible

320 model. It is worth mentioning that the only requirement for a branch to be eventuality-covered is to mark all necessary eventualities. The fact that formulae EП are kept selected whereas they contain some eventuality and formulae AП are kept selected where the next-step variant of the marked eventuality is kept marked ensures that every eventuality in $\mathrm{E} \Pi$ and at least one in each $A \Pi$ is fulfilled.

When making the selection, priorities are used as stated in Definition 24. The idea behind priorities is that a 325 tableau branch represents a path in a cyclic Kripke structure that is a possible model for the input formula. Therefore, it consists of a (possibly empty) initial sequence of states followed by a looping-sequence. Selectable formulae are classified into two sets - those of the highest priority and those of the lowest priority. The non-looping initial sequence is the first part of the model, hence we firstly select formulae AП where $\Pi$ is exclusively formed by formulae of the

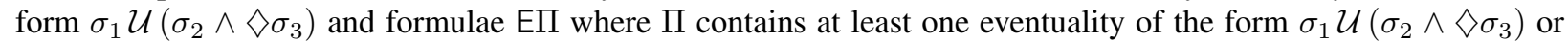
${ }_{330} \sigma_{1} \mathcal{U} \square \sigma_{2}$. These are the highest priority formulae, which cannot produce a loop. When one of the former formulae A $\Pi$ is selected, one of the $\sigma_{1} \mathcal{U}\left(\sigma_{2} \wedge \diamond \sigma_{3}\right)$ is marked, namely $\pi$. By means of the rule $(\mathrm{A} \mathcal{U} \sigma)^{+}$, in a finite number of steps, either the branch close or $\pi$ is either fulfilled (note that in the first branch $\mathrm{A}\left(\diamond \sigma_{3}, \Pi\right)$ is also of the highest priority) or deleted (the third branch of $\left.(\mathrm{A} \mathcal{U} \sigma)^{+}\right)$. In any case the original formulae $\mathrm{A} \Pi$ disappears. When one of the latter formulae $\mathrm{E} \Pi$ is selected, the successive applications of the rule $(\mathrm{E} \mathcal{U} \sigma \square)^{+}$ensure (excluding the case when the 335 branch closes) the fulfillment of all $\sigma_{1} \mathcal{U}\left(\sigma_{2} \wedge \diamond \sigma_{3}\right)$ or $\sigma_{1} \mathcal{U} \square \sigma_{2}$ in a finite number of states. Note that $\mathrm{E}\left(\diamond \sigma_{3}, \Pi\right)$ is also of the highest priority. Once such formulae are fulfilled, all formulae $\sigma_{1} \mathcal{U}\left(\sigma_{2} \wedge \diamond \sigma_{3}\right)$ have disappeared from the E-conjunctive formula, whereas $\square \sigma_{2}$ remains in the conjunction for all $\sigma_{1} \mathcal{U} \square \sigma_{2} \in \Pi$. Hence, the residual $E \Pi^{\prime}$ is of the lowest-priority. On the contrary, the lowest priority formulae could produce a loop. They are formulae $A \Pi$ where $\Pi$ contains at least one $\sigma_{1} \mathcal{U} \square \sigma_{2}$ or $\square\left(\sigma_{1} \mathcal{U} \sigma_{2}\right)$ and formulae $\mathrm{E} \Pi$ where $\Pi$ contains at least one $\square\left(\sigma_{1} \mathcal{U} \sigma_{2}\right)$ (but are 340 not of the highest priority). They could produces a loop in a finite number of steps, since the subformulae starting by $\square$ remains forever in the E-disjunctive formulae, whereas in the A-disjunctive formulae they can either remain or disappear. In the latter case, the residual A-disjunctive formulae could become non-selectable. It is easy to see that non-selectable formulae necessarily produce a loop.

Example 23. Consider $\Sigma_{0}=\{\mathrm{A}(a \mathcal{U} b, b \mathcal{U} c), \mathrm{E}(p \mathcal{U} q, \square(r \mathcal{U} s)), \mathrm{A} \square(c \mathcal{U} d), \neg b, \mathrm{~A} \square e\}$. The first two formulae have ${ }_{345}$ the highest-priority, the third has the lowest priority, and the the last two are non-selectable. Suppose that we select $\mathrm{A}(a \mathcal{U} b, b \mathcal{U} c)$ and mark $a \mathcal{U} b$, since $\neg b \in \Sigma_{0}$, the left-most open branch of rule $(\mathrm{A} \mathcal{U} \sigma)^{+}$contains $a, \mathrm{~A}(\circ((a \wedge$ $\left.\left.\left.\neg \Sigma_{0}^{\prime}\right) \mathcal{U} b\right), b \mathcal{U} c\right)$ where $\Sigma_{0}^{\prime}=\Sigma_{0} \backslash\{\mathrm{A}(a \mathcal{U} b, b \mathcal{U} c)\}$. After applying the corresponding $\alpha$ and $\beta$ rules to the remaining formulae, the first stage $s_{0}$ (the first state of the model) contains the atoms $\{a, q, s, d, e\}$. Then, by the next-step rule (Q०), the first node of the second stage $s_{1}$ is $\Sigma_{1}=\left\{\mathrm{A}\left(\left(a \wedge \neg \Sigma_{0}^{\prime}\right) \mathcal{U} b, b \mathcal{U} c\right), \mathrm{E} \square(r \mathcal{U} s), \mathrm{A} \square(c \mathcal{U} d), \mathrm{A} \square\right.$ e $\}$ where the

350 first formula is kept selected and the first eventuality is kept marked. Note that the second formula has now the lowest priority. Then we apply $(\mathrm{A} \mathcal{U} \sigma)^{+}$to the first formulae and the corresponding $\alpha$ and $\beta$ rules to the remaining formulae in $\Sigma_{1}$, generating the set of atoms in the left-most branch are $\{b, s, d, e\}$. Then, by the next-step rule (Q०), the first node of the third stage $s_{2}$ is $\Sigma_{2}=\{\mathrm{E} \square(r \mathcal{U} s), \mathrm{A} \square(c \mathcal{U} d), A \square e\}$ where the first two formulas are of the lowest priority and the third is non-selectable. By selecting the first formula, the atoms in the stages $s_{2}$ (of the left-most branch) are $355\{s, d, e\}$ and the new uniform set at the first node of the following stage is $\Sigma_{3}=\Sigma_{2}$. However, $\mathrm{A} \square(c \mathcal{U} d)$ has not been selected inside the loop, hence we produce one stage more, $s_{3}$, with atoms $\{s, d, e\}$. Then, by (Q०), we obtain $\Sigma_{4}=\Sigma_{2}$ and the branch is eventuality-covered. Therefore, we have a model for $\Sigma_{0}$ is $s_{0}, s_{1}\left\langle s_{2}, s_{3}\right\rangle^{\omega}$.

Definition 24 (Priorities for Eventuality Selection). The selectable formulae of the highest priority for Eventuality_Selection are the formulae of the following two forms:

- AП where $\Pi$ is exclusively formed by formulae of the form $\sigma_{1} \mathcal{U}\left(\sigma_{2} \wedge \diamond \sigma_{3}\right)$.

- $\mathrm{E} \Pi$ where $\Pi$ contains at least one eventuality of the form $\sigma_{1} \mathcal{U}\left(\sigma_{2} \wedge \diamond \sigma_{3}\right)$ or $\sigma_{1} \mathcal{U} \square \sigma_{2}$. 
Consequently, the (selectable) formulae of the lowest priority are the formulae of the following two forms:

- $\mathrm{A} \Pi$ where $\Pi$ contains at least one $\sigma_{1} \mathcal{U} \square \sigma_{2}$ or $\square\left(\sigma_{1} \mathcal{U} \sigma_{2}\right)$.

- EП where $\Pi$ does not contain any $\sigma_{1} \mathcal{U}\left(\sigma_{2} \wedge \diamond \sigma_{3}\right)$ nor $\sigma_{1} \mathcal{U} \square \sigma_{2}$, and $\Pi$ contains at least one $\square\left(\sigma_{1} \mathcal{U} \sigma_{2}\right)$.

Once all the highest priority formulae have been selected in a branch, the only selectable formulae are the lowest priority formulae. At this point, the objective is to get a loop-node that makes the branch eventuality-covered.

Definition 25 (Loop-node). Let $b$ be a tableau branch and $n_{i} \in b(0 \leq i)$. Then $n_{i}$ is a loop-node if there exists $n_{j} \in b(0 \leq j<i)$ and $\tau\left(n_{i}\right)=\tau\left(n_{j}\right)$. We say that $n_{j}$ is a companion node of $n_{i}$.

Definition 26 (Eventuality-covered Branch). A tableau branch $b=n_{0}, n_{1}, \ldots, n_{i}$ is eventuality-covered if $n_{i}$ is a loop-node, with a companion node $n_{j}(0 \leq j<i)$, both labelled by a uniform set $\Sigma$ of non-selectable and the lowest priority formulae such that every selectable formula $\mathrm{Q} \Pi \in \tau\left(n_{i}\right)$ is selected in some node $n_{k}(j \leq k<i)$ and for every selected AП exactly one eventuality in $\Pi$ is marked in some node $n_{k}$ such that $j \leq k<i$.

The procedure Eventuality_Selection performs in some fair way that ensures that any open branch will ever be eventuality-covered.

Definition 27 (Non-expandable Node). A node $n$ is non-expandable, if $\tau(n)=\Sigma_{\perp}$ or $n$ is a loop-node of branch $b$ which is eventuality-covered. Otherwise, $n$ is expandable.

Consequently, an expandable node is either a node that is not a loop-node or a loop-node whose branch is not eventuality-covered. It is worth noting that a formula of the lowest priority could be selected more than once in a branch because the loop-node could change along the branch. In the following Example 28, we illustrate this issue.

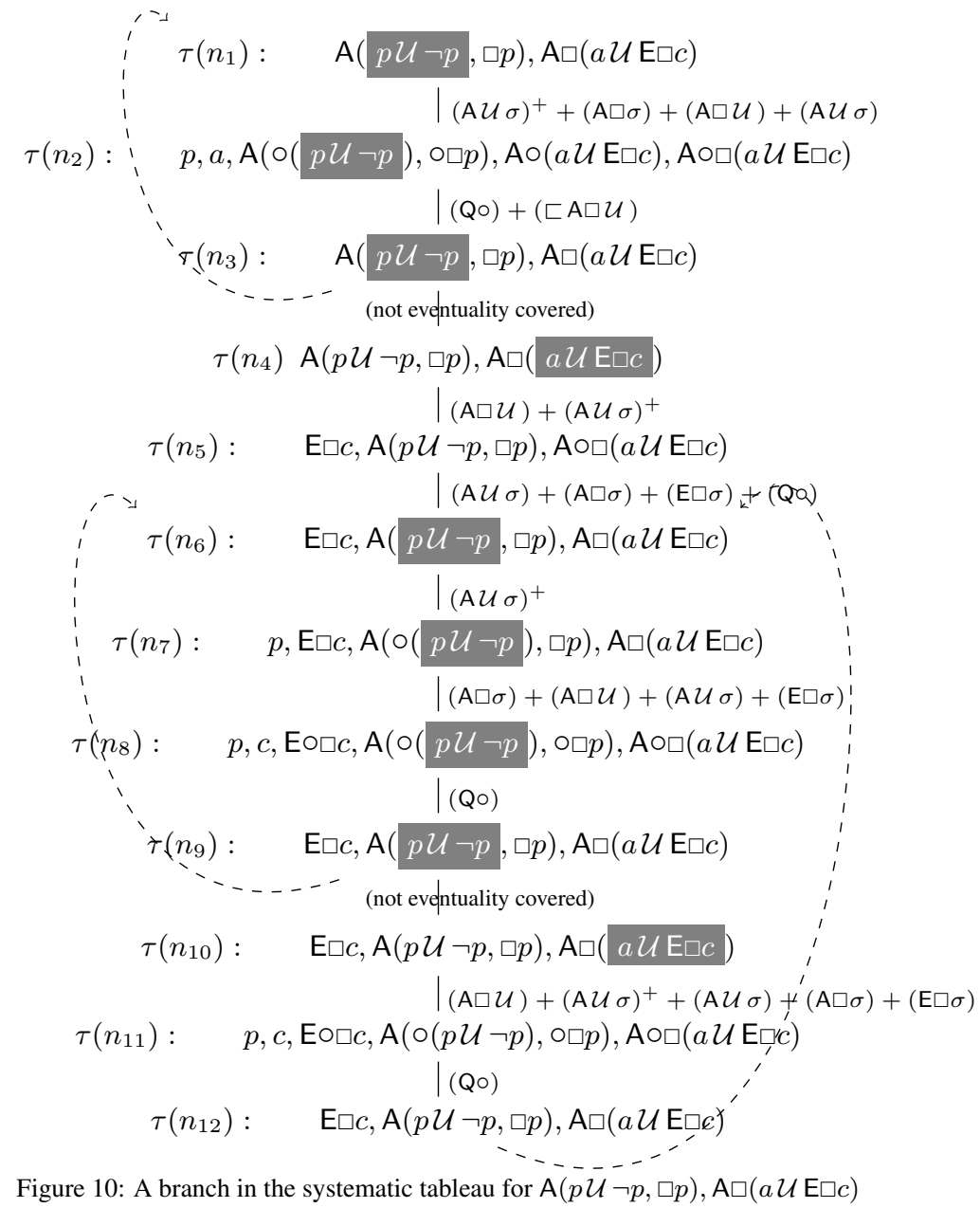


Example 28. Figure 10 shows a branch in the systematic tableau for $\Sigma_{0}=\{\mathrm{A}(p \mathcal{U} \neg p, \square p), \mathrm{A} \square(a \mathcal{U} \mathrm{E} \square c)\}$ where, for readability, the marked eventualities are in gray boxes. The call Eventuality_Selection $\left(\Sigma_{0}\right)$ selects the formula $\mathrm{A}(p \mathcal{U} \neg p, \square p)$ in $n_{1}$. Generating $n_{2}$, when we apply the $(\mathrm{A} \mathcal{U} \sigma)^{+}$rule to $\mathrm{A}(p \mathcal{U} \neg p, \square p)$, the inner context is $p$ and the outer context is $\mathrm{A} \square(a \mathcal{U} \mathrm{E} \square c)$. Hence, in the $S_{\Sigma, \beta_{2}}^{+}$branch, the next-step variant of $p \mathcal{U} \neg p$ is $p \wedge(\neg(\mathrm{A} \square(a \mathcal{U} \mathrm{E} \square c) \vee$ $p)) \mathcal{U} \neg p$. By classical subsumption (included in our simplification rules), $p \wedge(\neg(\mathrm{A} \square(a \mathcal{U} \mathrm{E} \square c) \vee p) \longrightarrow p$, hence the next-step variant is $p \mathcal{U} \neg p$, and the formula $\mathrm{A}(\circ(p \mathcal{U} \neg p), \square p)$ is added to the current stage. Then, applying $(\mathrm{A} \square \sigma)$ to $\mathrm{A}(\mathrm{\circ}(p \mathcal{U} \neg p), \square p),(\mathrm{A} \square \mathcal{U})$ to $\left(\mathrm{A} \square(a \mathcal{U} \mathrm{E} \square c)\right.$, and $(\mathrm{A} \mathcal{U} \sigma)$ to $\mathrm{A}(a \mathcal{U} \mathrm{E} \square c)$, the node $n_{2}$ is obtained. After the application of (Q०) and ( $\sqsubset \mathrm{A} \square \mathcal{U}), n_{3}$ is obtained. Node $n_{3}$ is a loop-node whose companion node is $n_{1}\left(\tau\left(n_{3}\right)=\tau\left(n_{1}\right)\right)$. However, the branch is not eventually-covered since the eventuality aU $\mathrm{E} \square c$ is not selected inside the loop. Therefore, we obtain $n_{4}$ by the call Eventuality_Selection $\left(\left(\tau\left(n_{3}\right)\right)\right.$ which selects $\mathrm{A}(a \mathcal{U} \mathrm{E} \square c)$. After applying the $(\mathrm{A} \mathcal{U} \sigma)^{+}$rule to $\mathrm{A}(a \mathcal{U} \mathrm{E} \square c)$ (once the $(\mathrm{A} \square \mathcal{U})$ rule is applied), the branch $S_{\Sigma, \beta_{1}}^{+}$expands to $n_{5}$. After that, Uniform_Tableau gets one expandable node labelled by the uniform set $\{\mathrm{E} \square c, \mathrm{~A}(p \mathcal{U} \neg p, \square p), \mathrm{A} \square($ a $\mathcal{U} \mathrm{E} \square c)\}$ as represented in $n_{6}$. The call Eventuality_Selection $\left(\left(\tau\left(n_{6}\right)\right)\right.$ selects again the formula $\mathrm{A}(p \mathcal{U} \neg p, \square p)$. Now, the inner context is $p$ and the outer context is $\{\mathrm{E} \square c, \mathrm{~A} \square(a \mathcal{U} \mathrm{E} \square c)\}$. Hence, by subsumption, $p \wedge(\neg(\mathrm{E} \square c) \vee \neg(\mathrm{A} \square(a \mathcal{U} \mathrm{E} \square c)) \vee p) \longrightarrow p$. Hence, the $S_{\Sigma, \beta_{2}}^{+}$ branch contains again the next-step variant $p \mathcal{U} \neg$ p in $n_{7}$. Then, expanding the Uniform_Tableau we obtain $n_{8}$ which is an expandable loop-node because $\tau\left(n_{8}\right)=\tau\left(n_{6}\right)$. However, the branch is not yet eventually-covered since aU $\mathrm{E} \square c$ has not been marked inside the loop. Then, the selected formula in $n_{9}$ is $\mathrm{A}(a \mathcal{U} \mathrm{E} \square c)$. Finally, Uniform_Tableau obtains a non-expandable loop-node, thus the given branch is eventually-covered-depicted in Figure 10 it represents $\{p, a\}(\{p, c\})^{w}$, which is a model of $\Sigma_{0}$. However, considering all the nodes in the branch, one would realize that the model represented is $\{p, a\}\{p, c\}(\{p, a, c\}\{p, c\})^{w}$.

Definition 29 (Bunch in a Tableau, Closed Bunch and Tableau). A bunch $b$ is a collection of branches that is maximal with respect to (Q०)-successor, i.e. every (Q०)-successor of any node in b is also in b. A bunch is closed iff at least one of its branches is closed, otherwise it is open. A tableau is closed iff all its bunches are closed.

Therefore, any open tableau has at least one open bunch, formed by one or more open branches. To complete, this section we provide two examples: a closed tableau and an open tableau. We mark eventualities in gray boxes and use large circles to represent the generation of AND-nodes or bunches.

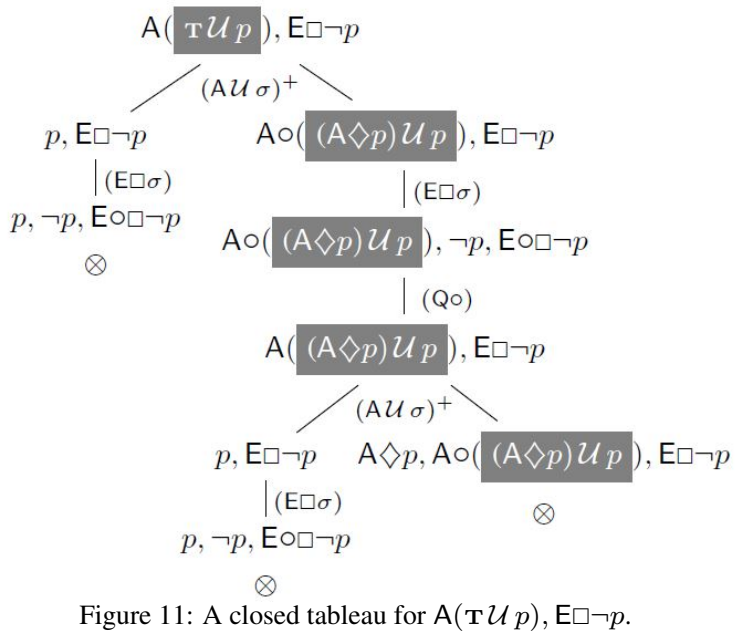

Example 30. Figure 11 shows a closed tableau for $\mathrm{A}(\mathrm{T} \mathcal{U} p), \mathrm{E} \square \neg p$. Note that, in the two applications of the rule $(\mathrm{A} \mathcal{U} \sigma)^{+}$, the inner context is empty and the outer context is $\mathrm{E} \square \neg p$ whose negation in nnf is $\mathrm{A} \diamond p$. Hence, the label of the rightmost node, $\mathrm{A} \circ((\mathrm{A} \diamond p) \mathcal{U} p)$, is the simplification of the selected formula $\mathrm{A} \circ(((\mathrm{A} \diamond p) \wedge(\mathrm{A} \diamond p)) \mathcal{U} p)$.

Example 31. On the left of Figure 12 we depict a representative open bunch of a tableau for the set of formulae: ${ }_{410} p, \mathrm{~A} \square(\mathrm{E} \circ p \wedge \mathrm{E} \circ \neg p), \mathrm{A}(\diamond \neg p, \square p)$. For saving space, we apply at once the Uniform_Tableau procedure subsequently choosing one of the leaves produced. Note that, for each node, we draw only one of the OR-children, but all the 


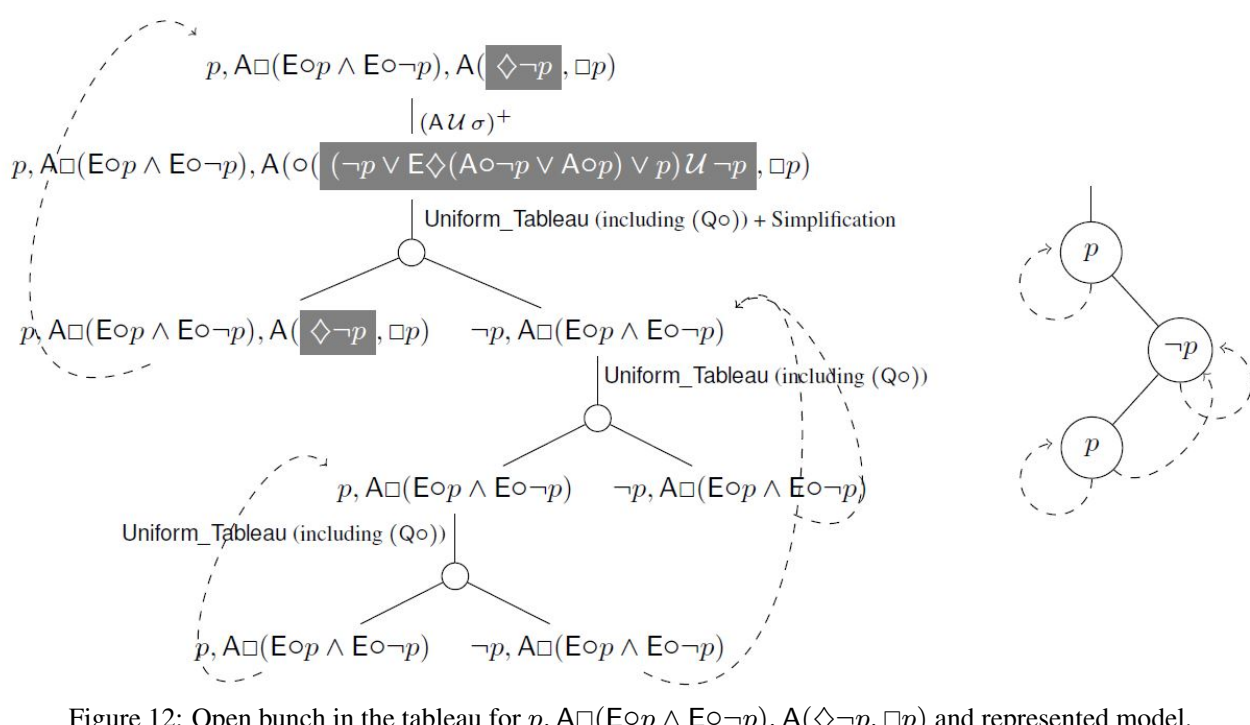

AND-children. In the marked eventuality, $\neg p \vee \mathrm{E} \vee(\mathrm{A} \circ \neg p \vee \mathrm{A} \circ p)$ comes from the negation of the outer context, and the disjunct $p$ from the inner context. By 'Simplification' $\neg p \vee \mathrm{E} \diamond(\mathrm{A} \circ \neg p \vee \mathrm{A} \circ p) \vee p$ is reduced to $\mathrm{T}$ (in the left-hand child). In the label of the right-hand node, $\neg p$ subsumes $\mathrm{A}((\ldots) \mathcal{U} \neg p, \square p)$. This open bunch represents a model (of the input set of formulae) that we depict on the right of Figure 12

\section{Soundness}

To prove the soundness of our tableau method (Theorem 34 we show that every tableau rule preserves satisfiability (Lemma 33). To prove the latter we essentially use the limit closure property, ensuring that the satisfiability of the negated inner context is preserved from segments of a limit path to the limit path itself (Proposition 32). The use of $\varphi_{\Pi}$ (Definition 19) is crucial here.

Proposition 32 (Preservation of the Negated Inner Context ). Let $\Pi$ be any set of basic path formulae and let $\varphi_{\Pi}$ be as in Definition 19 . Let $\mathcal{K}$ be a Kripke structure, $x_{1} \in$ fullpaths $(\mathcal{K})$ such that $\mathcal{K}, x 1,0 \models \neg \Pi$. Let $y=x_{1}^{\leq i_{1}} x_{2}^{\leq i_{2}} \cdots x_{k}^{\leq i_{k}} \cdots$ be a limit path in fullpaths $(\mathcal{K})$, for some $i_{1}>0$ and some $x_{2}^{\leq i_{2}} \cdots x_{k}^{\leq i_{k}} \cdots$. Then $\mathcal{K}, y, 0=\neg \pi$ holds for all $\pi \in \Pi$, provided that the following two conditions hold for all $n \geq 1$ :

(a) $\mathcal{K}, x_{1}^{\leq i_{1}} x_{2}^{\leq i_{2}} \cdots x_{n}, j \models \neg \sigma_{2}$ for all $\sigma_{1} \mathcal{U}\left(\sigma_{2} \wedge \diamond \sigma_{3}\right) \in \Pi$ and all $j \in\left\{0 . . i_{n}\right\}$, and

(b) $\mathcal{K}, x_{1}^{\leq i_{1}} x_{2}^{\leq i_{2}} \cdots x_{n}^{\leq i_{n}}, i_{n} \models \neg \varphi_{\Pi}$.

PROOF. We check the four cases of a basic path formula $\pi \in \Pi$. If $\pi$ is of the form $\circ \sigma$, then $\mathcal{K}, y, 0 \models \neg \circ \sigma$ because $\mathcal{K}, y, 0=\neg \pi$ and $i_{1}>0$. If $\pi$ is of the form $\sigma_{1} \mathcal{U}\left(\sigma_{2} \wedge \diamond \sigma_{3}\right)$, then property (a) ensures that every state in $y$ satisfies $\neg \sigma_{2}$. Therefore, $\neg\left(\sigma_{1} \mathcal{U}\left(\sigma_{2} \wedge \diamond \sigma_{3}\right)\right)$ is satisfied along the limit path $y$. For the remaining three cases, on the basis of (b) and Definition 19 we can prove the following three facts: (1) If $\pi=\square\left(\sigma_{1} \vee \square \sigma_{2}\right)$, then $\mathcal{K}, x_{1}^{\leq i_{1}} x_{2}^{\leq i_{2}} \cdots x_{n}^{\leq i_{n}}, i_{n} \models$ $\neg \sigma_{1} \wedge \neg \sigma_{2}$ for all $n$. (2) If $\pi=\square\left(\sigma_{1} \mathcal{U} \sigma_{2}\right)$, then we have that $\mathcal{K}, x_{1}^{\leq i_{1}} x_{2}^{\leq i_{2}} \cdots x_{n}^{\leq i_{n}}, i_{n} \models \neg \mathrm{E}\left(\diamond \sigma_{2}\right)$ for all $n$. (3) If $\pi=\sigma_{1} \mathcal{U} \square \sigma_{2}$, then $\mathcal{K}, x_{1}^{\leq i_{1}} x_{2}^{\leq i_{2}} \cdots x_{n}^{\leq i_{n}}, i_{n} \models \neg \sigma_{2}$ for all $n$. Therefore, in any of the three cases, we can ensure that $\mathcal{K}, y, 0 \models \pi$.

Lemma 33 (Soundness of the Tableau Rules). For any set of state formulae $\Sigma$ :

(i) For any $\alpha$-formula $\alpha$ : Sat $(\Sigma, \alpha)$ iff $\operatorname{Sat}\left(\Sigma, S_{\alpha}\right)$.

(ii) For any $\beta$-formula $\beta$ of range $k$ : Sat $(\Sigma, \beta)$ iff $\operatorname{Sat}\left(\Sigma, S_{\beta_{i}}\right)$ for some $1 \leq i \leq k$.

(iii) For any $\beta^{+}$-formula $\beta$ of range $k$ : Sat $(\Sigma, \beta)$ iff $\operatorname{Sat}\left(\Sigma, S_{\Sigma, \beta_{i}}^{+}\right)$for some $1 \leq i \leq k$.

(iv) If $\Sigma$ is a set of consistent literals: $\operatorname{Sat}\left(\Sigma, \mathrm{A} \circ \Phi_{1}, \ldots, \mathrm{A} \circ \Phi_{n}, \mathrm{E} \circ \Psi_{1}, \ldots, \mathrm{E} \circ \Psi_{m}\right)$ iff for all $0 \leq i \leq m$ : $\operatorname{Sat}\left(\mathrm{A} \Phi_{1}, \ldots, \mathrm{A} \Phi_{n}, \mathrm{E} \Psi_{i}\right)$. 
PROOF. Noting that (i), (ii) and (iv) can be easily proved by the 'systematic' application of the semantic definitions of temporal operators, we prove (iii). The 'only if' direction' for each of the cases of $\beta^{+}$-rules is trivial. We will prove the 'if' direction of the three rules $(\mathrm{E} \mathcal{U} \sigma \square)^{+},(\mathrm{A} \mathcal{U} \sigma)^{+}$and $(\mathrm{A} \mathcal{U} \square)^{+}$, in this order.

For the 'if' direction of rule $(\mathrm{E} \mathcal{U} \sigma \square)^{+}$, let us suppose that $\mathcal{K} \models \Sigma$, Eח, where $\Pi$ contains at least one eventuality. There exists $x \in$ fullpaths $(\mathcal{K})$ such that $\mathcal{K}, x, 0 \models \Sigma$, I. We are going to prove that there exists $\mathcal{K}^{\prime}$ such that one of he following properties holds:

(a) $\mathcal{K}^{\prime} \models \Sigma, \sigma_{2}, \mathrm{E}\left(\diamond \sigma_{3}, \Pi^{-i}\right)$ for some $\pi_{i}=\sigma_{1} \mathcal{U}\left(\sigma_{2} \wedge \nabla_{3}\right)$ in $\Pi_{\mathcal{U}}$

(b) $\mathcal{K}^{\prime} \models \Sigma, \mathrm{E}\left(\square \sigma_{2}, \Pi^{-i}\right)$ for some $\pi_{i}=\sigma_{1} \mathcal{U} \square \sigma_{2}$ in $\Pi_{\mathcal{U}}$

(c) $\mathcal{K}^{\prime}=\Sigma, \sigma_{1}, \mathrm{E}\left(\circ\left(\left(\sigma_{1} \wedge \neg \Sigma\right) \mathcal{U}\left(\sigma_{2} \wedge \diamond \sigma_{3}\right)\right), \Pi^{-i}\right)$ for some $\pi_{i}=\sigma_{1} \mathcal{U}\left(\sigma_{2} \wedge \diamond \sigma_{3}\right)$ in $\Pi_{\mathcal{U}}$

(d) $\mathcal{K}^{\prime}=\Sigma, \sigma_{1}, \mathrm{E}\left(\mathrm{O}\left(\left(\sigma_{1} \wedge \neg \Sigma\right) \mathcal{U} \square \sigma_{2}\right), \Pi^{-i}\right)$ for some $\pi_{i}=\sigma_{1} \mathcal{U} \square \sigma_{2}$ in $\Pi_{\mathcal{U}}$.

${ }_{450}$ Since $\mathcal{K}, x, 0=\pi_{i}$ for all $\pi \in \Pi_{\mathcal{U}}$, we define $j$ to be the least $i \geq 0$ such that $\mathcal{K}, x, i=\varphi$ for some $\sigma_{1} \mathcal{U} \varphi \in \Pi_{\mathcal{U}}$. If $j=0$, then (a) and (b) (depending on the form of $\varphi$ ) are trivially satisfied for $\mathcal{K}^{\prime}=\mathcal{K}$. Otherwise, if $j>0$, it holds that $\mathcal{K}, x, j \models \varphi$ and for all $i<j: \mathcal{K}, x, i \models \sigma_{1}$. Moreover, as $j$ is the minimal index, for all $0 \leq i<j: \mathcal{K}, x, i \models \sigma_{1}^{\prime}$ for all $\sigma_{1}^{\prime} \mathcal{U} \varphi^{\prime} \in \Pi_{\mathcal{U}}$. Consider $k$ to be the greatest index $i$ such that $0 \leq i<j$ and $\mathcal{K}, x, i \models \Sigma$. Henceforth, we have that $\mathcal{K}, x, k \models \Sigma$ and $\mathcal{K}, x, h \models \neg \Sigma$, for all $h$ such that $k+1 \leq h<j$. Therefore, (c) and (d) hold for $\mathcal{K}^{\prime}=\mathcal{K}\lceil x(k)$.

For the 'if' direction of the rule $(\mathrm{A} \mathcal{U} \sigma)^{+}$, let us suppose that the three sets $\Sigma \cup S_{\Sigma, \beta_{1}}, \Sigma \cup S_{\Sigma, \beta_{2}}$, and $\Sigma \cup S_{\Sigma, \beta_{3}}$ of the rule $(\mathrm{A} \mathcal{U} \sigma)^{+}$are unsatisfiable. We will show that the set $\Sigma, \mathrm{A}\left(\sigma_{1} \mathcal{U}\left(\sigma_{2} \wedge \diamond \sigma_{3}\right), \Pi\right)$ must be also unsatisfiable. By the hypothesis, we know that any model of $\Sigma$ is not a model of $S_{\Sigma, \beta_{i}}$ for all $i \in\{1,2,3\}$. In other words, for any $\mathcal{K}$ such that $\mathcal{K} \models \Sigma$, the followings three facts holds:

(a) $\mathcal{K} \not \sigma_{2} \wedge \mathrm{A}\left(\diamond \sigma_{3}, \Pi\right)$

${ }_{460} \quad$ (b) $\mathcal{K} \not \neq \sigma_{1} \wedge \mathrm{A}\left(\circ\left(\left(\sigma_{1} \wedge\left(\neg \Sigma \vee \varphi_{\Pi}\right)\right) \mathcal{U}\left(\sigma_{2} \wedge \diamond \sigma_{3}\right)\right), \Pi\right)$

(c) $\mathcal{K} \not=A \Pi$

To show that $\Sigma, \mathrm{A}\left(\sigma_{1} \mathcal{U}\left(\sigma_{2} \wedge \diamond \sigma_{3}\right), \Pi\right)$ is unsatisfiable, we consider an arbitrary $\mathcal{K}$ such that $\mathcal{K} \models \Sigma$ and prove that $\mathcal{K} \not \models \mathrm{A}\left(\sigma_{1} \mathcal{U}\left(\sigma_{2} \wedge \diamond \sigma_{3}\right), \Pi\right)$. Since $\mathcal{K} \models \Sigma$, then (a), (b) and (c) hold. According to (b), there are two possible cases: (Case 1): If $\mathcal{K} \not \models \sigma_{1}$ then, by (a), either $\mathcal{K} \models \neg \sigma_{1} \wedge \neg \sigma_{2}$ or $\mathcal{K} \models \neg \sigma_{1} \wedge \mathrm{E}\left(\square \neg \sigma_{3}, \neg \Pi\right)$. In both cases, it is easy to see 465 that $\mathcal{K} \not=\mathrm{A}\left(\sigma_{1} \mathcal{U}\left(\sigma_{2} \wedge \diamond \sigma_{3}\right), \Pi\right)$.

(Case 2): Otherwise, if $\mathcal{K} \not \models \mathrm{A}\left(\circ\left(\left(\sigma_{1} \wedge\left(\neg \Sigma \vee \varphi_{\Pi}\right)\right) \mathcal{U}\left(\sigma_{2} \wedge \diamond \sigma_{3}\right)\right)\right.$, $\left.\Pi\right)$, then there exists $x_{1} \in$ fullpaths $(\mathcal{K})$ such that $\mathcal{K}, x_{1}, 0 \models \circ \neg\left(\left(\sigma_{1} \wedge\left(\neg \Sigma \vee \varphi_{\Pi}\right)\right) \mathcal{U}\left(\sigma_{2} \wedge \diamond \sigma_{3}\right)\right) \wedge \neg \Pi$. This yields two possible cases:

(Case 2.1): If $\mathcal{K}, x_{1}, 0 \models \circ \square\left(\neg \sigma_{2} \vee \square \neg \sigma_{3}\right) \wedge \neg \Pi$, then it is trivial that $\mathcal{K} \forall \forall \mathrm{A}\left(\sigma_{1} \mathcal{U}\left(\sigma_{2} \wedge \diamond \sigma_{3}\right), \Pi\right)$.

(Case 2.2): Otherwise, there should exist $i_{1}>0$ that satisfies the following three properties:

$470 \quad$ (i) $\mathcal{K}, x_{1}, j \models\left(\neg \sigma_{2}\right) \vee \square \neg \sigma_{3}$ for all $j$ such that $0 \leq j \leq i_{1}$, and

(ii) $\mathcal{K}, x_{1}, i_{1} \models \neg \sigma_{1} \vee\left(\Sigma \wedge \neg \varphi_{\Pi}\right)$, and

(iii) $\mathcal{K}, x_{1}, 0 \models \neg \Pi$

If (i) is satisfied because $\mathcal{K}, x_{1}, j \models \square \neg \sigma_{3}$ (for some $j$ such that $0 \leq j \leq i_{1}$ ) then trivially $\mathcal{K}, x_{1}, 0 \not \models \sigma_{1} \mathcal{U}\left(\sigma_{2} \wedge \triangleright \sigma_{3}\right)$. This, along with the fact (iii), not only ensures that $\mathcal{K} \forall \mathrm{A}\left(\sigma_{1} \mathcal{U}\left(\sigma_{2} \wedge \diamond \sigma_{3}\right), \Pi\right)$ but also applies to any other formula ${ }_{475} \sigma_{1}^{\prime} \mathcal{U}\left(\sigma_{2}^{\prime} \wedge \diamond \sigma_{3}^{\prime}\right)$ in $\Pi$. Henceforth, in what follows, we can suppose that for all $j$ such that $0 \leq j \leq i_{1}: \mathcal{K}, x_{1}, j \models \neg \sigma_{2}$ and also that $\mathcal{K}, x_{1}, j \models \neg \sigma_{2}^{\prime}$ for all $\sigma_{1}^{\prime} \mathcal{U}\left(\sigma_{2}^{\prime} \wedge \diamond \sigma_{3}^{\prime}\right) \in \Pi$.

If (ii) is satisfied because $\mathcal{K}, x_{1}, i_{1} \models \neg \sigma_{1}$ then it is clear that $\mathcal{K}, x_{1}, 0 \not \models \sigma_{1} \mathcal{U}\left(\sigma_{2} \wedge \diamond \sigma_{3}\right)$. Therefore, by (i) and (iii), $\mathcal{K} \not=\mathrm{A}\left(\sigma_{1} \mathcal{U}\left(\sigma_{2} \wedge \diamond \sigma_{3}\right), \Pi\right)$.

Otherwise, if (ii) is satisfied because $\mathcal{K}, x_{1}, i_{1} \models \Sigma \wedge \neg \varphi_{\Pi}$, then (a), (b) and (c) hold for $\mathcal{K} \uparrow x_{1}\left(i_{1}\right)$ (instead of $\mathcal{K}$ ) 480 because $\mathcal{K}, x_{1}, i_{1} \models \Sigma$. Hence, applying the same reasoning for $\mathcal{K} \uparrow x_{1}\left(i_{1}\right)$ as we did above for $\mathcal{K}$, we conclude that there should be a path $x_{2} \in$ fullpaths $\left(\mathcal{K} \uparrow x_{1}\left(i_{1}\right)\right)$ such that one of the following two facts holds:

(Case 2.2.1): $\mathcal{K}\left\lceil x_{1}\left(i_{1}\right), x_{2} \models \square \neg\left(\sigma_{2} \wedge \diamond \sigma_{3}\right) \wedge \neg \Pi\right.$, and therefore $\mathcal{K} \not \models \mathrm{A}\left(\sigma_{1} \mathcal{U}\left(\sigma_{2} \wedge \diamond \sigma_{3}\right), \Pi\right)$.

(Case 2.2.2): there should exist $i_{2}>0$ such that $\mathcal{K}\left\lceil x_{1}\left(i_{1}\right), x_{2}, i_{2} \models \Sigma \wedge \neg \varphi_{\Pi}\right.$ and for all $j \in\left\{0 . . i_{2}\right\}$ :

- $\mathcal{K}\left\lceil x_{1}\left(i_{1}\right), x_{2}, j=\neg \sigma_{2}\right.$, and

- $\mathcal{K}\left\lceil x_{1}\left(i_{1}\right), x_{2}, j \models \neg \sigma_{2}^{\prime}\right.$ for all $\sigma_{1}^{\prime} \mathcal{U}\left(\sigma_{2}^{\prime} \wedge \diamond \sigma_{3}^{\prime}\right) \in \Pi$

Now, (a), (b) and (c) apply to $\mathcal{K} \uparrow x_{2}\left(i_{2}\right)$. Hence, the infinite iteration of the second case yields a path $y=$ $x_{1}^{\leq i_{1}} x_{2}^{\leq i_{2}} \cdots x_{k}^{\leq i_{k}} \cdots$ (that exists by the limit closure property) for which the Proposition 32 ensures that $\mathcal{K}, y, 0 \not \forall$ $\mathrm{A}\left(\sigma_{1} \mathcal{U}\left(\sigma_{2} \wedge \diamond \sigma_{3}\right), \Pi\right)$.

The proof for the $(\mathrm{A} \mathcal{U} \square)^{+}$rule follows the same scheme. 
Theorem 34 (Soundness of the Tableau Method). Given any set of state formulae $\Sigma$, if there exists a closed tableau for $\Sigma$ then UnSat $(\Sigma)$.

Proof. Let $T_{\Sigma}$ be a closed tableau for $\Sigma$. The set of formulae labelling at least one leaf in each bunch is inconsistent and therefore unsatisfiable. Then, by Lemma 33 , the labelling of the root node, $\Sigma$, is unsatisfiable.

\section{Completeness}

In this section, we prove the completeness of the introduced tableau method. First, we define the notions of stage, expanded bunch, and expanded tableau. Then we prove the refutational completeness: every unsatisfiable set of state formulae has a closed tableau. In fact, we are going to prove that, for any set $\Sigma_{0}$, if the systematic tableau $\mathcal{A}_{\Sigma_{0}}^{\text {sys }}$, given by Algorithm 1 is open, then $\Sigma_{0}$ is satisfiable. That is, $\mathcal{A}_{\Sigma_{0}}^{\text {sys }}$ has at least one open bunch that allows us to construct a model of $\Sigma_{0}$. The final step of proving the completeness of the tableau method is establishing its termination.

\subsection{Open Bunch Model Construction}

In this subsection, we define a method to associate a Kripke structure to any open bunch of the systematic tableau $\mathcal{A}_{\Sigma_{0}}^{\text {sys }}$. Later, we prove that this Kripke structure is a model of $\Sigma_{0}$.

Definition 35 (Stage). Given a branch, b of a tableaux $T$, a stage in $T$ is every maximal subsequence of successive nodes $n_{i}, n_{i+1}, \ldots, n_{j}$ in $b$ such that $\tau\left(n_{k}\right)$ is not a $(\mathrm{Q} \circ)$-child of $\tau\left(n_{k-1}\right)$, for all $k$ such that $i<k \leq j$. We denote by stages $(b)$ the sequence of all stages of $b$. The successor relation on stages $(b)$ is induced by the successor relation on $b$. The labelling function $\tau$ is extended to stages as the union of the original $\tau$ applied to every node in a stage.

We prove that any open bunch of the systematic tableau $\mathcal{A}_{\Sigma_{0}}^{\text {sys }}$ represents a model of the initial set of formulae $\Sigma_{0}$.

Definition $36\left(\alpha \beta^{+}\right.$-saturated Stage). A set of state formulae $\Psi$ is $\alpha \beta^{+}$-saturated iff for all $\sigma \in \Psi$ :

1. If $\sigma$ is an $\alpha$-formula then $S_{\sigma} \subseteq \Psi$

2. If $\sigma$ is a $\beta$-formula of range $k$, but it is not a $\beta^{+}$-formula, then $S_{\beta_{i}} \subseteq \Psi$ for some $1 \leq i \leq k$.

3. If $\sigma$ is a $\beta$-formula and also a $\beta^{+}$-formula of range $k$ then either $S_{\beta_{i}} \subseteq \Psi$ or $S_{\Sigma, \beta_{i}}^{+} \subseteq \Psi$ for some $1 \leq i \leq k$ and $\Sigma=\tau(n) \backslash\{\sigma\}$ for some $n \in s$.

We say that a stage $s$ in $\mathcal{A}_{\Sigma_{0}}^{\text {sys }}$ is $\alpha \beta^{+}$-saturated iff $\tau(s)$ is $\alpha \beta^{+}$-saturated. For a given set $\Sigma$ of state-formulae, we denote by $\operatorname{Comp}(\Sigma)$ the union of all the minimal sets that contains $\Sigma$ and are $\alpha \beta^{+}$-saturated.

Definition 37 (Expanded Bunch and Tableau). An open branch b is expanded if each stage $s \in \operatorname{stages}(b)$ is $\alpha \beta^{+}$. saturated and $b$ is eventuality-covered. A bunch is expanded if all its open branches are expanded. A tableau is expanded if all its open bunches are expanded.

Proposition 38. Given any set of state formulae $\Sigma_{0}$, the systematic tableau $\mathcal{A}_{\Sigma_{0}}^{\text {sys }}$ is expanded.

PROOF. Trivial, by construction.

Definition 39 (Open Bunch Model Construction). For any expanded bunch $H$ of $\mathcal{A}_{\Sigma_{0}}^{\text {sys }}$, we define the Kripke-structure $\mathcal{K}_{H}=(S, R, L)$ such that $S=\bigcup_{b \in H}$ stages $(b)$ and for any $s \in S: L(s)=\{p \mid p \in \tau(n) \cap$ Prop for some node $n \in$ $s\}$; and $R$ is the relation induced in $\operatorname{stages}(b)$ for each $b \in H$.

\subsection{Properties of the Open Branches of $\mathcal{A}_{\Sigma_{0}}^{\text {sys }}$}

In order to prove that $\mathcal{K}_{H}$, as defined in Definition 39 is a model for the label of the root of $H$, we first prove the required auxiliary properties of the systematic construction of the tableau $\mathcal{A}_{\Sigma_{0}}^{\text {sys }}$. The systematic construction of Algorithm 1 produces uniform sets as expandable nodes. The selection is always made in uniform sets and loop-nodes are also labelled by uniform sets. 
Remark 40 (Notation for Eventualities and Tableau Rule Application). In what follows, we use $\chi$ to represent a formula of one of the two following forms: $\left(\sigma_{2} \wedge \diamond \sigma_{3}\right)$ or $\square \sigma_{2}$. Then, $\sigma_{1} \mathcal{U} \chi$ stands for one of the two possible ${ }_{530}$ eventualities. We say that the corresponding $\beta^{+}$rule is applied to a selected $\mathrm{A} \Pi$ with some marked eventuality $\pi \in \Pi$, meaning that $(\mathrm{A} \mathcal{U} \sigma)^{+}$is applied when $\pi$ is $\sigma_{1} \mathcal{U}\left(\sigma_{2} \wedge \diamond \sigma_{3}\right),(\mathrm{A} \mathcal{U} \square)^{+}$is applied when $\pi$ is $\sigma_{1} \mathcal{U} \square \sigma_{2}$, and $(\mathrm{A} \square \mathcal{U})$ followed by $(\mathrm{A} \mathcal{U} \sigma)^{+}$is applied when $\pi$ is $\square\left(\sigma_{1} \mathcal{U} \sigma_{2}\right)$. We say that the corresponding $\beta^{+}$rule is applied to a selected $\mathrm{E} \Pi$ meaning that $(\mathrm{E} \mathcal{U} \sigma \square)^{+}$is applied when $\Pi$ contains at least one $\sigma_{1} \mathcal{U}\left(\sigma_{2} \wedge \diamond \sigma_{3}\right)$ or at lest one $\sigma_{1} \mathcal{U} \square \sigma_{2}$, and otherwise when $\Pi$ contains at least one $\square\left(\sigma_{1} \mathcal{U} \sigma_{2}\right)$, then $(\mathrm{E} \mathcal{U} \sigma \square)^{+}$is applied just after $(\mathrm{E} \square \mathcal{U})$ has been applied ${ }_{535}$ to every $\square\left(\sigma_{1} \mathcal{U} \sigma_{2}\right)$ in $\Pi$. For clarity, we consider sets $S_{\Sigma, \beta_{i}}$ and $S_{\Sigma, \beta_{i}}^{+}$used in the tableau rules creating child nodes from left $(i=1)$ to right, where $i$ is the rank of the rule.

Definition 41 (Variants). For a given set $\Pi$ of basic path formulae, we denote by Variants(П) the collection of all subsets of the sets $\Pi^{\prime}$ that are obtained from $\Pi$ by one simultaneous application of any number (including zero) of individual substitutions of some $\pi \in \Pi$ by $\pi^{\prime}$ that satisfies the following rules:

- $\pi$ is an eventuality $\sigma_{1} \mathcal{U}\left(\sigma_{2} \wedge \diamond \sigma_{3}\right)$ and $\pi^{\prime}$ is either $\diamond \sigma_{3}$ or a next-step variant of $\pi$.

- $\pi$ is an eventuality $\sigma_{1} \mathcal{U} \square \sigma_{2}$ and $\pi^{\prime}$ is either $\square \sigma_{2}$ or a next-step variant of $\pi$.

- $\pi$ is $\square\left(\sigma_{1} \vee \square \sigma_{2}\right)$ and $\pi^{\prime}$ is $\square \sigma_{2}$.

The following two propositions establish general properties of the rule-based decomposition of E-conjunctive and A-disjunctive formulae (respectively) along open branches.

Proposition 42. Let b be an open branch of $\mathcal{A}_{\Sigma_{0}}^{\text {sys }}$, let $s_{i}, s_{j}(i<j)$ be any pair of consecutive stages in stages $(b)$ and let $\Sigma \cup\{\mathrm{E \Pi}\}$ be the uniform set labelling the first node of $s_{i}$. There exists a (possibly empty and minimal) uniform set $\Pi^{\prime} \in \operatorname{Variants}(\Pi)$ such that $\mathrm{E}^{\prime} \in \tau\left(s_{j}\right)$ and for all $\pi \in \Pi$ :

(a) if $\pi=\sigma_{1} \mathcal{U}\left(\sigma_{2} \wedge \diamond \sigma_{3}\right)$ then there exists $k \geq i$ such that $\sigma_{1} \in \tau\left(s_{h}\right)$ for all $h \in i . . k-1$ and (al) $k<j$ and $\sigma_{2} \in \tau\left(s_{k}\right)$ and $\sigma_{3} \in \tau\left(s_{k^{\prime}}\right)$ for some $k^{\prime}$ such that $k \leq k^{\prime} \leq j$, or

(a2) $k<j$ and $\sigma_{2} \in \tau\left(s_{k}\right)$ and $\diamond \sigma_{3} \in \Pi^{\prime}$, or

(a3) $k=j$ and $\pi$ or some next-step variant of it is in $\Pi^{\prime}$.

(b) if $\pi=\sigma_{1} \mathcal{U} \square \sigma_{2}$ then there exists $k \geq i$ such that $\sigma_{1} \in \tau\left(s_{h}\right)$ for all $h \in i . . k-1$ and

(b1) $k<j$ and $\sigma_{2} \in \tau\left(s_{h}\right)$ for some $h$ such that $i \leq h \leq k$ and $\square \sigma_{2} \in \Pi^{\prime}$, or

(b2) $k=j$ and $\sigma_{1} \mathcal{U} \square \sigma_{2}$ or some next-step variant of it is in $\Pi^{\prime}$.

(c) if $\pi=\square\left(\sigma_{1} \mathcal{U} \sigma_{2}\right)$ then $\square\left(\sigma_{1} \mathcal{U} \sigma_{2}\right) \in \Pi^{\prime}$ and for all $h \in i . . j-1: \sigma_{2} \in \tau\left(s_{h}\right)$ or $\sigma_{1} \in \tau\left(s_{h}\right)$.

(d) if $\pi=\square\left(\sigma_{1} \vee \square \sigma_{2}\right)$ then there exists $k \geq i$ such that $\sigma_{1} \in \tau\left(s_{h}\right)$ for all $h \in i . . k-1$

(dl) $k=j$ and $\square\left(\sigma_{1} \vee \square \sigma_{2}\right) \in \Pi^{\prime}$, or

(d2) $k<j$ and there exists $k^{\prime} \in k . . j-1$ such that $\sigma_{2} \in \tau\left(s_{h}\right)$ for all $h \in k^{\prime} . . j-1$ and $\square \sigma_{2} \in \Pi^{\prime}$

Proof. By simultaneous induction on $\Pi$, using the rules for the E-conjunctive formulae. Note that any stage in $b$ is $\alpha \beta^{+}$-saturated and the procedure Uniform_Tableau applies exactly once between the last state of $s_{i}$ and the first state of $s_{i+1}$. More specifically, in (a) we use $(\mathrm{E} \overline{\mathcal{U}} \sigma)$ and $(\mathrm{E} \mathcal{U} \sigma \square)^{+}$, and (a1) and (a2) come from the second application of $(\mathrm{E} \mathcal{U} \sigma)$ to $\mathrm{E}\left(\diamond \sigma_{3}, \ldots\right)$ where $\diamond \sigma_{3}$ abbreviates $\mathbf{T} \mathcal{U} \sigma_{3}$. Similarly, item (b) comes from rules $(\mathrm{E} \mathcal{U} \square)$ and $(\mathrm{E} \mathcal{U} \sigma \square)^{+}$; Items (c) and (d) are respectively obtained from the rules $(\mathrm{E} \square \mathcal{U}),(\mathrm{E} \mathcal{U} \sigma)$, and $(\mathrm{E} \mathcal{U} \sigma \square)^{+}$; and $(\mathrm{E} \square \sigma)$. It is worth noting that $\Pi^{\prime}$ is empty if all the formulae in $\Pi$ satisfy the case (a1).

Proposition 43. Let $b$ be an open branch of $\mathcal{A}_{\Sigma_{0}}^{\text {sys }}$, let $s_{i}, s_{j}(j>i)$ be any pair of consecutive stages in $\operatorname{stages}(b)$ and let $\Sigma \cup\{\mathrm{A} \Pi\}$ be the uniform set labelling the first node of $s_{i}$. If there exists a non-empty uniform set $\Pi^{\prime} \in V^{\prime}$ ariants $(\Pi)$ such that $\mathrm{A \Pi}^{\prime} \in \tau\left(s_{j}\right)$, then the following four facts hold:

(a) For all $\sigma_{1} \mathcal{U}\left(\sigma_{2} \wedge \diamond \sigma_{3}\right) \in \Pi$ :

(al) if $\sigma_{1} \mathcal{U}\left(\sigma_{2} \wedge \diamond \sigma_{3}\right)$ or a next-step variant of it is in $\Pi^{\prime}$, then $\sigma_{1} \in \tau\left(s_{h}\right)$ for all $h \in i . . j-1$,

(a2) if $\diamond \sigma_{3} \in \Pi^{\prime}$ then $\sigma_{2} \in \tau\left(s_{k}\right)$ for some $k \in i . . j-1$ and $\sigma_{1} \in \tau\left(s_{h}\right)$ for all $h \in i . . k-1$.

(b) For all $\sigma_{1} \mathcal{U} \square \sigma_{2} \in \Pi$ : if $\square \sigma_{2} \in \Pi^{\prime}$ or $\sigma_{1} \mathcal{U} \square \sigma_{2}$ or a next-step variant of $\sigma_{1} \mathcal{U} \square \sigma_{2}$ is in $\Pi^{\prime}$, then $\left\{\sigma_{1}, \sigma_{2}\right\} \subseteq$ $\tau\left(s_{h}\right)$ for all $h \in i . . j-1$.

(c) For all $\square\left(\sigma_{1} \mathcal{U} \sigma_{2}\right) \in \Pi \cap \Pi^{\prime}: \sigma_{1} \in \tau\left(s_{h}\right)$ or $\sigma_{2} \in \tau\left(s_{h}\right)$ for all $h \in i . . j-1$.

(d) For all $\square\left(\sigma_{1} \vee \square \sigma_{2}\right) \in \Pi$, (d1) if $\square\left(\sigma_{1} \vee \square \sigma_{2}\right) \in \Pi$ then $\sigma_{1} \in \tau\left(s_{h}\right)$ for all $h \in i . . j-1$. 
(d2) if $\square \sigma_{2} \in \Pi^{\prime}$ then for some $k \in i . . j-1\left\{\neg \sigma_{1}, \sigma_{2}\right\} \subseteq \tau\left(s_{k}\right)$ and $\sigma_{1} \in \tau\left(s_{h}\right)$ for all $h \in i . . k-1$ and $\sigma_{2} \in \tau\left(s_{h}\right)$ for all $h \in k . . j-1$.

Moreover, if there exists no $\mathrm{A}^{\prime} \in \tau\left(s_{j}\right)$ such that $\Pi^{\prime} \in \operatorname{Variants}(\Pi)$, then there exists some $\sigma_{1} \mathcal{U}\left(\sigma_{2} \wedge \diamond \sigma_{3}\right) \in \Pi$ and some $k, k^{\prime} \in i . . j-1$ such that $k \leq k^{\prime}$ and $\sigma_{2} \in \tau\left(s_{k}\right)$ and $\sigma_{3} \in \tau\left(s_{k^{\prime}}\right)$.

580 PROOF. By simultaneous induction on $\Pi$. For the proof we note that any stage in $b$ is $\alpha \beta^{+}$-saturated, and the following rules for A-disjunctive formulae hold: $(\mathrm{A} \mathcal{U} \sigma)$ and $(\mathrm{A} \mathcal{U} \sigma)^{+}$in $(\mathrm{a}) ;(\mathrm{A} \mathcal{U} \square)$ and $(\mathrm{A} \mathcal{U} \square)^{+}$in $(\mathrm{b}) ;(\mathrm{A} \square \mathcal{U}),(\mathrm{A} \mathcal{U} \sigma)$, and $(\mathrm{A} \mathcal{U} \sigma)^{+}$in $(\mathrm{c})$; and $(\mathrm{A} \square \sigma)$ in $(\mathrm{d})$. It is worth noting that the rules $(\mathrm{A} \mathcal{U} \sigma)$ and $(\mathrm{A} \square \sigma)$ generate a child where the A-disjunctive formula that comes from $A \Pi$ is of the form $A \Pi^{\prime}$ with $\Pi^{\prime} \subset \Pi$ (one formula is lost).

From the previous two propositions it is easy to conclude that, for a given initial node (of stage $s_{i}$ ) labelled by a uniform set $\Sigma \cup\{\mathrm{Q} \Pi\}$, the mentioned sets $\Pi^{\prime}$ such that $\mathrm{Q} \Pi^{\prime} \in \tau\left(s_{i+1}\right)$ exclusively consist of path subformulae and next-step variants of the formulas in $\Pi$. It also follows that $\tau\left(s_{i+1}\right)$ exclusively contains Boolean combinations of state subformulae in $\Sigma \cup\{Q \Pi\}$ and the formula $Q \Pi^{\prime}$. The next proposition shows that the selection of an E-conjunctive formula in any open branch of $\mathcal{A}_{\Sigma_{0}}^{\text {sys }}$ can only be kept along finitely many stages.

Proposition 44. Let $b$ be an open branch of $\mathcal{A}_{\Sigma_{0}}^{\text {sys }}$, let $s_{i} \in \operatorname{stages}(b)$ and let $\Sigma \cup\{\mathrm{E \Pi}\}$ be the uniform set labelling 590 the first node of $s_{i}$ where the selectable formula $\mathrm{E} \Pi$ is selected. If $\mathrm{E} \Pi$ has the highest priority, then

(a) For all $\pi=\sigma_{1} \mathcal{U}\left(\sigma_{2} \wedge \diamond \sigma_{3}\right) \in \Pi:\left\{\sigma_{2}, \mathrm{E}\left(\diamond \sigma_{3}, \Pi^{\prime}\right)\right\} \subseteq \tau\left(s_{k}\right)$ for some stage $s_{k} \in \operatorname{stages}(b)$ ( $\left.k \geq i\right)$ and some $\Pi^{\prime} \in \operatorname{Variants}(\Pi \backslash\{\pi\})$.

(b) For all $\pi=\sigma_{1} \mathcal{U} \square \sigma_{2} \in \Pi: \mathrm{E}\left(\square \sigma_{2}, \Pi^{\prime}\right) \in \tau\left(s_{k}\right)$ for some stage $s_{k} \in \operatorname{stages}(b)(k \geq i)$ and some $\Pi^{\prime} \in$ Variants $(\Pi \backslash\{\pi\})$.

595 and if $\mathrm{E} \Pi$ has the lowest priority, then

(c) For all $\pi=\square\left(\sigma_{1} \mathcal{U} \sigma_{2}\right) \in \Pi: \sigma_{2} \in \tau\left(s_{k}\right)$ for some stage $s_{k} \in \operatorname{stages}(b)(k \geq i)$.

Proof. Suppose that EI has the highest priority, then $\Pi$ contains at least one $\sigma_{1} \mathcal{U}\left(\sigma_{2} \wedge \diamond \sigma_{3}\right)$ or $\sigma_{1} \mathcal{U} \square \sigma_{2}$, then the $\beta^{+}$-rule $(\mathrm{E} \mathcal{U} \sigma \square)^{+}$is applied in the first node of stage $s_{i}$ where $\mathrm{E} \Pi$ is selected and the resulting $\mathrm{E} \Pi^{\prime}$ of the highest priority in its children are kept selected, hence $(\mathrm{E} \mathcal{U} \sigma \square)^{+}$is again applied to them, and so on. We proceed by contradiction, supposing that (a) and (b) does not hold. We get a contradiction from the hypothesis that (a) does not hold, i.e. for some $\sigma_{1} \mathcal{U}\left(\sigma_{2} \wedge \diamond \sigma_{3}\right) \in \Pi:\left\{\sigma_{2}, \mathrm{E}\left(\diamond \sigma_{3}, \Pi^{\prime}\right)\right\} \nsubseteq \tau \tau\left(s_{k}\right)$ for every stage $s_{k} \in \operatorname{stages}(b)$ such that $k \geq i$ and all $\Pi^{\prime} \in \operatorname{Variants}\left(\Pi \backslash\left\{\pi_{i}\right\}\right)$. The proof for the case where (b) does not hold is identical. The fact that (a) does not hold means that at the first node of every stage $s_{j} \in \operatorname{stages}(b)$ such that $j \geq i$ there is a selected formula $\mathrm{E}_{s_{j}}$ which satisfies that $\sigma_{1} \mathcal{U}\left(\sigma_{2} \wedge \diamond \sigma_{3}\right)$ or some step-variant of it is in $\Pi_{s_{j}}$. Hence, except for a finite number of applications of $(\mathrm{EU} \sigma \square)^{+}$that extends the branch $b$ with some set $S_{\Sigma_{s_{j}}, \beta_{i}}^{+}$(where $\Sigma_{s_{j}}$ is the context of the selected formula at the first node of each stage $s_{j}$, in particular $\Sigma_{s_{i}}=\Sigma$ ) such that $1 \leq i \leq n$, the branch $b$ is repeatedly extended with some set $S_{\Sigma_{s_{j}}, \beta_{i}}^{+}$such that $n+1 \leq i \leq 2 n$, which includes a next-step variant of at least one formula in $\Pi_{s_{j}}$. Note that this next-step variant could be of $\sigma_{1} \mathcal{U}\left(\sigma_{2} \wedge \diamond \sigma_{3}\right)$ or some other formula of the form $\sigma \mathcal{U}(\sigma \wedge \diamond \sigma)$ or $\sigma \mathcal{U} \square \sigma$. In any case, the uniform set labelling the first node of each stage $s_{j}(i \leq j)$ has the form $\Sigma_{s_{j}}$, EП $\prod_{s_{j}}$ where $\Pi_{s_{j}}$ contains at least one next-step variant of $\sigma_{1} \mathcal{U}\left(\sigma_{2} \wedge \diamond \sigma_{3}\right), E \Pi_{s_{j}}$ is the selected formula and for every (except a finite number of) $\Sigma_{s_{k}}$ where $i \leq k \leq j, \Pi_{s_{j}}$ contains a formula of the form $\left.\sigma \wedge\left(\neg \Phi_{1} \wedge \cdots \wedge \neg \Phi_{r}\right)\right) \mathcal{U} \chi$ such that $\Phi_{h}=\Sigma_{s_{k}}$ for some $1 \leq h \leq r$. Since no other $\beta^{+}$-rule is applied each $\Sigma_{s_{j}}$ is a subset of the finite set formed by all state formulae that are subformulae of some formula in $\Sigma_{s_{i}} \cup \Pi$ and their negations. Hence, there exists a finite number of different $\Sigma_{s_{j}}$. Therefore, after finitely many applications of the $\beta^{+}$-rule $(\mathrm{E} \mathcal{U} \sigma \square)^{+}$, for some $k>=i, \Sigma_{s_{k}}=\Sigma_{s_{h}}$ for some $h \in\{i, . . k-1\}$, and $\neg \Sigma_{s_{k}} \in \tau\left(s_{k}\right)$, hence, $\Sigma_{s_{k}}$ must be inconsistent. Since $b$ is open, this is a contradiction. It means that for some $k \geq i$, the application of the corresponding $\beta^{+}$-rule $(\mathrm{E} \mathcal{U} \sigma \square)^{+}$should produce a node whose label contains $S_{\Sigma_{s_{j}}, \beta_{i}}^{+}$where $1 \leq i \leq n$. Henceforth, the open branch $b$ must satisfy (a).

If $E \Pi$ has the lowest priority, then $\Pi$ contains at least one $\square(\sigma \mathcal{U} \sigma)$ (and none $\sigma \mathcal{U}(\sigma \wedge \diamond \sigma)$ and none $\sigma \mathcal{U} \square \sigma$ ). Let us suppose there are $n \geq 1$ formulae, i.e. $\mathrm{E} \Pi=\mathrm{E}\left(\square\left(\sigma_{1}^{1} \mathcal{U} \sigma_{2}^{1}\right), \ldots, \square\left(\sigma_{1}^{n} \mathcal{U} \sigma_{2}^{n}\right), \Pi^{\prime}\right)$ for some $\Pi^{\prime}$ that does not contain any eventuality. Then, the $\alpha$-rule $(\mathrm{E} \square \mathcal{U})$ is applied $n$ times transforming the selected $\mathrm{E} \Pi$ into

$$
\mathrm{E}\left(\sigma_{1}^{1} \mathcal{U} \sigma_{2}^{1}, \ldots, \sigma_{1}^{n} \mathcal{U} \sigma_{2}^{n}, \mathrm{O} \square\left(\sigma_{1}^{1} \mathcal{U} \sigma_{2}^{1}\right), \ldots, \mathrm{O} \square\left(\sigma_{1}^{n} \mathcal{U} \sigma_{2}^{n}\right), \Pi^{\prime}\right)
$$

which has the highest priority. Hence, by a particular application of the case (a), for all $1 \leq j \leq n: \sigma_{2}^{j} \in \tau\left(s_{k}\right)$ for some stage $s_{k} \in \operatorname{stages}(b)(k \geq i)$. 
For A-disjunctive formulae, not only the outer context, but also the inner context, plays an important role. The next propositions explain the role of both kinds of contexts.

Proposition 45. Let b be an open branch of $\mathcal{A}_{\Sigma_{0}}^{\text {sys }}$, let $s_{i} \in \operatorname{stages}(b)$ and let $\Sigma_{s_{i}} \cup\{\mathrm{A \Pi}\}$ be the uniform set labelling the first node of $s_{i}$ where $\mathrm{A} \Pi$ is selected and some eventuality $\pi_{\mathcal{U}} \in \Pi$ is marked. Let be any branch where the next-steps variants of $\pi_{\mathcal{U}}$ are successively marked and $\Pi_{s_{i}}=\Pi \backslash\left\{\pi_{\mathcal{U}}\right\}$ is the initial inner context. Then, one of the following two facts hold:

(a) There exists $k \geq i$ and some $\Pi^{\prime} \in \operatorname{Variants}\left(\Pi \backslash\left\{\pi_{\mathcal{U}}\right\}\right)$ such that:

(al) $\left\{\sigma_{2}, \mathrm{~A}\left(\diamond \sigma_{3}, \Pi^{\prime}\right)\right\} \subseteq \tau\left(s_{k}\right)$ if $\pi_{\mathcal{U}}=\sigma_{1} \mathcal{U}\left(\sigma_{2} \wedge \diamond \sigma_{3}\right)$.

(a2) $\mathrm{A}\left(\square \sigma_{2}, \Pi^{\prime}\right) \in \tau\left(s_{k}\right)$ if $\pi_{\mathcal{U}}=\sigma_{1} \mathcal{U} \square \sigma_{2} \in \Pi^{\prime}$.

(a3) $\sigma_{2} \in \tau\left(s_{k}\right)$ if $\pi_{\mathcal{U}}=\square\left(\sigma_{1} \mathcal{U} \sigma_{2}\right) \in \Pi^{\prime}$.

(b) There exists $k \geq i$ such that the first node of $s_{k}$ is a loop-node whose companion node is in $s_{h}$, for some $h \in$ $\{i . . k-1\}$, some $\Pi^{\prime} \in \operatorname{Variants}\left(\Pi \backslash\left\{\pi_{\mathcal{U}}\right\}\right)$ and some next-step variant $\pi_{\mathcal{U}}^{v}$ of $\pi_{\mathcal{U}}$ such that $\mathrm{A}\left(\pi_{\mathcal{U}}^{v}, \Pi^{\prime}\right) \in \tau\left(s_{j}\right)$ for all $j \in\{h . . k\}$, and $\varphi_{\Pi^{\prime}} \in \tau\left(s_{k}\right)$

Moreover, in both cases, for all $j \in\{i . . k-1\}$ :

1. $\sigma_{1} \in \tau\left(s_{j}\right)$ for all $\sigma_{1} \mathcal{U}\left(\sigma_{2} \wedge \diamond \sigma_{3}\right) \in \Pi^{\prime} \cup\left\{\pi_{\mathcal{U}}\right\}$,

2. $\left\{\sigma_{1}, \sigma_{2}\right\} \subseteq \tau\left(s_{j}\right)$ for all $\sigma_{1} \mathcal{U} \square \sigma_{2} \in \Pi^{\prime} \cup\left\{\pi_{\mathcal{U}}\right\}$,

3. $\sigma_{1} \in \tau\left(s_{j}\right)$ or $\sigma_{2} \in \tau\left(s_{j}\right)$ for all $\square\left(\sigma_{1} \mathcal{U} \sigma_{2}\right) \in \Pi^{\prime} \cup\left\{\pi_{\mathcal{U}}\right\}$, and

4. $\sigma_{1} \in \tau\left(s_{j}\right)$ for all $\square\left(\sigma_{1} \vee \square \sigma_{2}\right) \in \Pi^{\prime}$.

PROOF. If $\varphi_{\pi_{s_{i}}}=\mathbf{F}$, the uniform set labelling the first node at each stage $s_{j}(j \geq i)$ of $b$ has the form

$$
\Sigma_{s_{j}}, \mathrm{~A}\left(\left(\sigma_{1} \wedge\left(\neg \Sigma_{s_{i}} \wedge \cdots \wedge \neg \Sigma_{s_{j-1}}\right)\right) \mathcal{U} \chi, \Pi_{s_{j}}\right)
$$

where each $\Sigma_{s_{j}}$ is the outer (resp. inner) context of the selected formula containing the marked next-step variant of $\pi_{\mathcal{U}}$ ) at the first node of each stage $s_{j}$. In particular, $\Sigma_{s_{i}}=\Sigma$. Since no other $\beta^{+}$-rule is applied each $\Sigma_{s_{j}}$ is a subset of the finite set formed by all state formulae that are subformulae of some formula in $\Sigma_{s_{i}} \cup \Pi$ and their negations. Hence, there are a finite number of different $\Sigma_{s_{j}}$. Therefore, after finitely many applications of the $\beta^{+}$-rule, $\Sigma_{s_{h}}=\Sigma_{s_{j}}$, for some $h>=i$, for some $j \in\{i, . . h-1\}$, and $\sigma_{1} \wedge\left(\neg \Sigma_{s_{i}} \wedge \cdots \wedge \neg \Sigma_{s_{h-1}}\right) \in \tau\left(s_{h}\right)$. In particular, $\neg \Sigma_{s_{h}} \in \tau\left(s_{h}\right)$, hence, $\Sigma_{s_{h}}$ must be inconsistent. Since $b$ is open, this is a contradiction. This means that, for some $k \geq i$ the application of the corresponding $\beta^{+}$-rule should produce a node generated by the corresponding set $S_{\beta_{1}}^{+}$. Henceforth, the open branch $b$ must satisfy (a1) or (a2) or (a3), depending on the case of $\pi_{\mathcal{U}}$.

If $\varphi_{\pi_{s_{i}}} \neq \mathbf{F}$, then, the uniform set labelling the first node at each stage $s_{j}(j \geq i)$ has the form

$$
\Sigma_{s_{j}}, \mathrm{~A}\left(\left(\sigma_{1} \wedge\left(\neg \Sigma_{s_{i}} \vee \varphi_{\Pi_{s_{i}}}\right) \wedge \cdots \wedge\left(\neg \Sigma_{s_{j-1}} \vee \varphi_{\Pi_{s_{j-1}}}\right)\right) \mathcal{U} \chi, \Pi_{s_{j}}\right)
$$

where each $\Pi_{s_{h}}$ is the inner context at the first node of each stage $s_{h}$. In particular, $\Pi_{s_{i}}=\Pi \backslash\left\{\pi_{\mathcal{U}}\right\}$. Since no other $\beta^{+}$-rule is applied, then

- each $\Sigma_{s_{j}}$ is a subset of the following finite set $L C(\Sigma, \Pi): L C(\Sigma, \Pi)$ is formed by all state formulae that are subformulae of some formula in $\Sigma \cup \Pi$, their negations, and a formula $\mathrm{E} \diamond \sigma_{2}$ for each subformula $\square\left(\sigma_{1} \mathcal{U} \sigma_{2}\right)$ in $\Pi$ (see Definition 19), and

- each $\Pi_{s_{j}}$ is a subset of the finite set of all state formulae that are subformulae of some formula in $\Pi$. Indeed, each $\Pi_{s_{j+1}} \in \operatorname{Variants}\left(\Pi_{s_{j}}\right)$ for all $j \geq i$.

In particular, there are a finite number of different outer and inner contexts. Henceforth, there are two possibilities. First, for some $h, k$ such that $k>h \geq i$, both $\Sigma_{s_{k}}=\Sigma_{s_{h}}$ and $\Pi_{s_{k}}=\Pi_{s_{h}}$; and second for some $h \geq i$, the formula $\varphi_{\pi_{s_{h}}}$ is $\mathbf{F}$. In the latter case, the item (a) must be satisfied for some $k \geq h$. In the former case, by Definition 41 and Proposition 43 for all $j \in\{h . . k\}: \Pi_{s_{j}}=\Pi_{s_{k}}$. Let $\Pi^{\prime}=\Pi_{s_{k}}$, then the first nodes at the sequence of stages $s_{h}, s_{h+1}, \ldots, s_{k}$ are respectively labelled by

$$
\Sigma_{s_{h}} \cup\left\{\mathrm{A}\left(\left(\sigma_{1} \wedge \delta\right) \mathcal{U} \chi, \Pi^{\prime}\right)\right\}, \Sigma_{s_{h+1}} \cup\left\{\mathrm{A}\left(\left(\sigma_{1} \wedge \delta\right) \mathcal{U} \chi, \Pi^{\prime}\right)\right\}, \ldots, \Sigma_{s_{k}} \cup\left\{\mathrm{A}\left(\left(\sigma_{1} \wedge \delta\right) \mathcal{U} \chi, \Pi^{\prime}\right)\right\}
$$

where $\delta=\left(\neg \Sigma_{s_{i}} \vee \varphi_{\Pi_{s_{i}}}\right) \wedge \cdots \wedge\left(\neg \Sigma_{s_{h}} \vee \varphi_{\Pi^{\prime}}\right) \wedge \cdots \wedge\left(\neg \Sigma_{s_{k-1}} \vee \varphi_{\Pi^{\prime}}\right)$ or equivalently $\delta=\left(\neg \Sigma_{s_{i}} \vee \varphi_{\Pi_{s_{i}}}\right) \wedge \cdots \wedge$ $\left(\left(\neg \Sigma_{s_{h}} \wedge \cdots \wedge \neg \Sigma_{s_{z-1}}\right) \vee \varphi_{\Pi^{\prime}}\right)$. Hence, in node $s_{k}$, the application of the $\beta^{+}$-rule to the marked eventuality produces 640 a right-hand child that contains $\Sigma_{s_{k}}$ and $\sigma_{1} \wedge \delta$. Therefore, by rules $(\wedge)$ and $(\vee)$, it also contains $\neg \Sigma_{s_{h}}=\neg \Sigma_{s_{k}}$. 
Therefore, since $b$ is open, $\tau\left(s_{k}\right)$ must contain $\varphi_{\Pi^{\prime}}$, which completes the proof of item (b). Moreover, in both cases (a) and (b), for all $j \in\{i . . k-1\}$, each inner context $\Pi_{s_{j}+1}$ satisfies the properties of $\Pi^{\prime}$ in Proposition 42 with respect to $\Pi_{s_{j}}$ as $\Pi$. Consequently, the last four items of the proposition hold.

The next two propositions provide a detailed description of how the highest priority formulae evolve in open branches.

Proposition 46. Let $b$ be an open branch of $\mathcal{A}_{\Sigma_{0}}^{\text {sys }}$, and let $\mathrm{E} \Pi$ be of the highest priority that is selected at some stage $s_{i} \in \operatorname{stages}(b)$. Then there exists a state $s_{k} \in \operatorname{stages}(b)$ (for some $k \geq i$ ) and some (possibly empty and minimal) set $\Pi^{\prime} \in \operatorname{Variants}(\Pi)$ such that $\mathrm{E} \Pi^{\prime} \in \tau\left(s_{k}\right), \mathrm{E} \Pi^{\prime}$ is of the lowest priority and for all $\pi \in \Pi$ the following facts hold:

(a) If $\pi=\sigma_{1} \mathcal{U}\left(\sigma_{2} \wedge \diamond \sigma_{3}\right)$ then there exists $j, j^{\prime}$ such that $i \leq j \leq j^{\prime} \leq k$, for all $h \in\{i, \ldots, j-1\}: \sigma_{1} \in \tau\left(s_{h}\right)$, $\sigma_{2} \in \tau\left(s_{j}\right)$, and $\sigma_{3} \in \tau\left(s_{j^{\prime}}\right)$.

(b) If $\pi=\sigma_{1} \mathcal{U} \square \sigma_{2}$ then there exists $j$ such that $i \leq j \leq k$ and for all $h \in\{i, \ldots, j-1\}: \sigma_{1} \in \tau\left(s_{h}\right)$, and for all $h \in\{j, \ldots, k\}: \sigma_{2} \in \tau\left(s_{h}\right)$ and $\square \sigma_{2} \in \Pi^{\prime}$.

(c) if $\pi=\square\left(\sigma_{1} \mathcal{U} \sigma_{2}\right)$ then $\square\left(\sigma_{1} \mathcal{U} \sigma_{2}\right) \in \Pi^{\prime}$, and for all $j \in\{i, \ldots, k\}$ : either $\sigma_{1} \in \tau\left(s_{j}\right)$ or $\sigma_{2} \in \tau\left(s_{j}\right)$.

(d) if $\pi=\square\left(\sigma_{1} \vee \square \sigma_{2}\right)$ then one of the following two facts holds:

$655 \quad$ (dl) For all $j \in\{i, \ldots, k\}: \sigma_{1} \in \tau\left(s_{j}\right)$ and $\square\left(\sigma_{1} \vee \square \sigma_{2}\right) \in \Pi^{\prime}$.

(d2) There exists $j$ such that $i \leq j \leq k$ and for all $h \in\{i, \ldots, j-1\}: \sigma_{1} \in \tau\left(s_{h}\right)$, and for all $h \in\{j, \ldots, k\}$ : $\sigma_{2} \in \tau\left(s_{h}\right)$ and $\square \sigma_{2} \in \Pi^{\prime}$.

PROOF. By simultaneous induction on the structures of formulae in $\Pi$ and Propositions 44 and 42 (a) and (b)), the above items (a) and (b) hold for some $k \geq i$. Note that in case (a), $\mathrm{E}\left(\diamond \sigma_{3}, \Pi^{\prime}\right)$ (for some $\Pi^{\prime}$ such that $\left\{\diamond \sigma_{3}\right\} \cup \Pi^{\prime} \in$ Variants $(\Pi)$ ) is kept selected at stage $s_{j}$. Hence, the eventuality $\sigma_{3} \in \tau\left(s_{j^{\prime}}\right)$ for some $j^{\prime} \geq j$. Therefore, by Proposition 44, the existence of such $j^{\prime}$ is ensured. In case (b) $\mathrm{E}\left(\square \sigma_{2}, \Pi^{\prime}\right) \in \tau\left(s_{j}\right)$ for some $j \geq i$, hence, by Proposition 42 d), for all $h \in\{j, \ldots, k\}: \sigma_{2} \in \tau\left(s_{h}\right)$ and $\square \sigma_{2} \in \Pi^{\prime}$. Items (c) and (d) are ensured by Propositions 42 (c) and (d). Additionally, by minimality, $\Pi^{\prime}$ only contains formulae of the forms $\square\left(\sigma_{1} \mathcal{U} \sigma_{2}\right)$ and $\square\left(\sigma_{1} \vee \square \sigma_{2}\right)$, hence $\mathrm{E} \Pi^{\prime}$ is of the priority.

The other kind of the highest-priority formulae are $A \Pi$ such that $\Pi$ is exclusively formed by formulae of the form $\sigma_{1} \mathcal{U}\left(\sigma_{2} \wedge \diamond \sigma_{3}\right)$

Proposition 47. Let $b$ be an open branch of $\mathcal{A}_{\Sigma_{0}}^{\text {sys }}$, and let $\mathrm{A} \Pi$ be a formula of the highest priority that is selected at some stage $s_{i} \in \operatorname{stages}(b)$. Then there exists $\pi=\sigma_{1} \mathcal{U}\left(\sigma_{2} \wedge \nabla_{3}\right) \in \Pi$ and some stage $s_{k} \in \operatorname{stages}(b)$ (for some $k \geq i)$ such that for all $j \in\{i, \ldots, k-1\}: \sigma_{1} \in \tau\left(s_{j}\right),\left\{\sigma_{2}, \mathrm{~A}\left(\diamond \sigma_{3}, \Pi^{\prime}\right)\right\} \subseteq \tau\left(s_{k}\right)$ for some $\Pi^{\prime} \in \operatorname{Variants}(\Pi \backslash\{\pi\})$. Moreover, $\mathrm{A}\left(\diamond \sigma_{3}, \Pi^{\prime}\right)$ is also a formula of the highest priority.

Proof. According to Definitions 24 and 26, one eventuality in $\Pi$ must be marked at the stage $s_{i}$ of $b$. Hence, there exists $\sigma_{1} \mathcal{U}\left(\sigma_{2} \wedge \diamond \sigma_{3}\right) \in \Pi$ that is the marked eventuality at stage $s_{i}$. Since $\varphi_{\Pi}$ is $\mathbf{F}$ when $\Pi$ is exclusively formed by formulae of the form $\sigma_{1} \mathcal{U}\left(\sigma_{2} \wedge \diamond \sigma_{3}\right)$, the item (a) of Proposition 45 holds. Hence, by Proposition 45 (a1), there exists $k \geq i$ and $\Pi^{\prime}$ such that $\left\{\sigma_{2}, \mathrm{~A}\left(\diamond \sigma_{3}, \Pi^{\prime}\right)\right\} \in \tau\left(s_{k}\right)$. Since $\Pi^{\prime} \in \operatorname{Variants}(\Pi \backslash\{\pi\})$, every formula in $\Pi^{\prime}$ is 675 of the form $\sigma_{1} \mathcal{U}\left(\sigma_{2} \wedge \diamond \sigma_{3}\right)$ (in particular, $\diamond \sigma$ which abbreviates $\left.\mathbf{T} \mathcal{U}(\sigma \wedge \diamond \mathbf{T})\right)$. Hence, $\mathrm{A}\left(\diamond \sigma_{3}, \Pi^{\prime}\right)$ is also of the highest-priority.

Next, we show that any open branch of $\mathcal{A}_{\Sigma_{0}}^{\text {sys }}$ is eventuality-covered. In the sequel, we deal with uniform sets formed by non-selectable and the lowest priority formulae (i.e, without the highest-priority formulae), we call them cycle-uniform sets.

Proposition 48. Any open branch b of $\mathcal{A}_{\Sigma_{0}}^{\text {sys }}$ is eventuality-covered.

PROOF. Let $b$ be any open branch of $\mathcal{A}_{\Sigma_{0}}^{\text {sys }}$, we are going to show that there must exist some stage $s_{\ell}$ in $b$ with the first node $n_{\ell}$ labelled by a cycle-uniform set $\Sigma_{\ell}$ such that any selection of a formula of the lowest priority in $\Sigma_{\ell}$ produces a loop-node whose companion node is $n_{\ell}$.

Let $\Sigma$ and $\Sigma^{\prime}$ be any two cycle-uniform sets of state formulae, we say that $\Sigma^{\prime} \preceq \Sigma$ iff every formula in $\Sigma^{\prime}$ is either a proper subformula of some formula $\mathrm{Q} \Pi \in \Sigma$ or its negation, or a formula $\mathrm{Q} \Pi^{\prime}$ such that there exists $\mathrm{Q} \Pi \in \Sigma$ such that $\Pi^{\prime} \in \operatorname{Variants}(\Pi)$. It is worth noting that the formulae $\mathrm{E} \vee \sigma_{2}$ that can be introduced by $\varphi_{\Pi}$ (see Definition 19 ) are of the highest priority, then they cannot belong to any cycle-uniform set. Let $b=s_{0}, \ldots, s_{i}, \ldots, s_{j}, \ldots(0 \geq i>j)$ be any open branch of $\mathcal{A}_{\Sigma_{0}}^{\text {sys }}$, and let $\Sigma$ and $\Sigma^{\prime}$ respectively be the cycle-uniform sets labelling the first node of $s_{i}$ and 
$s_{j}$. By Propositions 42, 43, 44 and 45, $\Sigma^{\prime} \preceq \Sigma$. Moreover, for any cycle-uniform set $\Sigma, \preceq$ is a well-founded order on 690 the collection of all cycle-uniform sets $\Sigma^{\prime}$ such that $\Sigma^{\prime} \preceq \Sigma$.

Let $b=s_{0}, \ldots, s_{i}, \ldots, s_{j}, \ldots, s_{k}, \ldots(0 \geq i>j \geq k)$ be any open branch of $\mathcal{A}_{\Sigma_{0}}^{\text {sys }}$. Suppose that every highest priority formula in the initial uniform set has been selected before the stage $s_{i}$. Let $\Sigma$ be the cycle-uniform set labelling the first node of $s_{j}$ which is a loop-node whose companion is the first node of $s_{i}$. Suppose that $\Sigma$ contains at least one lowest priority formula $\mathrm{A} \Pi$ that was selected at $s_{i}$. If $b$ is not already eventuality-covered, this means that there exists $\mathrm{A} \Pi^{\prime} \in \Sigma$ of the lowest priority that has not been selected. Suppose that $A \Pi^{\prime}$ is selected at $s_{j}$ and there exists a loop-node at $s_{k}$ labelled by $\Sigma^{\prime}$ then, $\Sigma^{\prime} \preceq \Sigma$. If $\Sigma=\Sigma^{\prime}$ and there are no more selectable formula in $\Sigma, b$ is already eventuality covered and the first node of $s_{i}$ is $n_{\ell}$. Otherwise, $\Sigma^{\prime} \prec \Sigma$, so that the companion node of the first node of $s_{k}$ is the first node of some stage $s_{h}$ such that $h>i$. In general, for any number of the lowest priority formulae in $\Sigma$, by well-foundness of $\prec$ there should exist a minimal node $n_{\ell}$ labelled by a cycle-uniform set $\Sigma_{\ell}$ such 700 that any selection of the lowest priority formula in $\Sigma_{\ell}$ produces a loop-node whose companion node is $n_{\ell}$. Hence, the branch $b$ ends by a subsequence of $n \geq 2$ (possibly non-consecutive) stages $s_{i_{1}}, \ldots, s_{i_{n}}$ whose first node is labelled by $\Sigma_{\ell}$, where $n_{\ell}$ is the first node of $s_{i_{1}}$, and each selectable (lowest priority) formula in $\Sigma_{\ell}$ is selected at some stage in $s_{i_{1}}, \ldots, s_{i_{n}}$. In particular, $\Sigma_{\ell}$ could be empty, then $n=2$ and $b$ is trivially eventuality-covered.

\subsection{Refutational Completeness}

705 In this subsection we prove that our tableau method is refutationally complete, that is if a set of state formulae is unsatisfiable then there exists a closed tableau for it. For that, we first ensure the existence of a model for any open bunch of $\mathcal{A}_{\Sigma_{0}}^{\text {sys }}$.

Lemma 49 (Model Existence). Let $H$ be an expanded bunch of $\mathcal{A}_{\Sigma_{0}}^{\text {sys }}$ and $\mathcal{K}_{H}=(S, R, L)$ be as in Definition 39 . For every state $s \in S$, if $\sigma \in L(s)$ then $\mathcal{K}_{H}, s, 0 \models \sigma$. Therefore, $\mathcal{K}_{H} \mid=\Sigma$.

710 PROOF. Let $H$ be any expanded bunch of $\mathcal{A}_{\Sigma_{0}}^{\text {sys }}$ and let $b$ be any open branch in $H$. The construction of any branch of $\mathcal{A}_{\Sigma_{0}}^{\text {sys }}$ starts by selecting a formula of the highest-priority (if any) and marking eventualities as explained in Definition 24. At most one eventuality is marked inside the unique selected QП and the rules of Figure 7 are applied to this formula and to no one else. When a $\beta^{+}$-rule is applied to a formula of the highest priority (independently of the marked eventuality), then only the outer context (but no the inner context) is used to construct the new-step variant. 715 Therefore, while some highest priority formulae is selected, previous labels cannot be repeated. Consequently, the initial segment of any open branch has no loop-nodes. This initial segment can be empty or not. According to Proposition 48, the branch $b$ is eventuality covered. Hence, there exists a (possibly empty) cycle-uniform set $\Sigma_{\ell}$ such that for some $i \geq 0: b=s_{0}, s_{1}, \ldots, s_{i-1}, s_{i}, s_{i+1}, \ldots, s_{j}, n_{\ell}$, where each $s_{h}$ stands for a stage and $n_{\ell}$ is a nonexpandable loop-node labelled by $\Sigma_{\ell}$ whose companion node is the first node at stage $s_{i}$. Let $s_{i_{1}}, s_{i_{2}}, \ldots, s_{i_{z}}$ be a the 720 subsequence formed by all the stages in $s_{i}, s_{i+1}, \ldots, s_{j}$ whose first node is labelled by $\Sigma_{\ell}$ (in particular, $s_{i_{1}}=s_{i}$ ). Then, each lowest priority formula in $\Sigma_{\ell}$ has been selected at some node $n_{h}(h \in i . . j+1)$. The tableau branch $b$ represents a cyclic branch (of a model) such that path $(b)=s_{0}, s_{1}, \ldots, s_{i-1}\left\langle s_{i}, s_{i+1}, \ldots, s_{j}\right\rangle^{\omega}$ where each state $s_{h}$ ( $h \in 0 . . j$ ) is labelled by the set of all atoms occurring in the label $\tau\left(s_{h}\right)$ of the tableau stage $s_{h}$ (in the label of some tableau node at stage $s_{h}$ ).

725 We are going to prove that $\mathcal{K}_{H}, s_{a}, 0 \models \sigma$ for any $a \in 0 \ldots j$ and any formula $\sigma$ in $\tau\left(s_{a}\right)$, by structural induction on the formula $\sigma$. The base of the induction $\sigma=p \in$ Prop is ensured by Definition $39, \mathcal{K}_{H}, s_{a}, 0=p$.

The bunch $H$ allows us to ensure that whenever a tableau node in stage $s_{a}$ is labelled by an elementary set $\left\{\Sigma, \mathrm{A} \circ \Phi_{1}, \ldots, \mathrm{A} \circ \Phi_{n}, \mathrm{E} \circ \Psi_{1}, \ldots, \mathrm{E} \circ \Psi_{m}\right\} \subseteq L(s)$ then, by rule $(\mathrm{Q} \circ)$, the bunch $H$ contains one successor stage $s_{a+1}^{i}$, for each $i \in 1 . . m$, that contains $\left\{A \Phi_{1}, \ldots, \mathrm{A} \Phi_{n}, \mathrm{E} \Psi_{i}\right\}$. Since by induction hypothesis, we can assume that ${ }_{730} \mathcal{K}_{H}, s_{a+1}^{i}, 0 \models A \Phi_{1}, \ldots, \mathrm{A} \Phi_{n}, \mathrm{E} \Psi_{i}$ for all $i \in 1 . . m$, and $\Sigma$ is a consistent set of literals, then we can infer that $\mathcal{K}_{H}, s_{a}, 0 \models\left\{\Sigma, \mathrm{A} \circ \Phi_{1}, \ldots, \mathrm{A} \circ \Phi_{n}, \operatorname{E\circ } \Psi_{1}, \ldots, \operatorname{E\circ } \Psi_{m}\right\}$.

To complete the proof, we prove different cases for $\sigma$ being a formula of the form QП, depending on whether $\sigma$ is selectable or not and, in the selectable case, depending on the $\sigma$ priority for the selection strategy.

Let $\sigma=\mathrm{E} \Pi \in \tau\left(s_{a}\right)$ be non-selectable. Then every $\pi \in \Pi$ is of the form $\square\left(\sigma_{1} \vee \square \sigma_{2}\right)$. Hence, by Proposition 42 735 (d) and the induction hypothesis, there exists a state $s_{k}$ (for some $k \geq a$ ) and some non-empty set $\Pi^{\prime} \in V$ ariants $(\Pi$ ) such that $\mathcal{K}_{H}, s_{k}, 0 \models \mathrm{E} \Pi^{\prime}, \square\left(\sigma_{1} \vee \square \sigma_{2}\right) \in \Pi^{\prime}$, and for all $\pi \in \Pi$ :

- $\mathcal{K}_{H}, s_{j}, 0 \models \sigma_{1}$ for all $a \leq j \leq k$, and 
- there exists $j$ such that $a \leq j \leq k$ and for all $h \in a . . j-1: \mathcal{K}_{H}, s_{h}, 0=\sigma_{1}$, and for all $h \in j . . k: \mathcal{K}_{H}, s_{h}, 0 \models \sigma_{2}$ and $\square \sigma_{2} \in \Pi^{\prime}$.

Therefore, $\mathcal{K}_{H}, s_{a}, 0 \models \mathrm{E} \Pi$.

Let $\sigma=\mathrm{A} \Pi \in \tau\left(s_{a}\right)$ be non-selectable. Then every $\pi \in \Pi$ is of the form $\square\left(\sigma_{1} \vee \square \sigma_{2}\right)$. By Proposition 43 (d) and the induction hypothesis, there exists a state $s_{k}$ (for some $k \geq a$ ) and some non-empty set $\Pi^{\prime} \in \operatorname{Variants}(\Pi)$ such that $\mathcal{K}_{H}, s_{k}, 0=\mathrm{A} \Pi^{\prime}$ and for all $\pi=\square\left(\sigma_{1} \vee \square \sigma_{2}\right) \in \Pi$ :

- If $\square\left(\sigma_{1} \vee \square \sigma_{2}\right) \in \Pi^{\prime}$ then $\mathcal{K}_{H}, s_{j}, 0 \models \sigma_{1}$ for all $j \in a . . k$, and

- if $\square \sigma_{2} \in \Pi^{\prime}$ then there exists $j$ such that $a \leq j \leq k$ and for all $h \in a . . j-1: \mathcal{K}_{H}, s_{h}, 0 \models \sigma_{1}$, and for all $h \in j . . k: \mathcal{K}_{H}, s_{h}, 0 \models \sigma_{2}$.

Therefore, $\mathcal{K}_{H}, s_{a}, 0 \mid \mathrm{A} \Pi$.

Let $\sigma=\mathrm{E} \Pi \in \tau\left(s_{a}\right)$ be a (selectable) formula of the highest priority. According to Proposition 46 and the induction hypothesis, there exists a state $s_{k}$ (for some $k \geq a$ ) and some (possibly empty and minimal) set $\Pi^{\prime} \in$ Variants $(\Pi)$ such that $\mathcal{K}_{H}, s_{k}, 0 \models \mathrm{E} \Pi^{\prime}$ and for all $\pi \in \Pi$ the following facts hold:

- If $\pi=\sigma_{1} \mathcal{U}\left(\sigma_{2} \wedge \diamond \sigma_{3}\right)$ then there exists $j, j^{\prime}$ such that $a \leq j \leq j^{\prime} \leq k$, for all $h \in i . . j-1: \mathcal{K}_{H}, s_{h}, 0 \mid=\sigma_{1}$, $\mathcal{K}_{H}, s_{j}, 0 \models \sigma_{2}$, and $\mathcal{K}_{H}, s_{j^{\prime}}, 0 \models \sigma_{3}$.

- If $\pi=\sigma_{1} \mathcal{U} \square \sigma_{2}$ then there exists $j$ such that $a \leq j \leq k$ and for all $h \in a . . j-1: \mathcal{K}_{H}, s_{h}, 0 \models \sigma_{1}$, and for all $h \in j . . k: \mathcal{K}_{H}, s_{h}, 0 \models \sigma_{2}$ and $\square \sigma_{2} \in \Pi^{\prime}$.

- If $\pi=\square\left(\sigma_{1} \mathcal{U} \sigma_{2}\right)$ then $\square\left(\sigma_{1} \mathcal{U} \sigma_{2}\right) \in \Pi^{\prime}$, and for all $j \in a . . k$ : either $\mathcal{K}_{H}, s_{j}, 0 \models \sigma_{1}$ or $\mathcal{K}_{H}, s_{a}, 0 \models \sigma_{2}$.

- If $\pi=\square\left(\sigma_{1} \vee \square \sigma_{2}\right)$ then one of the following two facts holds:

- For all $j \in a . . k: \mathcal{K}_{H}, s_{j}, 0 \models \sigma_{1}$ and $\square\left(\sigma_{1} \vee \square \sigma_{2}\right) \in \Pi^{\prime}$.

- There exists $j$ such that $a \leq j \leq k$ and for all $h \in a . . j-1: \mathcal{K}_{H}, s_{h}, 0 \models \sigma_{1}$, and for all $h \in j$.., $k$ : $\mathcal{K}_{H}, s_{h}, 0=\sigma_{2}$ and $\square \sigma_{2} \in \Pi^{\prime}$.

760 Therefore, $\mathcal{K}_{H}, s_{a}, 0 \models \mathrm{E} \Pi$.

Let $\sigma=\mathrm{A} \Pi \in \tau\left(s_{a}\right)$ be a formula of the highest-priority where $\sigma_{1} \mathcal{U}\left(\sigma_{2} \wedge \nabla_{3}\right) \in \Pi$ is marked. By Proposition 47 and the induction hypothesis, we have that $\mathcal{K}_{H}, s_{v}, 0 \models \sigma_{2}$ for some $v \geq a, \mathcal{K}_{H}, s_{z}, 0 \models \sigma_{1}$ for all $z \in a . . v-$ 1. In addition, $\mathrm{A}\left(\diamond \sigma_{3}, \Pi^{\prime}\right) \in \tau\left(s_{v}\right)$ (which is also the highest priority formula). Hence, by induction hypothesis, $\mathcal{K}_{H}, s_{v}, 0=\mathrm{A}\left(\diamond \sigma_{3}, \Pi^{\prime}\right)$. Additionally, by Proposition 43 and the induction hypothesis:

(a) For all $\sigma_{1}^{\prime} \mathcal{U}\left(\sigma_{2}^{\prime} \wedge \diamond \sigma_{3}^{\prime}\right) \in \Pi$ :

- If $\sigma_{1}^{\prime} \mathcal{U}\left(\sigma_{2}^{\prime} \wedge \diamond \sigma_{3}^{\prime}\right)$ is also in $\Pi^{\prime}$, then $\mathcal{K}_{H}, s_{z}, 0 \models \sigma_{1}^{\prime}$ for all $z \in a . . v-1$.

- If $\diamond \sigma_{3}^{\prime} \in \Pi^{\prime}$ then $\mathcal{K}_{H}, s_{z}, 0 \models \sigma_{2}^{\prime}$ for some $z \in a$..v-1.

(b) For all $\sigma_{1}^{\prime} \mathcal{U} \square \sigma_{2}^{\prime} \in \Pi$ :

- If $\sigma_{1}^{\prime} \mathcal{U} \square \sigma_{2}^{\prime}$ ' is also in $\Pi^{\prime}$, then $\mathcal{K}_{H}, s_{z}, 0=\sigma_{1}^{\prime}$ for all $z \in a . . v-1$

- if $\square \sigma_{2}^{\prime} \in \Pi^{\prime}$ then there exists $j \in a . . v-1$ such that $\mathcal{K}_{H}, s_{j}, 0 \models \sigma_{1}^{\prime}$ for all $z \in a . . j-1$ and $\mathcal{K}_{H}, s_{z}, 0 \models \sigma_{2}^{\prime}$ for all $z \in j . . v$.

Therefore, $\mathcal{K}_{H}, s_{a}, 0 \models \mathrm{A} \Pi$.

Let $\sigma=\mathrm{E} \Pi \in \tau\left(s_{a}\right)$ be a (selectable) formula of the lowest priority. If $a<i$ then, by Proposition 42 ((c) and (d)), there exists non-empty $\Pi^{\prime} \in$ Variants $(\Pi)$ such that $\mathrm{E} \Pi^{\prime} \in \tau\left(n_{i}\right) \subseteq \tau\left(s_{i}\right)$ and

- For all $\square\left(\sigma_{1} \mathcal{U} \sigma_{2}\right) \in \Pi: \square\left(\sigma_{1} \mathcal{U} \sigma_{2}\right) \in \Pi^{\prime}$ and for all $z \in a . . i-1: \sigma_{1} \in \tau\left(s_{z}\right)$ or $\sigma_{2} \in \tau\left(s_{z}\right)$.

- For all $\square\left(\sigma_{1} \vee \square \sigma_{2}\right) \in \Pi$ : either $\square\left(\sigma_{1} \vee \square \sigma_{2}\right) \in \Pi^{\prime}$ and $\sigma_{1} \in \tau\left(s_{z}\right)$ for all $z \in a . . i-1$; or $\square \sigma_{2} \in \Pi^{\prime}$ and there exists $j$ such that $a \leq j \leq i$, so that for all $h \in a . . j-1: \sigma_{1} \in \tau\left(s_{h}\right)$ and for all $h \in j . . i: \sigma_{2} \in \tau\left(s_{h}\right)$.

Since $\mathrm{E} \Pi^{\prime} \in \tau\left(n_{1}\right)$ is selected at some node $n_{h}$ for some $i \leq h \leq j$, then by Proposition 44(c), $\sigma_{2} \in \tau\left(s_{w}\right)$ for some $w \in i . . j$. Moreover, by Proposition 42(c), for all $z \in i . . j: \sigma_{1} \in \tau\left(s_{z}\right)$ or $\sigma_{2} \in \tau\left(s_{z}\right)$. In addition $\tau\left(n_{h}\right)=\Sigma_{\ell}$ for all ${ }_{780} h \in i . . j+1$, henceforth by Proposition $42(\mathrm{~d})$, for all $\square\left(\sigma_{1} \vee \square \sigma_{2}\right) \in \Pi^{\prime}: \sigma_{1} \in \tau\left(s_{z}\right)$ for all $z \in i . . j$. Therefore, the application of the induction hypothesis to every $\sigma_{1}$ and every $\sigma_{2}$, allows us to ensure that $\mathcal{K}_{H}, s_{a}, 0 \models$ EП. The case $a \geq i$ can be seen as the particular case where $\Pi^{\prime}=\Pi$ and $\mathrm{E} \Pi \in \tau\left(n_{h}\right)$ for all $h \in i . . j+1$.

Let $\sigma=\mathrm{A} \Pi \in \tau\left(s_{a}\right)$ be a (selectable) formula of the lowest priority. Then, $\Pi$ contains at least one $\sigma_{1} \mathcal{U} \square \sigma_{2}$ or $\square\left(\sigma_{1} \mathcal{U} \sigma_{2}\right)$. We study two cases depending on whether there exists $\Pi^{\prime} \in \operatorname{Variants}(\Pi)$ such that $A \Pi^{\prime} \in \Sigma_{\ell}$ or not. In the negative case, by Proposition 43 and the induction hypothesis, there exists $\sigma_{1} \mathcal{U}\left(\sigma_{2} \wedge \diamond \sigma_{3}\right) \in \Pi$ and some $j, j^{\prime}$ such that $a \leq j \leq j^{\prime} \leq k$, for all $h \in i . . j-1: \mathcal{K}_{H}, s_{h}, 0 \models \sigma_{1}, \mathcal{K}_{H}, s_{j}, 0 \models \sigma_{2}$, and $\mathcal{K}_{H}, s_{j^{\prime}}, 0 \models$ $\sigma_{3}$. Therefore, $\mathcal{K}_{H}, s_{a}, 0 \models \mathrm{A} \Pi$. Otherwise, let $\mathrm{A}^{\prime} \in \Sigma_{\ell} \subseteq \tau\left(s_{i}\right)$ such that $\Pi^{\prime} \in \operatorname{Variants}(\Pi)$. By induction hypothesis and Proposition 43. $\mathcal{K}_{H}, s_{i}, 0 \models \mathrm{A} \Pi^{\prime}$ suffices to ensure that $\mathcal{K}_{H}, s_{a}, 0 \models \mathrm{A} \Pi$. Hence, we are going to prove that $\mathcal{K}_{H}, s_{i}, 0 \models \mathrm{A} \Pi^{\prime}$. If $\mathrm{A}^{\prime}$ is non-selectable, then $\mathcal{K}_{H}, s_{i}, 0 \models \mathrm{A} \Pi^{\prime}$ holds because $\mathcal{K}_{H}, s_{h}, 0 \models \sigma_{1}$ for all 

$s_{i}, s_{i+1}, \ldots, s_{j}$, hence for all $h \in i . . j$ :

- $\sigma_{1} \in \tau\left(s_{h}\right)$ for all $\sigma_{1} \mathcal{U}\left(\sigma_{2} \wedge \diamond \sigma_{3}\right) \in \Pi^{\prime}$. - $\left\{\sigma_{1}, \sigma_{2}\right\} \subseteq \tau\left(s_{h}\right)$ for all $\sigma_{1} \mathcal{U} \square \sigma_{2} \in \Pi^{\prime}$.

Moreover, $\mathrm{A} \Pi^{\prime}$ contains at least one $\sigma_{1} \mathcal{U} \square \sigma_{2}$ or $\square\left(\sigma_{1} \mathcal{U} \sigma_{2}\right)$, and one of its eventualities (that could be also of the form $\left.\sigma_{1} \mathcal{U}\left(\sigma_{2} \wedge \diamond \sigma_{3}\right)\right)$ is selected at some of the stages $s_{i_{1}}, s_{i_{2}}, \ldots, s_{i_{z}}$. Hence, Proposition 45, along with the induction hypothesis, ensure that $\mathcal{K}_{H}, s_{i}, 0 \models \mathrm{A} \Pi^{\prime}$.

Corollary 50. For any expanded bunch $H$ of $\mathcal{A}_{\Sigma_{0}}^{\text {sys }}, \mathcal{K}_{H} \models \Sigma_{0}$

PROOF. Immediate consequence of Lemma 49.

Now, we prove the refutational completeness of the tableau method.

Theorem 51 (Refutational Completeness). For any set of state formulae $\Sigma_{0}$, if UnSat $\left(\Sigma_{0}\right)$ then there exists a closed tableau for $\Sigma_{0}$.

Proof. Suppose the contrary, that there exists no closed tableau for $\Sigma_{0}$. Then the systematic tableau $\mathcal{A}_{\Sigma_{0}}^{\text {sys }}$ would be open and there would be at least one expanded bunch $H$ in $\mathcal{A}_{\Sigma_{0}}^{\text {sys }}$. By Corollary $50, \mathcal{K}_{B} \mid=\Sigma_{0}$. Consequently $\Sigma_{0}$ would be satisfiable.

\subsection{Termination}

Most tableau systems for modal and temporal logics, satisfy the analytic super-formula property (ASP): for every finite set of formulae $\Sigma$, there exists a finite set that contains all the formulae that may occur in any tableau for $\Sigma$. Such a set is usually called the closure of $\Sigma$. The ASP also ensures the non-existence of infinite branches where all the nodes have different labels. Hence, by controlling loops, the finiteness of proof search can be ensured. In our case, as a consequence of the $\beta^{+}$-rules, the tableau system fails to satisfy the ASP, but it satisfies a slightly weaker variant which ensures completeness and that we call the weak analytic superformula property (WASP): for every finite set of state formulae $\Sigma_{0}$ there exists a finite set (usually called the local closure of $\Sigma$ ) that contains all the formulae that may occur in any (systematic) tableau for $\Sigma$ constructed by Algorithm $\mathcal{A}^{\text {sys }}$. For this purpose, the eventuality selection policy used in the $\mathcal{A}^{\text {sys }}$ is crucial.

Theorem 52 (Termination of the Tableau Method). For any set of state formulae $\Sigma_{0}$, the construction of the ex815 panded tableau $\mathcal{A}_{\Sigma_{0}}^{\text {sys }}$ terminates.

Proof. Tableau rules produce a finite branching, hence König's Lemma, applies. The subsumption-based simplification rules (Subsection 3.5) do prevent the generation of formulae containing the original eventuality when a "new variant" has been generated. By Propositions 44 and 45 , the application of a $\beta^{+}$-rule to a selected formula stops after a finite number of steps. Finally, Proposition 48 ensures that any open branch is eventually-covered.

Theorem 53 (Completeness of the Tableau Method). For any set of state formulae $\Sigma_{0}$, if $\Sigma_{0}$ is satisfiable then there exists a (finite) open expanded tableau for $\Sigma_{0}$.

PROOF. The existence of the systematic tableau $\mathcal{A}_{\Sigma_{0}}^{\text {sys }}$ suffices to prove this fact, by Theorem 52

\section{Conclusion}

We introduced a new logic, ECTL\#, in the family of BTL and its tree-style one pass tableau. This extends the expressiveness of fairness by a new class of fairness constraints with the $\mathcal{U}$ operator. The tableau method handles inputs in an 'analytic" way, due to the new, crucial for branching structures, concept of 'inner context', in which eventualities are to be fulfilled. The tableau rules that invoke the inner context, are essential to handle A-disjunctive formulae. Our analysis of A-disjunctive and E-conjunctive formulae and of the prioritisation of eventualities, based on their structure and the context for their fulfillment, are important from the methodological point of view. 
Our tableau technique is not directly extensible to $C T L^{\star}$. Without any significant modifications, $\beta^{+}$-rules become unsound for inputs that are beyond ECTL\# syntax due to nested path subformulae as in $\mathrm{A} \diamond(\circ p \wedge \mathrm{E} \circ \neg p)$ mentioned in Figure 1. However, for the proof of correctness of $\beta^{+}$-rules, we developed the technique to identify relevant stateformulae inside the specific path-modalities. This technique will be useful in studying more expressive logics (e.g. $\left(\mathrm{CL}^{\star}\right)$, as it allows to identify those subformulae that do not affect the 'context', thus enabling the simplification of he structures.

We note that the size of the systematic tableau for the input of size $m$ is bounded by $2^{2^{O\left(m^{2}\right)}}$ (see technical report at http://www.sc.ehu.es/jiwlucap/TechReport18.pdf). However, the method aims at the 'shortest' way to fulfil the eventualities and, for many examples, finds the first open bunch, giving us a model for the tableau input. This significantly reduces the complexity. Finally, the presented technique is amenable for implementation and this will be another stream of our future work. In the refinement and implementation of the algorithm we will be able to rely on similar techniques used in the implementation of its linear-time analogue.

\section{References}

[1] E. A. Emerson, J. Y. Halpern, Decision procedures and expressiveness in the temporal logic of branching time, Journal of Computer and System Sciences 30 (1) (1985) 1 - 24 (1985).

845 [2] E. A. Emerson, J. Y. Halpern, Sometimes and not never revisited: On branching versus linear time temporal logic, J. ACM 33 (1) (1986) 151-178 (1986).

[3] E. M. Clarke, E. A. Emerson, A. P. Sistla, Automatic verification of finite-state concurrent systems using temporal logic specifications, ACM Trans. Program. Lang. Syst. 8 (2) (1986) 244-263 (1986).

[4] B. Josko, Model checking of ctl formulae under liveness assumptions, in: T. Ottmann (Ed.), Automata, Languages and Programming, 14th International Colloquium, Springer-Verlag Berlin Heidelberg, Karlsruhe, Federal Republic of Germany, 1987, pp. 5-24 (1987).

[5] E. A. Emerson, C.-L. Lei, Temporal reasoning under generalized fairness constraints, in: M. B., V.-N. G. (Eds.), STACS 1986, Lecture Notes in Computer Science, vol 210, Springer-Verlag Berlin Heidelberg, Karlsruhe, Federal Republic of Germany, 1986, pp. 21-37 (1986).

[6] T. Berg, H. Raffelt, Model checking, in: M. Broy, B. Jonsson, J.-P. Katoen, M. Leucker, A. Pretschner (Eds.), Model-Based Testing of Reactive Systems, Springer-Verlag, Berlin Heidelberg, 2005, pp. 557-603 (2005).

[7] J. Sun, Y. Liu, J. S. Dong, H. H. Wang, Specifying and verifying event-based fairness enhanced systems, in: S. Liu, T. Maibaum, K. Araki (Eds.), Formal Methods and Software Engineering: 10th International Conference on Formal Engineering Methods ICFEM 2008, Springer Science and Business Media, Kitakyushu-City, Japan, 2008, pp. 5-24 (2008).

[8] J. Kretinsky, R. Ledesma Garza, Rabinizer 2: Small deterministic automata for LTL \GU, in: Automated Technology for Verification and Analysis - 11th International Symposium, ATVA 2013, Springer, Heidelberg Dordrecht London New York, 2013, pp. 446-450 (2013).

[9] E. A. Emerson, Temporal and modal logic, in: J. van Leeuwen (Ed.), Handbook of Theoretical Computer Science (Vol. B), MIT Press, Cambridge, USA, 1990, pp. 995-1072 (1990).

[10] N. Markey, Temporal logics course notes, Master Parisien de Recherche en Informatique, Paris, France (2013). URL http://www.lsv.ens-cachan.fr/Publis/PAPERS/PDF/NM-coursTL13.pdf

[11] K. Brünnler, M. Lange, Cut-free sequent systems for temporal logic, Journal of Logic and Algebraic Programming 76 (2) (2008) 216-225 (2008).

[12] J. Gaintzarain, M. Hermo, P. Lucio, M. Navarro, F. Orejas, Dual systems of tableaux and sequents for PLTL, Journal of Logic and Algebraic Programming 78 (8) (2009) 701-722 (2009).

[13] P. Abate, R. Goré, F. Widmann, One-pass tableaux for computation tree logic, in: N. Dershowitz, A. Voronkov (Eds.), Logic for Programming, Artificial Intelligence, and Reasoning, Springer Berlin Heidelberg, Berlin, Heidelberg, 2007, pp. 32-46 (2007).

870 [14] O. Friedmann, M. Latte, M. Lange, A decision procedure for CTL* based on tableaux and automata, in: J. Giesl, R. Hähnle (Eds.), Automated Reasoning, Springer Berlin Heidelberg, Berlin, Heidelberg, 2010, pp. 331-345 (2010).

[15] A. Pnueli, Y. Kesten, A deductive proof system for CTL*, in: L. Brim, M. Křetínský, A. Kučera, P. Jančar (Eds.), CONCUR 2002 Concurrency Theory, Springer Berlin Heidelberg, Berlin, Heidelberg, 2002, pp. 24-40 (2002).

[16] J. C. MaCabe-Dansted, M. Reynolds, Rewrite rules for CTL*, Journal of Applied Logic 21 (2017) 24 - 56 (2017).

875 [17] M. Reynolds, A tableau for CTL ${ }^{\star}$, in: A. Cavalcanti, D. Dams (Eds.), FM 2009: Formal Methods, Second World Congress, Eindhoven, The Netherlands, November 2-6, 2009. Proceedings, Vol. 5850 of Lecture Notes in Computer Science, Springer, 2009, pp. 403-418 (2009).

[18] M. Reynolds, A tableau-based decision procedure for CTL*, Formal Aspects of Computing 23 (6) (2011) 739-779 (2011).

[19] S. Cerrito, A. David, V. Goranko, Optimal tableau method for constructive satisfiability testing and model synthesis in the alternating-time temporal logic ATL+, Vol. 17, 2014 (2014).

[20] R. Gore, Tableau methods for modal and temporal logics, in: M. D’Agostino, D. M. Dov Gabbay, R. Hähnle, J. Posegga (Eds.), Handbook of Tableau Methods, Springer, Netherlands, Dordrecht, 1999, pp. 297-396 (1999).

[21] A. Bolotov, M. Hermo, P. Lucio, Extending Fairness Expressibility of ECTL+: A Tree-Style One-Pass Tableau Approach, in: N. Alechina, K. Nørvåg, W. Penczek (Eds.), 25th International Symposium on Temporal Representation and Reasoning (TIME 2018), Vol. 120 of Leibniz International Proceedings in Informatics (LIPIcs), Schloss Dagstuhl-Leibniz-Zentrum fuer Informatik, 2018, pp. 5:1-5:22 (2018).

[22] R. S. Streett, E. A. Emerson, The propositional mu-calculus is elementary, in: J. Paredaens (Ed.), Automata, Languages and Programming, 11th Colloquium, Antwerp, Belgium, July 16-20, 1984, Proceedings, Vol. 172 of Lecture Notes in Computer Science, Springer, 1984, pp. 465-472 (1984).

[23] O. Kupferman, M. Y. Vardi, P. Wolper, An automata-theoretic approach to branching-time model checking, J. ACM 47 (2) (2000) $312-360$ (2000). 
Click here to access/download Source files (.tex, .doc, .docx, .eps, etc.) bibtemporal.bib 
Click here to access/download Source files (.tex, .doc, .docx, .eps, etc.) Closed-Tableau.JPG 
Click here to access/download Source files (.tex, .doc, .docx, .eps, etc.) commands-ECTLsharp.tex 
Click here to access/download Source files (.tex, .doc, .docx, .eps, etc.) ectlsharp-tcs.tex 
Click here to access/download Source files (.tex, .doc, .docx, .eps, etc.) ElementaryLeaves.pdf 
Click here to access/download Source files (.tex, .doc, .docx, .eps, etc.) Open-Bunch.JPG 